\title{
Evaluation of Ultrasonic Velocity Tests to Characterize Extraterrestrial Rock Masses
}

Thomas W. Edge II

Follow this and additional works at: https://researchrepository.wvu.edu/etd

\section{Recommended Citation}

Edge II, Thomas W., "Evaluation of Ultrasonic Velocity Tests to Characterize Extraterrestrial Rock Masses" (2017). Graduate Theses, Dissertations, and Problem Reports. 5528.

https://researchrepository.wvu.edu/etd/5528

This Thesis is protected by copyright and/or related rights. It has been brought to you by the The Research Repository @ WVU with permission from the rights-holder(s). You are free to use this Thesis in any way that is permitted by the copyright and related rights legislation that applies to your use. For other uses you must obtain permission from the rights-holder(s) directly, unless additional rights are indicated by a Creative Commons license in the record and/ or on the work itself. This Thesis has been accepted for inclusion in WVU Graduate Theses, Dissertations, and Problem Reports collection by an authorized administrator of The Research Repository @ WVU. For more information, please contact researchrepository@mail.wvu.edu. 


\title{
Evaluation of Ultrasonic Velocity Tests to Characterize Extraterrestrial Rock Masses
}

\author{
Thomas W. Edge II \\ Thesis submitted \\ to the Statler College of Engineering and Mineral Resources \\ at West Virginia University
}

in partial fulfillment of the requirements for the degree of

Master of Science in

Mining Engineering

\author{
Aaron Noble, Ph.D., Chair \\ Brijes Mishra, Ph.D. \\ Thomas Evans, Ph.D. \\ Department of Mining Engineering \\ Morgantown, West Virginia \\ 2017
}

Keywords: Nondestructive Tests, Rock Mechanics, Ultrasonic Pulse Velocity, Asteroid Capture, Lunar and Martian Regolith, In-Situ Resource Utilization

Copyright 2017 Wesley Edge 


\section{Abstract \\ Evaluation of Ultrasonic Velocity Tests to Characterize Extraterrestrial Rock Masses \\ Thomas Wesley Edge II}

NASA and other private companies are currently developing mission concepts to explore and extract resources from various extraterrestrial resources, including asteroids, lunar ice, and Martian regolith. Unfortunately, information on the geotechnical and mechanical properties of these potential resources is extremely sparse and often based on limited test data from surrogate samples (e.g. meteorites). Since Earthbased observational measurements alone cannot quantify these critical geotechnical properties, target specimens must be tested in-situ using non-destructive techniques. This data will mitigate risks caused by uncertainty in material properties. While numerous nondestructive rock characterization techniques are common for terrestrial applications, these methods must be adapted to account for physical properties and environmental conditions of space.

This thesis investigates the use of ultrasonic pulse velocity (UPV) tests for extraterrestrial rock characterization by evaluating the effects of physical sample properties on the signal attenuation of ultrasonic waves. An initial review of standard laboratory UPV testing methods and expected space conditions isolated four key disparities between the two testing environments: (1) sample dimensions, (2) regolith cover, (3) sample surface roughness, and (4) utility of a gel couplant. Laboratory tests were then performed to assess the influence of these parameters in simulated space conditions, using concrete beams to simulate large sample sizes, and ground limestone to simulate regolith cover. The ensuing data analysis identified strategies to ensure adequate signal transmission and data interpretation.

Overall, the experimental results show that the signal attenuation curves can be constructed and simple regression curves can provide estimations of the "true" $\mathrm{P}$ wave velocity even without a gel couplant. For the case of large sample sizes, simple exponential regression of the measured $\mathrm{P}$-wave velocity at various transmission lengths can be extrapolated to zero length to estimate the true value. For the case of regolith cover, the particle top size and particle size distribution affect signal attenuation; however, an exponential fit to measured velocity versus depth data can be used to accurately predict true sample velocity at zero depth. Additionally, testing with rough samples showed that compacted regolith can be used to ensure contact between the sample and the UPV transmitter, and this method produced similar attenuation curves to those determined from smooth samples. Altogether, these data indicate the reliability of UPV to characterize material in-situ; however, without a gel couplant, UPV measurements become increasingly imprecise. 


\section{Acknowledgments}

My sincere gratitude goes to my advisor and committee chair, Dr. Aaron Noble. Dr. Noble is an exemplary professor whose passion for seeking greater knowledge is seconded only by his unwavering dedication to his students. I am grateful for the guidance and inspiration that he has given me. Thank you, Dr. Noble.

I would like to thank my committee members Dr. Thomas Evans and Dr. Brijes Mishra for imparting their expert advice and wisdom to me. I will never forget the lessons you taught me.

Without the support of my parents, Mark and Deborah, and my siblings, Keenan and Joan, the completion of this thesis would not have been possible. Thank you for giving me the motivation to strive for excellence.

Additionally, I would like to thank my peers at West Virginia University and to my lifelong friends who have shared their thoughts, criticisms, and support throughout my graduate studies.

I would like to acknowledge the NASA Space Technology Research Grants Program for funding the efforts of this thesis. This work was supported by an Early Stage Innovations grant from NASA's Space Technology Research Grants Program. 


\section{Contents}

1 Introduction $\quad 1$

1.1 Background .......................... . . 1

1.1.1 Asteroid Redirect Mission Overview . . . . . . . . . . . 2

1.1.2 Lunar and Martian ISRU Overview . . . . . . . . . . . . 3

1.1.3 Introduction to Non-Destructive Testing . . . . . . . . . 4

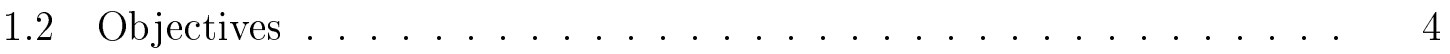

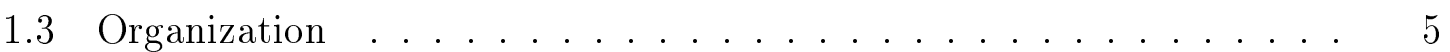

2 Literature Review $\quad 7$

2.1 Extraterrestrial Rock Masses . . . . . . . . . . . . 7

2.1.1 Background .................... 7

2.1.2 Asteroid Information . . . . . . . . . . . . . . 7

2.1.3 Martian and lunar information . . . . . . . . . . . 10

2.1.4 Summarized Information _. . . . . . . . . . . . . 14

2.2 UPV Rock Characterization Technique . . . . . . . . . . 18

2.2.1 UPV Background . . . . . . . . . . . . . 18

2.2.2 Waveform Overview . . . . . . . . . . . 18

2.2.3 Data Acquisition . . . . . . . . . . . . . . 19

2.2.4 Data Utilization ................ 20

2.2.5 Environmental and In-Situ Testing Issues . . . . . . . . . . . 24

2.2 .6 Summary . . . . . . . . . . . . . . 27

3 Materials and Methods $\quad 29$

3.1 Experimental Objectives . . . . . . . . . . . . . . . 29

3.2 Sample Procurement . . . . . . . . . . . . . . . . 29

3.2.1 Rock Samples . . . . . . . . . . . . . . 29

3.2 .2 Concrete Samples . . . . . . . . . . . . . 30

3.2.3 Regolith Generation .............. 32

3.3 Methods $/$ Procedures . . . . . . . . . . . . . . . 33

3.3.1 Standard Test Protocols for UPV . . . . . . . . . . . 33 
$3.3 .2 \quad$ Data Processing . . . . . . . . . . . . . . . . . . 33

3.3 .3 Length Testing . . . . . . . . . . . . . . . . 33

3.3 .4 Regolith Testing . . . . . . . . . . . . . . . 33

3.3 .5 Roughness Testing . . . . . . . . . . . . . 36

3.3 .6 Ice Testing . . . . . . . . . . . . . . . . . . 38

3.4 Analytical Methods . . . . . . . . . . . . . . . . 40

3.4 .1 Length Analysis . . . . . . . . . . . . . . . . 40

3.4.2 Regolith and Roughness Analysis . . . . . . . . . . 40

4 Results and Discussion $\quad 42$

4.1 Length of Sample . . . . . . . . . . . . . . . . . . . 42

4.1 .1 Direct and Semi-Direct Testing . . . . . . . . . . . 42

4.1 .2 Error Analysis . . . . . . . . . . . . . . . 43

4.2 Regolith Testing . . . . . . . . . . . . . . . . . 45

4.2 .1 Statistical Analysis of R1 . . . . . . . . . . . 45

4.2.2 Procedure Revisions and R2 Statistical Analysis . . . . . . . 49

4.2 .3 Isolating the Influence of Particle Size . . . . . . . . . . 52

4.3 Surface Roughness . . . . . . . . . . . . . . . . 57

4.3.1 Isolating the Parameter . . . . . . . . . . . 57

4.3.2 Addition of Surface Regolith and Comparison to R4 . . . . . 58

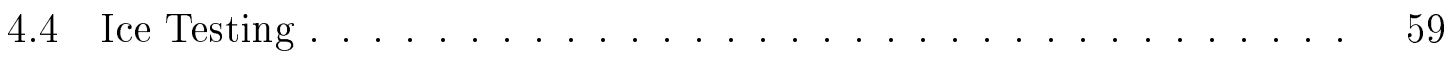

4.4 .1 Variance in Ice Concentration . . . . . . . . . . . . . 59

4.4 .2 Distribution of Regolith Sizes and Ice . . . . . . . . . 60

4.5 Discussion and Implications $\ldots \ldots \ldots \ldots \ldots$

4.5 .1 Sample Length . . . . . . . . . . . . . . . 62

4.5 .2 Regolith Cover . . . . . . . . . . . . . . . . 62

4.5.3 Sample Roughness . . . . . . . . . . . . . . . . 63

4.5.4 Gel Couplant . . . . . . . . . . . . . . . . . 63

5 Conclusions and Recommendations $\quad 64$

5.1 Conclusions . . . . . . . . . . . . . . . . 64

5.2 Recommendations/Future Work . . . . . . . . . . . 66 


\section{List of Figures}

1.1 Artist's Interpretation of Asteroid Redirect Vehicle (NASA, 2016) . . 2

2.1 Different Regions of the Moon . . . . . . . . . . . . . . 12

2.2 Particle Assemblies . . . . . . . . . . . . . . . . 13

2.3 Transducer Cross-Section . . . . . . . . . . . . . . . . 19

2.4 Example of UPV Structure . . . . . . . . . . . . . . . 20

2.5 Void Space Example . . . . . . . . . . . . . . 22

3.1 Limestone (L) and Chalk (R) Cores . . . . . . . . . . . . 30

3.2 Concrete Beams Immediately After Pour. From Left to Right: 6 m, 3 $\mathrm{m}$, and $1 \mathrm{~m}$ beams . . . . . . . . . . . . . 31

3.3 Regolith Generation Equipment . . . . . . . . . . . . . . . . . . 32

3.4 Regolith Size Classes . . . . . . . . . . . . . . . . 32

3.5 Methods of Transmission . . . . . . . . . . . . . 33

3.6 Limestone Core . . . . . . . . . . . . . . . . . . 34

3.7 Limestone Sample with Groove Cut (L) and Limestone Sample within PLT-10 (R) . . . . . . . . . . . . . . 37

3.8 Before and After Homogenization . . . . . . . . . . . . 38

3.9 PVC Cast . . . . . . . . . . . . . . . . . . 39

3.10 Experiment Setup . . . . . . . . . . . . . . 39

4.1 Semi-Direct Testing of 6 meter Beam Compared to Direct Testing of 1 ,3, and 6 meter Beam . . . . . . . . . . . . . 43

4.2 Maximum Error of Corrected Values from Baseline Velocity With Gel Couplant (L) and Without Couplant (R) . . . . . . . . . 44

4.3 Box-Plot of All Corrected Values With Gel Couplant (L) and Without Gel Couplant $(\mathrm{R})$... . . . . . . . . . . . . 44

4.4 First Regolith Series Velocity Prediction Model . . . . . . . . . . 47

4.5 First Regolith Series Amplitude Prediction Model . . . . . . . . . . . 48

4.6 Second Regolith Series Velocity Prediction Model . . . . . . . . . . 51

4.7 Second Regolith Series Amplitude Prediction Model . . . . . . . . . . 51 
4.8 Regolith Depth vs. Longitudinal Wave Velocity Sorted by Particle Top Size (L) and Size Distribution (R) . . . . . . . . . . . . 52

4.9 Regolith Depth vs. Longitudinal Wave Velocity Across All Size Classes 53

4.10 Corrected Wave Velocity for Each Size Classes . . . . . . . . . . 53

4.11 Regolith Depth vs. Longitudinal Wave Velocity Across Three Size Classes 55

4.12 Linearized Regolith Depth vs. Longitudinal Wave Velocity Across Three Size Classes . . . . . . . . . . . . . . . 55

4.13 Regolith Depth vs. Longitudinal Wave Velocity for Dissimilar Regolith

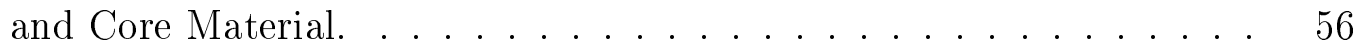

4.14 R5 Linearized Curves . . . . . . . . . . . . . . . . . 57

4.15 Comparison Between Smooth and Rough Samples With and Without Gel Couplant . . . . . . . . . . . . . . . . . . 58

4.16 Regolith Depth vs. Longitudinal Wave Velocity for Smooth and Rough Samples . . . . . . . . . . . . . . . . . 59

4.17 Regolith UPV Testing with a Monosized Regolith Mixed with Ice . . 60

4.18 Regolith Distribution and Ice Testing $\ldots \ldots \ldots$. . . . . . 60 


\section{List of Tables}

2.1 Asteroid Mineralogy and Expected Resources of the Three Main Asteroid Categories (After DeMeo et al. 2015; Nelson et al. 1993; Mazanek et al. 2015) . . . . . . . . . . . . . . . . 8

2.2 Composition of Regolith Samples (\% Wt) Taken from Mudstone at Yellowknife Bay, Gale Crater, Mars (After Vaniman et al., 2014) . . . 11

2.3 Mineralogical Compositions of Lunar Regolith Samples in \% Vol. (After Papike et al. 1991) _. . . . . . . . . . . . . . 13

2.4 Best Averages for Lunar Regolith Bulk Density, Relative Density, and Porosity (After Houston et al. 1974, Heiken et al. 1991) . . . . . . . . 14

2.5 Spacecraft and Vehicles with Mineralogical Determination Capabilities (After Ehlmann and Edwards 2014) . . . . . . . . . . . . . 16

2.6 Lunar Missions with Samples Returned . . . . . . . . . . . . 17

2.7 Equations Relating P-wave Velocity to UCS . . . . . . . . . . . 23

2.8 Normal Laboratory Testing Conditions (After Aydin, 2015; ASTM Standard D2845-08) . . . . . . . . . . . . . 24

2.9 UPV In-Situ Testing Conditions . . . . . . . . . . . . . 28

3.1 Baseline Data for Chalk and Limestone (After Savage, 2016) . . . . 30

3.2 Concrete Baseline Values . . . . . . . . . . . . . . 31

3.3 Parameters for Series 1 of Regolith Testing . . . . . . . . . . . . 35

3.4 Parameters for Series 2 of Regolith Testing . . . . . . . . . . . 35

3.5 Summary Test Matrix _................. 41

4.1 First Regolith Testing Series Results . . . . . . . . . . . . . 46

4.2 First Regolith Series Velocity Statistical Analysis . . . . . . . . . . 47

4.3 First Regolith Series Amplitude Statistical Analysis . . . . . . . . . . 48

4.4 Second Regolith Testing Series Results . . . . . . . . . . . . . . 50

4.5 Second Regolith Series Velocity Statistical Analysis . . . . . . . . . 51

4.6 Second Regolith Series Amplitude Analysis . . . . . . . . . . . . . 51

4.7 Comparison Between Trendlines . . . . . . . . . . . . 55

4.8 R5 Comparison Between Trendlines . . . . . . . . . . . 57 


\section{Chapter 1}

\section{Introduction}

\subsection{Background}

Over the next 20 years, missions to extraterrestrial bodies are increasing in importance for governments and private institutions to take place. These mission destinations include Near-Earth Asteroids (NEAs), the Moon, Mars, and other planetary bodies. The data collected from these missions will assist in planning long-term crewed missions to extraterrestrial bodies. One current initiative is the Asteroid Redirect Mission (ARM). This mission will attempt to intercept a large asteroid with an unmanned vehicle and return a boulder from its surface to a retrograde lunar orbit. A manned expedition will examine the boulder and return with samples to the Earth. Other long-term crewed missions to the Moon and Mars will depend on In-Situ Resource Utilization (ISRU) technologies that can extract and process resources found on these bodies to provide the goods and services for a crew. The success of ARM and ISRU will depend on direct measurements of the mechanical properties of the bodies; however, remote sensing is the primary means of determining these properties.

Terrestrially, extensive exploration and prospecting of possible mine sites is required before any mining takes place. Identifying and characterizing the ore body is necessary to to continue past these phases, which means that direct rock testing techniques already exist. These conventional rock testing techniques and the associated equipment can be adapted to operate within the environmental conditions on extraterrestrial bodies to determine the mechanical properties of rock masses. Over next three sections, further explanation of the ARM and ISRU efforts as well as the need for direct testing techniques will be provided. 


\subsubsection{Asteroid Redirect Mission Overview}

The ARM will attempt to capture a boulder off of a larger asteroid and transport the boulder to distant retrograde orbit around the Moon. The three segments of this mission are: Identification, the Asteroid Robotic Redirect Mission (ARRM) and the Asteroid Crewed Redirect Mission (ARCM). The identification segment will identify and characterize candidate Near-Earth Asteroids (NEAs) using telescopes, radar facilities, and space-based assets such as NASA's Spitzer Infrared Space Telescope and Near-Earth Object Wide-field Infrared Survey Explorer (NEOWISE) Space Telescope. These are the same assets that are being used to help catalog NEAs and identify those that can potentially impact the Earth (Mazanek et al., 2015).

The second segment, named the Asteroid Redirect Robotic Mission (ARRM), will robotically capture a boulder $2-4$ meters in diameter from a large NEA. The current design of the Asteroid Redirect Vehicle (ARV) allows the collection of a boulder with a mass up to 70 metric tons. Large NEAs with Earth-like orbits are preferred for this mission because they are less difficult to identify and characterize than smaller NEAs and they have a greater likelihood of having a boulder candidate that has favorable parameters for return transit to lunar orbit; however larger NEAs are are less plentiful than smaller NEAs (Mazanek et al., 2015). An example of the ARV design can be seen in Figure 1.1.

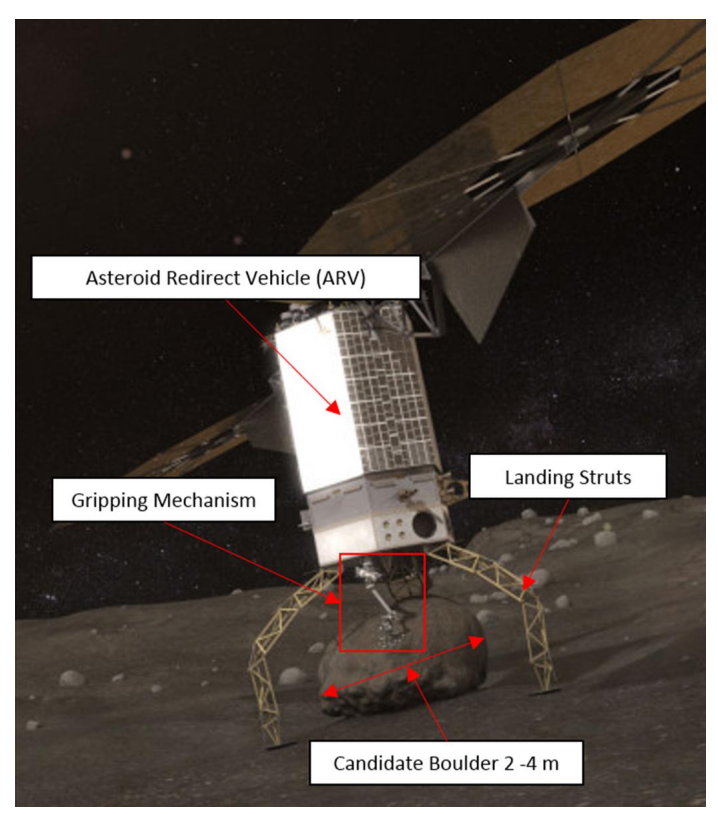

Figure 1.1: Artist's Interpretation of Asteroid Redirect Vehicle (NASA, 2016)

The final segment of the ARM, named the Asteroid Redirect Crewed Mission (ARCM), will send a team of astronauts aboard NASA's Orion spacecraft and the 
Space Launch System (SLS) to rendezvous with the returned boulder and conduct Extravehicular Activities (EVAs) to collect and return the samples to Earth for analysis. Both the ARRM and ARCM are planned for the mid-2020s.

Obtaining in-situ samples are critical to technology development because these sample have not undergone significant chemical and physical changes like a meteorite has when it passes through the Earth's atmosphere. These samples will allow further developments in resource extraction technology, which provides incentives for government and private enterprises. The mission also has objectives of demonstrating planetary defense since the spacecraft will be able to redirect an extraterrestrial body onto a new course (Mazanek et al., 2015).

During the second mission segment, the mechanical properties and internal structure of the boulder candidates will determine which one will be selected for return to lunar orbit. The candidate has to have a stable and cohesive structure in order to survive the return flight. To determine these properties, the ARV has to have a suite of geotechnical characterization tools. Ideally, these tools must be lightweight and small in order to save space and mass for other mission critical items. Space on board the vehicle reserved for rock characterization has to contain robust tools with validated procedures that are able to gather precise information for comparisons between candidates. These rock characterization technologies have to be developed independently or converted from conventional techniques already in use with the latter being remarkably easier to test and evaluate first.

\subsubsection{Lunar and Martian ISRU Overview}

In-Situ Resource Utilization is the creation of goods and services from processed resources on an extraterrestrial body. The products from these processes are supposed to alleviate the payload on missions to extraterrestrial bodies since they can be created from the material on site. The available space can be allocated towards mission critical items on board the spacecraft since the consumables can be made insitu. Current ISRU technologies focus on the collection of water, which can be used for the creation of fuel propellant or for life support, both of which are critical for long-term crewed missions to the Moon and Mars. Other valuable resources include metal-oxide minerals such as ilmenite or hematite for manufacturing or volatiles and organic compounds for additional life-support measures (Anand et al., 2012). Although determining chemical composition is necessary to finding valuable minerals or substances, these tests cannot provide information on physical and mechanical properties like density, porosity, cohesion, and strength, which are critical for extraction and processing. Currently, no equipment is available that can precisely characterize mechanical properties in-situ. The importance of testing rock masses in-situ is to 
characterize material under their natural environmental and physical conditions instead of the conditions within a laboratory. Testing in-situ will also provide data in real-time instead of waiting for results after testing in a lab.

\subsubsection{Introduction to Non-Destructive Testing}

Recent missions to NEA's and NASA's Martian rovers have shown the need for further characterization efforts. Non-Destructive Tests (NDTs) can be converted to support the extraterrestrial rock characterization efforts. Although NDTs are used conventionally for testing steel, concrete, and rock quality, the conditions encountered on extraterrestrial bodies are significantly different from those on Earth. These testing conditions are also compounded by the fact that terrestrial uses of NDTs are dictated by correlations from NDT responses to mechanical rock properties specific to an individual rock mass, e.g. one correlation between an NDT response to a mechanical property will not apply to two rock masses even if they are the same rock type. The general relationships that exist between NDT responses and mechanical properties are still able to classify material into broad categories (e.g. weak, average, strong, etc.). Ultimately, investigations into how material and environmental properties influence NDT responses will allow for the modifcation of these tools to be suited specifically for use on materials within a space environment.

Despite numerous NDTs, the focus of this body of research was limited to one technique: ultrasonic pulse velocity (UPV). UPV uses ultrasonic waves to characterize the elastic properties of a specimen as well as its internal structure. The velocity an ultrasonic wave travels at is dictated by a material's mechanical properties; however, testing with UPV is typically conducted with ideal samples and within a laboratory. These conditions are not present when testing in-situ, so in order to apply UPV for extraterrestrial rock characterization, its applicability for in-situ testing must be assessed.

\subsection{Objectives}

Given the opportunity previously mentioned, a research project was initiated to test the application of UPV as part of the suite of rock characterization techniques on-board several spacecraft and rovers.

The objectives for this body of research are to:

- Identify and assess the impacts of in-situ physical sample properties that may affect UPV signal attenuation.

- Develop procedures for mitigating the effects of the most influential properties. 
- Assess the ability of UPV to characterize changes in ice content.

The identification of physical rock properties was conducted through investigation of standard testing protocols and literature review. Then, a rigorous testing regiment assessed the effects on ultrasonic waves by these properties. Finally, an analysis of the results used to develop procedures to overcome the influence of these properties and provide a method to estimate the true wave velocity of the material.

Given UPV's ability to monitor changes in mechanical properties, a secondary objective is assessing the feasibility of using UPV to measure changes in ice content. Ice is a valuable material for ISRU efforts and identifying its location and its concentration will help create long-term missions to extraterrestrial bodies.

Previous works from the same research group have evaluated the influence of subzero temperatures on UPV responses and robotic implementation of UPV (Savage, 2016; Goodman, 2016). Savage (2016) evaluated the effects of the extreme freezing temperatures on UPV responses and elastic moduli across three rock types. Goodman (2016) analyzed effects from transducer force, transducer alignment, transducer type, transducer angular offset, and specimen length on ultrasonic velocity in order to develop procedures for robotic implementation of UPV. The research within this research differentiates itself from past work by restricting itself to evaluating the influence of physical sample properties on UPV responses.

\subsection{Organization}

This thesis is organized into five chapters. Chapter 1 includes a description of background information and the motivation for this project. The information provides an overview of previous and planned missions of extraterrestrial bodies as well as the proposed objectives to be completed in this thesis.

Chapter 2 is a literature review, which is comprised of asteroid classification and characterization as well as the mineralogical and geotechnical characteristics of the surfaces of the Moon and Mars. This is followed by an explanation of the need for NDT's and an overview of Ultrasonic Pulse Velocity testing. This overview includes information about the current uses of UPV and the conditions faced when testing in-situ during a mission to an extraterrestrial body.

Chapter 3 describes the materials and methods involved to test the influence of the unique testing conditions found in the previous chapter on longitudinal wave velocity and wave amplitude. The experimental design and procedure for each test series are discussed in full detail. The chapter also includes the methods used to analyze the data. 
Chapter 4 reviews the results from each test series and discuss the impacts each condition has on longitudinal wave velocity and wave amplitude.

Chapter 5 summarizes the key findings of this work and introduces opportunities for further research and development. 


\section{Chapter 2}

\section{Literature Review}

\subsection{Extraterrestrial Rock Masses}

\subsubsection{Background}

The success of missions to Mars, Lunar, and asteroid will depend on rock characterization to estimate the mechanical properties of rock masses and prioritize them according to the mission parameters. In-Situ Resource Utilization (ISRU) efforts depend on using specific minerals and substances to produce consumables that will enable long-term investments on extraterrestrial surfaces, but the properties of the rock masses will determine the rocks that are suitable for resource extraction. Asteroid exploration missions such as the proposed Asteroid Redirect Mission (ARM) depend on rock characterization to select a suitable target to bring back into lunar orbit for further study. These missions require basic information about the composition and structure of rock samples to maximize their effectiveness. The following sections provide explanations on asteroid classification as well as martian and lunar regolith mineralogical compositions in order to illustrate the need for in-situ rock characterization techniques

\subsubsection{Asteroid Information}

Most asteroids are thought to be the material that never accreted to form planets (Nelson et al., 1993). Asteroids are rich with minerals and compounds that will be used for human expansion into space; however, current classification and structural characterizations are accomplished by using remote sensing techniques or by laboratory analysis of meteorites (Williams, 2015). The following subsections explain

current asteroid classification and known internal structure characteristics as well as the need for direct for direct rock characterization techniques. 
Table 2.1: Asteroid Mineralogy and Expected Resources of the Three Main Asteroid Categories (After DeMeo et al. 2015; Nelson et al. 1993; Mazanek et al. 2015)

\begin{tabular}{|c|c|c|c|}
\hline $\begin{array}{l}\text { Asteroid } \\
\text { Class }\end{array}$ & Minerals & $\begin{array}{l}\text { Meteorite } \\
\text { Analogs }\end{array}$ & $\begin{array}{l}\text { Expected } \\
\text { Resources }\end{array}$ \\
\hline $\mathrm{C}$ & $\begin{array}{l}\text { olivine, pyroxene, } \\
\text { clays, organics }\end{array}$ & $\begin{array}{l}\text { Most carbonaceous } \\
\text { chondrites }\end{array}$ & $\begin{array}{l}\text { hydrocarbons, } \\
\text { volatiles, water, } \\
\text { metals }\end{array}$ \\
\hline $\mathrm{S}$ & $\begin{array}{l}\text { opaques, carbon, } \\
\text { phyllosilicates, } \\
\text { indications of } \\
\text { olivine and } \\
\text { pyroxene }\end{array}$ & $\begin{array}{l}\text { Some chondrites, } \\
\text { basaltic and } \\
\text { primitive achondrites }\end{array}$ & $\begin{array}{l}\text { oxides, oxygen, } \\
\text { metals }\end{array}$ \\
\hline $\mathrm{X}$ & $\begin{array}{l}\text { opaques, carbon, } \\
\text { low-Fe pyroxene, } \\
\text { enstatite, } \\
\text { oldhamite }\end{array}$ & $\begin{array}{l}\text { most carbonaceous } \\
\text { chondrites, Metal } \\
\text { rich chondrites, } \\
\text { enstatites, silicate } \\
\text { rich irons }\end{array}$ & $\begin{array}{l}\mathrm{Fe}, \mathrm{Ni}, \mathrm{Pt} \text { group } \\
\text { metals }\end{array}$ \\
\hline
\end{tabular}

\section{Classification (Mineralogy and Composition)}

Asteroids are classified based upon their spectral reflectance. Spectral reflectance is produced by different minerals on the surface of the asteroid absorbing light at different wavelengths (Tholen, 1989). There are eight categories that asteroids can be classified as: C-, S-, X-, A-, D-, T-, O-, R-, and V-categories with the C- (carbonaceous), S- (siliceous), and X- (varied) types being the predominant categories (DeMeo et al., 2015). Although remote spectroscopy is a common technique to classify asteroids, each category has to have a broad enough wavelength range to distinguish itself from another category. Most minerals on the surface of an asteroid do not produce distinguishable spectral features from other materials and spectral reflectance data is also affected by grain size, temperature, viewing geometry, and space weathering processes that cause changes on the surface over time (DeMeo et al., 2015).

The second common classification technique uses meteorite analogs. The three main types of asteroids based on meteorite analogs are: stony, stony iron, and iron. This classification technique has a major bias towards asteroids that contain materials that survive the intense heat and acceleration through the Earth's atmosphere and the subsequent impact (Nelson et al., 1993). Essentially, classification techniques cannot be used to precisely determine the compositions of asteroids; however, they can identify significant similarities and differences between groups of asteroids. Table 2.1 contains a summary of the dominant asteroid categories and common minerals within each category. 


\section{Internal Structure Characteristics}

The internal structure of an asteroid is difficult to characterize since most methods of rock characterization require direct measurements. Currently, no means of directly testing asteroids in situ exist; however, some simple mechanical properties can be approximated by using remote sensing as well as meteorite analogs. Depending on the physical properties and the collision history of the asteroid, its internal structure can be classified as one of two distinct categories: monolithic and rubble-pile (Benavidez et al., 2012). Monolithic asteroids are large rock bodies that have maintained their structural integrity by largely avoiding fracture and fragmentation events caused by catastrophic disruptions caused by collisions with another asteroid. Alternatively, rubble piles have been affected by these disruptions but rather than splitting into individual asteroids, the original parent body merely fragmented with the remains bound together by gravitational and cohesive forces (Scheeres et al., 2010; Wilkison et al., 2002).

From the reflectance spectrum and meteorite analogs, two physical properties of the asteroid can be calculated readily: bulk density and porosity. First, the mass of the asteroid is estimated by measuring the gravitational impact on surrounding bodies or spacecraft. Then the volume can be calculated by using infrared radiometry or imaging data from neighboring spacecraft to determine the dimensions of the asteroid (Britt et al., 1987; Carry, 2012). The porosity is calculated by using the estimated bulk density, the reflectance spectrum that provides a meteoritic compositional analog, and the grain density, and average porosity of the analog meteorite (Britt et al., 1987). At present, these are the only physical properties that can be estimated using remote sensing techniques and meteorite analogs.

\section{The Presence of Ice}

The presence of water on asteroids is critical to ISRU developments since water can be used to create rocket propellant or life support. Most of the data about water concentrations used to come from meteorite analogs that do not contain water but have alterations from reactions with water leaving the meteorite (Lewis and Hutson, 1993). Further developments in remote sensing techniques showed that depending on the distance away from the sun and the rotation of the asteroid, water concentration in asteroids can be as high as $25 \%$ by mass (Thomas et al., 2005). The correct maximum value for water concentration in an asteroid will be more precisely determined when the asteroids can be directly tested, but even through indirect means of determining water concentrations, asteroids are shown as promising resources for ISRU efforts. 


\subsubsection{Martian and lunar information}

Considerable efforts for ISRU technology development are allocated towards Mars and the Moon since they are likely destinations for future crewed missions. The subsequent sections discuss the mineralogical and geotechnical properties of the bodies as well as the primary data collection methods.

\section{Mineralogy, Composition, and Concentrations of Ice of Mars}

Although the mineralogical composition of Mars will likely never be mapped in its entirety, readily identifiable concentrations of valuable minerals will be useful for initial ISRU efforts. So far, dozens of minerals have been identified with mafic minerals as the dominate mineral type. Due to ancient volcanic activities, the upper crust of Mars is broadly basaltic, but the abundance of other minerals vary based on location (Ehlmann and Edwards, 2014). On younger terrains, ferric oxides and silica bearing minerals are the predominant mineral types as indicated by spectroscopic images taken from the OMEGA satellite (Bibring et al., 2006). The surface regolith samples taken by the Mars Science Laboratory (MSL) on board the Curiosity rover also validate the data sets collected with olivine, pyroxene, and plagiocase making up a large portion of sample compositions (Vaniman et al., 2014). The data from three of these samples can be seen in Table 2.2. The amorphous minerals are particles that could not be properly identified by X-Ray Diffraction (XRD) and simply put into their own category.

Two mineral types that are of particular interest for ISRU development are phyllosilicates and sulfates because they indicate the presence of water. Other than the glaciers at the mid-latitudes, concentrations of these minerals are prime candidates for water resources for ISRU technologies (Abbud-Madrid et al., 2016). From the Table 2.2 , the phyllosilicate of interest is smectite and the sulfates of interest are anhydrite and bassanite. These minerals absorb water and lock the molecules into the mineral structure. Thus, extraction can be a heat source that produces enough energy to release the locked water molecules from the rest of the mineral (Abbud-Madrid et al., 2016). As an aside, anhydrite does not contain water, its presence suggests that its water bearing cousins are in the vicinity such as bassanite and gypsum. Although spectroscopic data does not provide information on the mechanical properties of a sample, data about regolith cohesion, friction angles, and qualitative assessments of grain sizes was collected from the Mars Exploration Rover (MER), Opportunity. The wheels on the rover dug trenches in the regolith and the changes in rover telemetry were based on the physical properties of the surface regolith (Sullivan et al., 2011). From the data collected, the regolith friction angles were $30^{\circ}-37^{\circ}$, which are consistent with dry, fine-grained sands. Regolith cohesion was estimated to be $0-2 \mathrm{kPa}$. 
Table 2.2: Composition of Regolith Samples (\% Wt) Taken from Mudstone at Yellowknife Bay, Gale Crater, Mars (After Vaniman et al., 2014)

\begin{tabular}{|lccc|}
\hline Mineral & Rocknest & John Klein & Cumberland \\
\hline Plagiocase & 29.8 & 22.4 & 22.2 \\
Fe-Forsterite & 16.4 & 2.8 & 0.9 \\
Augite & 10.7 & 3.8 & 4.1 \\
Pigeonite & 10.1 & 5.6 & 8.0 \\
Orthopyroxene & - & 3.0 & 4.1 \\
Magnetite & 1.5 & 3.8 & 4.4 \\
Anhydrite & 1.1 & 2.6 & 0.8 \\
Bassanite & - & 1.0 & 0.7 \\
Quartz & 1.0 & 0.4 & 0.1 \\
Sanidine & 0.9 & 1.2 & 1.6 \\
Hematite & 0.8 & 0.6 & 0.7 \\
Ilmenite & 0.7 & - & 0.5 \\
Akaganeite & - & 1.1 & 1.7 \\
Halite & - & 0.1 & 0.1 \\
Pyrite & - & 0.3 & - \\
Pyrrohite & - & 1.0 & 1.0 \\
Smectite & - & 22 & 18 \\
Amorphous & 27 & 28 & 31 \\
\hline
\end{tabular}

\section{Mineralogy, Composition, and Concentrations of Ice on the Moon}

The regolith on the surface of the moon is also of particular interest for ISRU development. The moon contains two distinct two regions that hold two different petrologies and lithologies: highlands and mare plains. The highlands were formed due to primordial volcanic processes that generated a silicate-rich magma ocean that spread over the moon. The maria formed later with a greater concentration of basaltic lava flows giving the maria their darker tone (Heiken et al., 1991). Examples of the two kind of regions can be seen in Figure 2.1.

The rocks in these regions contain distinct characteristics that indicate their formation. Basaltic rocks and glasses with high iron content are indicative of lunar mare whereas the highlands contain higher concentrations of plagiocase, magnesium, and rocks known as KREEPs, which are highly enriched in potassium (K), rare earth elements (REE), and phosphorus (P). The regolith formed within these regions also reflect these differences between formations(Heiken et al., 1991).

The primary mechanism that generated the surface regolith on the moon was the ancient bombardment of asteroids and comets. The impacts generated new particles, with some of them settling back on the surface or ejecting from the moon's gravitational pull. The impacts also crushed and mixed the existing regolith around the 


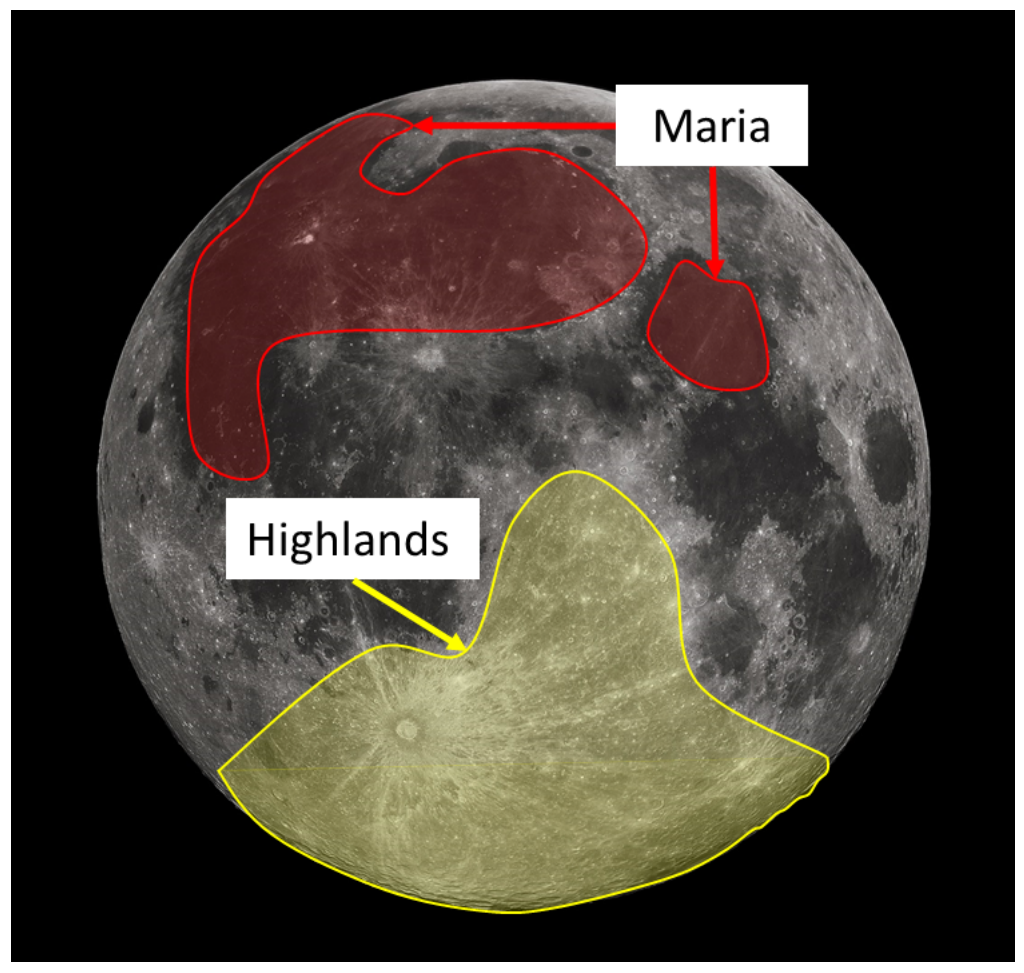

Figure 2.1: Different Regions of the Moon

moon. The intense heat from these impacts generated glasses and particles fused together called agglutinates. With a almost non-existent atmosphere to protect the lunar surface, solar and cosmic rays produced nuclear reactions between particles through the regolith. Due to these random and localized events, mineral concentrations in the regolith are not uniform over long distances (Heiken et al., 1991).

The differences in mineral concentrations in different regolith samples taken during select Apollo missions can seen Table 2.3. Of particular note are the differences between plagioclase, pyroxene and olivine between highland and mare samples.

Ilmenite $\left(\mathrm{FeTiO}_{3}\right)$ is of particular interest for ISRU technology because it can be reduced into three products: iron, titanium oxide, and oxygen. Not only can the iron and titanium be used for fabrication projects, but this process yields free oxygen, which can be used for fuel, water formation, and breathing air. Although reduction processes for different minerals yield similar products, ilmenite reduction is currently favored due to its simplicity (Anand et al., 2012).

The lunar regolith mechanical properties have been directly measured, unlike the martian regolith. Bulk density, relative density, and porosity as well as other properties have been measured for separate lunar samples. The relative density is a ratio between a sample's measured bulk density and data from other known samples, expressed as a percent. Due to how particles are organized, regolith density and porosity 
Table 2.3: Mineralogical Compositions of Lunar Regolith Samples in \% Vol. (After Papike et al. 1991)

\begin{tabular}{|lcccc|}
\hline Mineral & $\begin{array}{c}\text { A-16 } \\
\text { (Highland) }\end{array}$ & $\begin{array}{c}\text { A-17 } \\
\text { (Highland) }\end{array}$ & $\begin{array}{c}\text { A- 16 } \\
\text { (Mare) }\end{array}$ & $\begin{array}{c}\text { A-17 } \\
\text { (Mare) }\end{array}$ \\
\hline Plagioclase & 34.1 & 39.3 & 12.9 & 34.1 \\
Pyroxene & 38 & 27.7 & 61.1 & 30.1 \\
Olivine & 5.9 & 11.6 & 5.3 & 0.2 \\
Silica & 0.9 & 0.1 & - & - \\
Ilmenite & 0.4 & 3.7 & 0.8 & 12.8 \\
Mare Glass & 15.9 & 9.0 & 6.7 & 17.2 \\
Highland Glass & 4.8 & 8.5 & 10.9 & 4.7 \\
Others & - & - & 2.3 & 0.7 \\
Total & 100.0 & 99.9 & 100.0 & 99.8 \\
\hline
\end{tabular}

a)

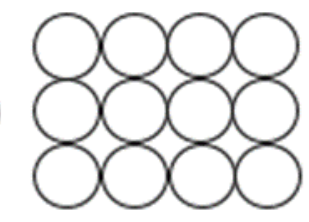

Porosity $=47.6 \%$ Bulk Density $=1.61 \mathrm{~g} / \mathrm{cm}^{3}$ (SG = 3.1) b)
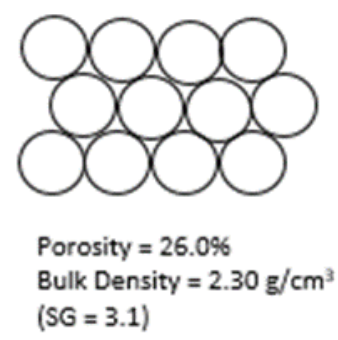

Figure 2.2: Particle Assemblies

are not uniform across long distances. Particles settle in different formations, which affects the density of particles and voids in a given space (Heiken et al., 1991). Figure 2.2 shows this relationship.

Relative density measures the degree of packing for the regolith sample relative to the regolith's minimum and maximum densities. It can also be expressed in terms of bulk density and porosity since both properties determine the amount of void space between particles given the relationship:

$$
\rho=S G * \rho_{w}(1-n)
$$

where $\rho$ is the measured bulk density, $S G$ is the specific gravity of a material, $\rho_{w}$ is the density of water, and $\mathrm{n}$ is the porosity. From this relationship the relative density, $\mathrm{D}_{\mathrm{R}}$, can be expressed as:

$$
\begin{gathered}
D_{R}=\frac{\rho_{\max }}{\rho} * \frac{\rho-\rho_{\min }}{\rho_{\max }-\rho_{\min }} \\
D_{R}=\frac{1-n_{\min }}{1-n} * \frac{n_{\max }-n}{n_{\max }-n_{\min }}
\end{gathered}
$$


Table 2.4: Best Averages for Lunar Regolith Bulk Density, Relative Density, and Porosity (After Houston et al. 1974, Heiken et al. 1991)

\begin{tabular}{|cccc|}
\hline Depth (cm) & $\begin{array}{c}\text { Bulk } \\
\text { Density } \\
\left(\mathbf{g} / \mathbf{c m}^{\mathbf{3}}\right)\end{array}$ & $\begin{array}{c}\text { Relative } \\
\text { Density (\%) }\end{array}$ & Porosity (\%) \\
\hline $0-15$ & $1.50 \pm 0.05$ & $65 \pm 3$ & $52 \pm 2$ \\
$0-30$ & $1.58 \pm 0.05$ & $74 \pm 3$ & $49 \pm 2$ \\
$30-60$ & $1.74 \pm 0.05$ & $92 \pm 3$ & $44 \pm 2$ \\
$0-60$ & $1.66 \pm 0.05$ & $83 \pm 3$ & $46 \pm 2$ \\
\hline
\end{tabular}

The physical properties of the lunar regolith were derived from lunar drill core samples from the Apollo missions. Table 2.4 shows the best averages derived for each parameter.

Data on the cohesion, compressibility, strengths and other mechanical properties was also generated from the regolith samples. Although mineral concentrations are important for construction and manufacturing purposes, extensive research has been done to confirm the existence of water on the moon. Two missions have independently shown that ice concentrations on the moon are known to exist in either permanently shadowed craters or within the surface regolith itself. The first mission, launched in 2008, involved the Indian Space Research Organization's (ISRO)'s satellite Chandrayaan-1, which was equipped with the Moon Mineralogy Mapper $\left(\mathrm{M}^{3}\right)$ spectrometer that measures the visible and near-infrared wavelengths that $\mathrm{OH}$ and $\mathrm{H}_{2} \mathrm{O}$ bonds readily absorb (Pieters et al., 2009). Results from $\mathrm{M}^{3}$, published in 2009, proved that water existed within 3 micrometers on the surface of the moon and indicated that the absorption features were strongest at higher latitudes; however, water concentration on the surface does not extend farther than a few millimeters (Pieters et al., 2009). In 2010, results from NASA's Lunar Crater Observation and Sensing Satellite (LCROSS) confirmed that ice exists in the permanently shadowed craters with water content of approximately $5.6 \pm 2.9 \%$ by weight (Colaprete et al., 2010).

\subsubsection{Summarized Information}

The information highlights topics relating to asteroid classification and the current state of Martian and Lunar surface characterization. The data collected from remote sensing and indirect testing as well as direct measurements on lunar regolith reveals valuable information about the mineralogical content and geologic history on the surfaces of these extraterrestrial bodies. These tests have not only identified mineral concentrations but also water concentrations. This information has directly led to developing ISRU technologies to extract and process these resources to provide for crews on long-term missions. Still, most of the data collected for martian and 
asteroid surfaces is from remote sensing techniques and meteorite analogs. Asteroid characterization is conducted by indirectly testing asteroids that are millions of miles away from Earth or by analyzing meteorites that have undergone significant chemical changes because they have passed through the Earth's atmosphere. The actual conditions and characteristics of individual asteroids may be significantly different when directly tested and the data collected so far can only reveal a limited amount of information. In similar fashion, Martian missions have determined mineralogy but cannot directly determine precise mechanical properties of samples. Table 2.5 lists the different means of obtaining surface data from Mars.

The Moon has the distinction of using direct testing methods on returned samples to determine content, and mechanical properties; however, samples were not characterized in-situ. Table 2.6 shows each sample return mission from the Moon with details of the mission with the number and mass of each sample returned.

Although spectrometers and remote sensing tools can determine the chemical composition of a sample, they cannot determine its mechanical properties. Terrestrial analogs can be used to estimate their mechanical properties, but the precise range is uncertain without direct testing methods. For asteroid missions, the frequencies of rubble-pile and monolithic asteroids are uncertain as well as the strength of these asteroids. The characterization testing will likely avoid destructive methods to prevent damage to target asteroids for spacecraft safety as well as to avoid damaging potentially valuable samples. 
Table 2.5: Spacecraft and Vehicles with Mineralogical Determination Capabilities (After Ehlmann and Edwards 2014)

\begin{tabular}{|c|c|c|c|}
\hline $\begin{array}{l}\text { Years of } \\
\text { Operation }\end{array}$ & $\begin{array}{l}\text { Instrument } \\
\text { Name }\end{array}$ & Location & Key Characteristics \\
\hline 1997-2006 & $\begin{array}{l}\text { Thermal } \\
\text { Emission } \\
\text { Spectrometer }\end{array}$ & Orbit & $\begin{array}{l}\text { Thermal infrared point } \\
\text { spectroscopy }(\sim 6-50 \mu \mathrm{m} ; 3 \\
\times 6 \mathrm{~km} \text { spots })\end{array}$ \\
\hline 2001-Present & $\begin{array}{l}\text { Thermal } \\
\text { Emission } \\
\text { Imaging } \\
\text { System }\end{array}$ & Orbit & $\begin{array}{l}\text { Thermal infrared } \\
\text { multispectral imaging } \\
\text { (6.7-14.8 um; } 7 \text { bands; } \\
\text { 100-m pixels) }\end{array}$ \\
\hline $\begin{array}{l}2004 \text { - 2011(Spirit) } \\
\text { 2004-Present } \\
\text { (Opportunity) }\end{array}$ & $\begin{array}{l}\text { Miniature } \\
\text { Thermal } \\
\text { Emission } \\
\text { Spectrometer }\end{array}$ & Rover & $\begin{array}{l}\text { Thermal infrared point } \\
\text { spectroscopy (landed; 5-29 } \\
\text { um; } 20 \text { mrad; i.e., } 20-\mathrm{cm} \\
\text { spots at } 10 \mathrm{~m} \text { ) }\end{array}$ \\
\hline 2004-Present & OMEGA & Orbit & $\begin{array}{l}\text { Visible/near-infrared } \\
\text { imaging spectroscopy } \\
(0.4-5.0 \mu \mathrm{m} ; 200-2,000 \\
\mathrm{m} / \text { pixel })\end{array}$ \\
\hline 2006-Present & $\begin{array}{l}\text { Compact } \\
\text { Reconnaissance } \\
\text { Imaging } \\
\text { Spectrometer } \\
\text { for Mars }\end{array}$ & Orbit & $\begin{array}{l}\text { Visible/near-infrared } \\
\text { imaging spectroscopy } \\
(0.4-4.0 \mu \mathrm{m} ; 18-200 \\
\mathrm{m} / \text { pixel })\end{array}$ \\
\hline $\begin{array}{l}2004 \text { - 2011(Spirit) } \\
\text { 2004-Present } \\
\text { (Opportunity) }\end{array}$ & $\begin{array}{l}\text { Mossbauer } \\
\text { Spectrometer }\end{array}$ & Rover & $\begin{array}{l}\text { Arm-based in situ } \\
\text { Mossbauer spectroscopy } \\
\text { (57Co source; 1.4-cm } \\
\text { footprint) }\end{array}$ \\
\hline $\begin{array}{l}\text { 2011-Present } \\
\text { (Curiosity) }\end{array}$ & $\begin{array}{l}\text { Chemistry and } \\
\text { Mineralogy }\end{array}$ & Rover & $\begin{array}{l}\text { Delivery of powdered } \\
\text { sample for X-ray diffraction } \\
(2 \vartheta=5-50)\end{array}$ \\
\hline
\end{tabular}


Table 2.6: Lunar Missions with Samples Returned

\begin{tabular}{|c|c|c|}
\hline Mission & Description & Sample Returned \\
\hline Apollo 11 & $\begin{array}{l}\text { July 20, } 1969 \text {; First manned } \\
\text { mission to the moon; } \\
\text { Landed in the Mare } \\
\text { Tranquillitatis }\end{array}$ & 58 samples weighing $21.6 \mathrm{~kg}$ \\
\hline Apollo 12 & $\begin{array}{l}\text { November 14, 1969; Landed } \\
\text { in Mare Cognitum }\end{array}$ & 69 samples weighing $34.3 \mathrm{~kg}$ \\
\hline Luna 16 & $\begin{array}{l}\text { September } 12,1970 ; \\
\text { Unmanned Soviet sample } \\
\text { return mission; Landed in } \\
\text { Mare Fecunditatis }\end{array}$ & 1 sample weighing 101 grams \\
\hline Apollo 14 & $\begin{array}{l}\text { January 31, 1971; Landed } \\
\text { in Fra Mauro }\end{array}$ & 227 samples weighing $42.3 \mathrm{~kg}$ \\
\hline Apollo 15 & $\begin{array}{l}\text { July 26, 1971; Landed in } \\
\text { Mare Imbrium }\end{array}$ & 370 samples weighing $77.3 \mathrm{~kg}$ \\
\hline Luna 20 & $\begin{array}{l}\text { February } 14,1972 ; \\
\text { Unmanned Soviet sample } \\
\text { return mission; Landed in } \\
\text { Mare Fecunditatis }\end{array}$ & 1 sample weighing 55 grams \\
\hline Apollo 16 & $\begin{array}{l}\text { April 16, 1972; Landed in } \\
\text { Descartes Highlands }\end{array}$ & 731 samples weighing $95.7 \mathrm{~kg}$ \\
\hline Apollo 17 & $\begin{array}{l}\text { December } 7,1972 \text {; Landed } \\
\text { in Taurus-Littrow valley }\end{array}$ & 741 samples weighing $110.5 \mathrm{~kg}$ \\
\hline Luna 24 & $\begin{array}{l}\text { August 9, 1976; Unmanned } \\
\text { Soviet sample return } \\
\text { mission; Landed in Mare } \\
\text { Crisium }\end{array}$ & 1 sample weighing 170 grams \\
\hline
\end{tabular}




\section{$2.2 \quad$ UPV Rock Characterization Technique}

\subsubsection{UPV Background}

The ultrasonic pulse velocity test is a non-destructive testing method that uses ultrasonic waves to characterize a material's elastic properties as well as to provide information about internal structure of a solid body. UPV has broad, well-researched

applications for terrestrial uses in civil, mechanical, and mining engineering that make it suitable for extraterrestrial rock characterization.

\subsubsection{Waveform Overview}

A mechanical or elastic wave is the propagation of a disturbance through a material body that is caused by the vibration of particles. The velocity the wave travels is dependent on the material type as well as the wave type and has the units length/time. The frequency of a wave is defined as the number of cycles of particle vibration per second. The frequency of a wave depends on the input source (e.g. impact, ultrasonics) and has the units Hertz $(\mathrm{Hz})$. The wavelength is defined as the distance between successive vibration peaks is shown by the symbol, $\lambda$. The velocity, frequency, and wavelength of a wave are related by:

$$
V=\lambda f=\frac{\Delta \mathrm{x}}{\Delta \mathrm{t}}
$$

The frequency of a wave also determines the amount of energy loss per unit of distance (attenuation) because as the number of vibrations per second increases, the more energy will be lost with each vibration. Lower frequencies also have longer wavelengths, which means the waves can travel farther, but they cannot detect smaller defects in a body. The attenuation of a wave is also dependent on the material. Homogeneous materials such as steel cause less wave attenuation than heterogeneous materials like concrete or rocks because these bodies are composed of different materials themselves as well as pore spaces which impede the path of a wave.

Two types of waves can propagate through a solid body: longitudinal waves or $\mathrm{P}$-waves, and transverse waves or S-waves. P-waves cause particle vibrations parallel to the direction that the wave is traveling, while S-waves cause vibrations perpendicular to the direction that the wave is traveling in. S-waves propagate perpendicular to the travel direction because they rely on the shear oscillation between particles. Consequently, S-waves travel slower than $\mathrm{P}$-waves and cannot travel through gases and liquids because fluids cannot shear.

The resistance to the propagation to a sound wave through the material is known as acoustic impedance. The acoustic impedance is a property of the material as 


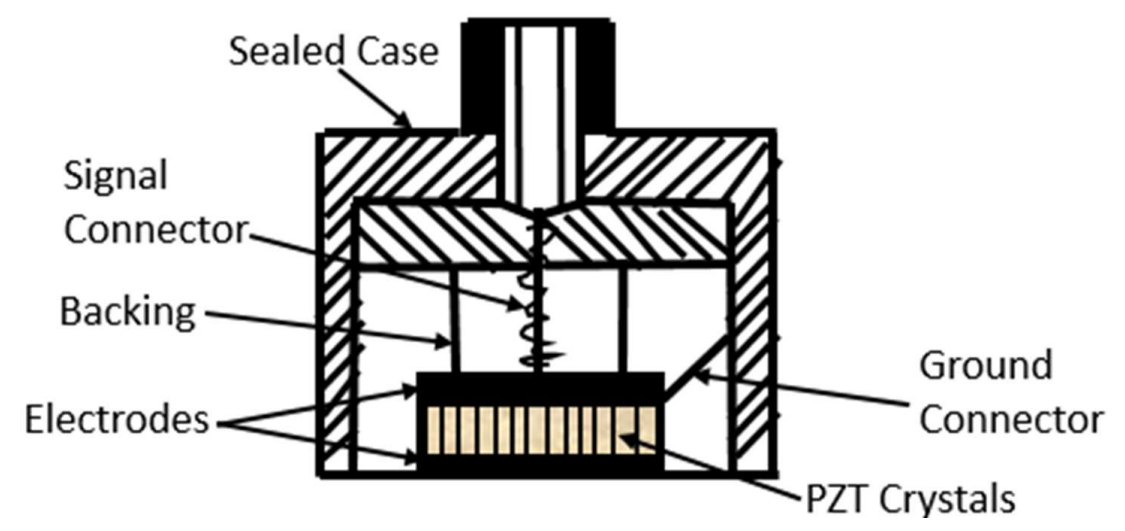

Figure 2.3: Transducer Cross-Section

defined by the material's density and the wave velocity through the material.

$$
Z=\rho V
$$

Acoustic impedance is especially important when a wave is propagating from one material to another because differences in impedances determine the amount of wave energy lost when the wave is at the boundary between the materials.

Refraction is another concern when passing an ultrasonic signal through a heterogeneous material. Refraction is the incomplete transmission of a wave through an interface. When a wave interacts with two materials that are not the same the wave is split in two and is said to be refracted. Part of this refracted wave is reflected and the other part is transmitted at a different angle. This angle of refraction is influenced by the angle of wave incidence.

\subsubsection{Data Acquisition}

Data acquisition units for UPV contain an oscilloscope, a electric pulser, and an amplifier. Connected to the DAQ are two transducers that act as the transmitter and the receiver. The transducers contain piezoelectric (PZT) crystals, which vibrate at a pre-determined frequency when stimulated by an electric signal and vice versa. The vibrations generate the sound wave that propagates through the medium. Figure 2.3 is an example of transducer cross section.

During a test, an electric pulse is first generated and sent to the transmitting transducer that in turn creates the ultrasonic wave that propagates through the medium. When the wave reaches the receiving transducer, the vibrations create an electric signal that is sent to through an amplifier. The purpose of the amplifier is to change the power and gain of the electric signal after it reaches the receiver. An amplifier can improve signal clarity but may amplify the noise in the waveform as well. Fi- 


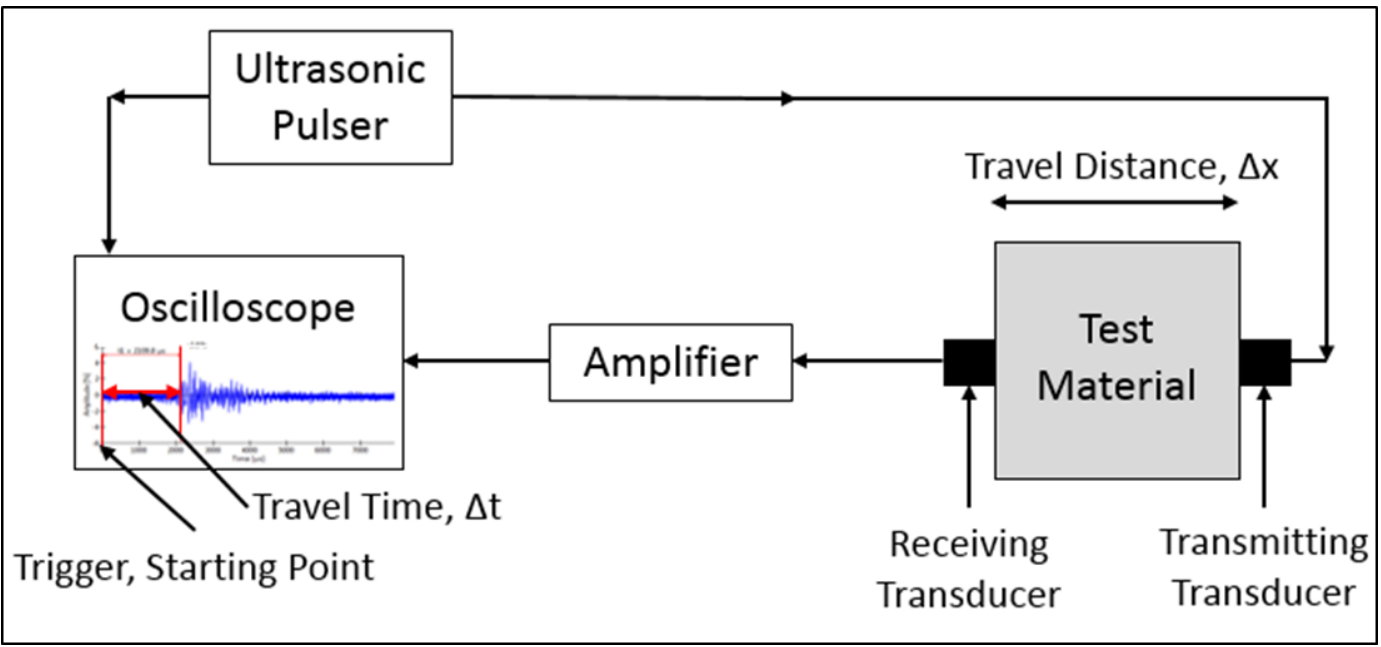

Figure 2.4: Example of UPV Structure

nally, the signal is received by an oscilloscope that displays the waveform. To improve the efficiency of the energy transfer from the transducer to the sample, a couplant is added to the ends of the transducers. The acoustic impedance of the couplant is crucial because the difference in acoustic impedances between materials determines the amount of energy transmitted. The ultimate goal of UPV this test obtain the time of arrival for a signal through a medium. Once the time of arrival is found, the length of the medium is divided by the time and thus yields the velocity of the wave through said medium. Figure 2.4 shows an example of a UPV setup.

\subsubsection{Data Utilization}

There are a multitude of ways to use wave velocity to characterize material. The following sections discuss fundamental and empirical methods to use wave velocities.

\section{Dynamic Moduli}

From the velocities of the $\mathrm{P}$ and $\mathrm{S}$ waves, $\mathrm{V}_{\mathrm{p}}$ and $\mathrm{V}_{\mathrm{s}}$ respectively, the dynamic moduli of the medium can be derived as long as the density of the medium is known a priori. The equations below list fundamentally proven relationships between ultrasonic velocities and the elastic properties of Young's modulus, the shear modulus, G, Poisson's ratio, $\nu$, and the bulk modulus, $\mathrm{K}$.

$$
\begin{gathered}
G=V_{s}^{2} \rho \\
\nu=\frac{V_{p}^{2}-2 V_{s}^{2}}{2\left(V_{p}^{2}-V_{s}^{2}\right)}
\end{gathered}
$$




$$
\begin{gathered}
E=\frac{\sigma}{\varepsilon} \\
E=V_{p}^{2} \rho \\
E=2 G(1+\nu) \\
K=-\frac{\Delta \mathrm{p}}{\nu} \\
K=\frac{E}{3(1-2 \nu)} \\
K=\rho\left(V_{p}^{2}-\frac{4}{3} V_{s}^{2}\right)
\end{gathered}
$$

where $\Delta \mathrm{p}$ is the change in compression on the sample, $\sigma$ is stress, and $\varepsilon$ is the strain. The properties indicate the elasticity, compressibility, and rigidness of a sample, which are important mechanical properties when discussing sample strength.

\section{Internal Structure}

The detection of discontinuities and pore spaces is possible due to the fact that ultrasonic waves cannot transmit across these voids. A propagating wave must find a path around the void, resulting in attenuation and an increase in the time arrival for the signal. If the velocity range of the medium is already well-established then slower velocities likely indicate the voids within the sample. Essentially, the slower the velocity is from a baseline value, the more voids within (McCann and Forde, 2001). Figure 2.5 is an example of the pathway of an ultrasonic wave when it encounters a void space.

Discontinuity detection is key for quality control of steel (ASTM Standard A388/ A388M-16a), concrete (ASTM Standard D6760-16) and rock (Aydin, 2015) because discontinuities significantly affect the strength of a specimen. Stress will propagate through these voids and cause fractures that will eventually lead to failure. Likewise, greater densities in solid material will generally yield faster wave velocities than less dense material since there are fewer pore spaces to avoid. 


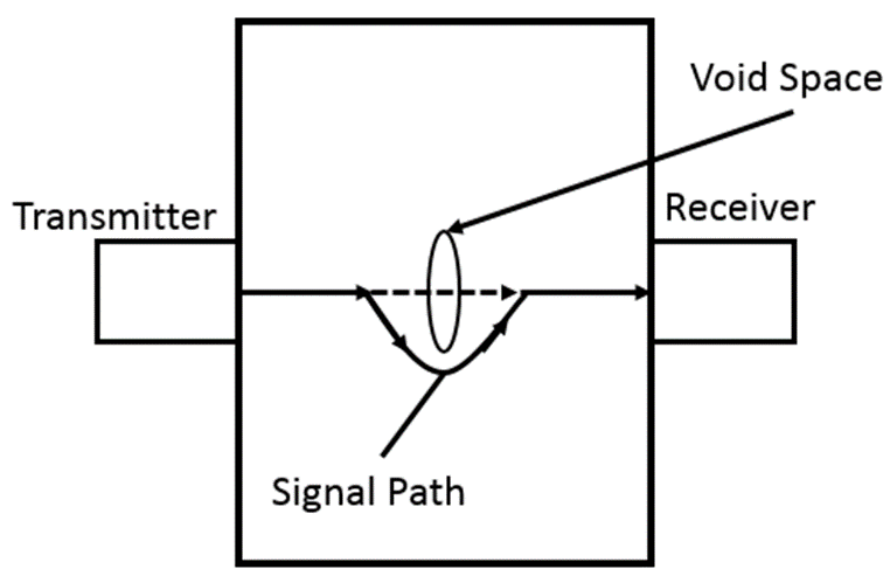

Figure 2.5: Void Space Example

\section{Strength Analogues}

Other uses for the longitudinal wave velocity include those that attempt to characterize the strength of a sample. Uniaxial compressive strength (UCS) is a common strength analogue within rock mechanics that designates the material's ability to withstand compressive stress. UCS is closely related to the stiffness and density of the material, which means the longitudinal velocity may provide a close approximation since it also depends on these properties. Numerous reports have attempted to define this relationship with Table 2.7 containing thirteen different equations. These equations are developed based on rock type, but many of them try to establish a broad relationship across several types to create a general relationship between UCS and $\mathrm{V}_{\mathrm{p}}$.

Eight of these equations use simple linear relationships to correlate P-wave velocity with compressive strength with remainder using power and exponential relationships. The coefficients of determination range from weak correlations at 0.53 to strong correlations at 0.96 ; however, the strongest relationships occur with relatively small sample sizes (Kiliç and Teymen, 2008; Khandelwal and Singh, 2009), which may provide strong correlations, but do not take into account as many nuances as larger sample sizes do. As a general rule, faster P-wave velocities correlate with larger compressive strength values. While numerous correlation equations are available, it is important to note that each equation relates P-wave velocity to UCS using site-specific data. Any equation that is empirically derived should be subjected to scrutiny regardless of the coefficient of determination. Differences not just in rock type, but in procedure for each study can alter analyses when using one of the equations. 
Table 2.7: Equations Relating P-wave Velocity to UCS

\begin{tabular}{|c|c|c|c|}
\hline Reference & Equation & $\begin{array}{l}\text { Coefficient of } \\
\text { Determination }\end{array}$ & $\begin{array}{c}\text { Sample } \\
\text { Description }\end{array}$ \\
\hline $\begin{array}{l}\text { Sharma and } \\
\text { Singh } 2008\end{array}$ & $U C S=0.0642 V_{p}-117.99$ & $\mathrm{R}^{2}=0.90$ & $\begin{array}{l}49 \text { samples of } \\
\text { different rock } \\
\text { types }\end{array}$ \\
\hline $\begin{array}{l}\text { Kahraman } \\
2001\end{array}$ & $U C S=9.95 V_{p}^{1.21}$ & $\mathrm{R}=0.83$ & $\begin{array}{c}27 \text { samples of } \\
\text { limestone, } \\
\text { sandstone, and } \\
\text { marls }\end{array}$ \\
\hline $\begin{array}{l}\text { Moradian and } \\
\text { Behnia } 2009\end{array}$ & $U C S=165.05 e^{\left(\frac{-4.452}{V_{p}}\right)}$ & $\mathrm{R}^{2}=0.7$ & $\begin{array}{c}64 \text { samples of } \\
\text { limestone, } \\
\text { sandstone, and } \\
\text { marl }\end{array}$ \\
\hline $\begin{array}{l}\text { Khandelwal } \\
2013\end{array}$ & $U C S=0.033 V_{p}-34.83$ & $\mathrm{R}^{2}=0.87$ & $\begin{array}{l}12 \text { samples of a } \\
\text { wide rock types }\end{array}$ \\
\hline $\begin{array}{l}\text { Khandelwal } \\
\text { and Singh } \\
2009\end{array}$ & $U C S=0.1333 V_{p}-227.19$ & $\mathrm{R}^{2}=0.96$ & $\begin{array}{c}12 \text { different rock } \\
\text { samples }\end{array}$ \\
\hline $\begin{array}{l}\text { Minaeian and } \\
\text { Ahangari } 2013\end{array}$ & $U C S=0.005 V_{p}$ & $\mathrm{R}^{2}=0.94$ & $\begin{array}{c}\text { Some samples of } \\
\text { weak } \\
\text { conglomeratic } \\
\text { rock }\end{array}$ \\
\hline $\begin{array}{l}\text { Diamantis } \\
\text { et al. } 2009\end{array}$ & $U C S=0.11 V_{p}-515.56$ & $\mathrm{R}^{2}=0.81$ & $\begin{array}{l}32 \text { samples of } \\
\text { serpentinite rock }\end{array}$ \\
\hline $\begin{array}{l}\text { Çobanoglu and } \\
\text { Çelik } 2008\end{array}$ & $U C S=56.71 V_{p}-192.93$ & $\mathrm{R}^{2}=0.67$ & $\begin{array}{l}150 \text { samples of } \\
\text { sandstone, } \\
\text { limestone, and } \\
\text { cement mortar } \\
\text { rock cores }\end{array}$ \\
\hline $\begin{array}{l}\text { Entwisle et al. } \\
2005\end{array}$ & $U C S=0.78 e^{0.88 V_{p}}$ & $\mathrm{R}^{2}=0.53$ & $\begin{array}{l}142 \text { samples of } \\
\text { Volcanic rock }\end{array}$ \\
\hline $\begin{array}{l}\text { Mishra and } \\
\text { Basu } 2013\end{array}$ & $U C S=0.087 V_{p}-355.8$ & $\mathrm{R}^{2}=0.72$ & $\begin{array}{l}20 \text { samples of } \\
\text { granite }\end{array}$ \\
\hline $\begin{array}{l}\text { Mishra and } \\
\text { Basu } 2013\end{array}$ & $U C S=0.05 V_{p}-126.4$ & $\mathrm{R}^{2}=0.82$ & $\begin{array}{l}20 \text { samples of } \\
\text { sandstone }\end{array}$ \\
\hline $\begin{array}{l}\text { Tugrul and } \\
\text { Zarif } 1999\end{array}$ & $U C S=35.54 V_{p}-55$ & $\mathrm{R}=0.80$ & $\begin{array}{l}19 \text { samples of } \\
\text { granitic rocks }\end{array}$ \\
\hline $\begin{array}{l}\text { Kiliç and } \\
\text { Teymen } 2008\end{array}$ & $U C S=2.304 V_{p}^{2.4315}$ & $\mathrm{R}^{2}=0.94$ & $\begin{array}{c}19 \text { samples of } \\
\text { different rock } \\
\text { types }\end{array}$ \\
\hline
\end{tabular}


Table 2.8: Normal Laboratory Testing Conditions (After Aydin, 2015; ASTM Standard D2845-08)

\begin{tabular}{|c|c|c|}
\hline Parameter & Standard & Relevant in Space \\
\hline Sample geometry & Smooth, flat, and parallel faces & No \\
\hline Transducer frequency & $50-500 \mathrm{kHz}$ & Yes \\
\hline Coupling medium & $\begin{array}{l}\text { Thin layer between transducers } \\
\text { and sample }\end{array}$ & No \\
\hline Coupling Sstress & $\begin{array}{l}\text { Small stress }(\sim 10 \mathrm{kPa}) \text { for a } \\
\text { given transducer diameter }\end{array}$ & Yes \\
\hline Transudcer alignment & Normal to both faces & Yes \\
\hline Moisture content & $\begin{array}{l}\text { Either dry, fully satured, or } \\
\text { in-situ }\end{array}$ & No \\
\hline Wave travel distance & $10 \mathrm{x}$ the average grain size & No \\
\hline
\end{tabular}

\subsubsection{Environmental and In-Situ Testing Issues}

For UPV testing in general, predicting in-situ properties based on laboratory test results is complicated due to inadequate representation of field conditions (Aydin, 2015). Laboratory conditions will not be present in-situ and much less so on an extraterrestrial body. From ASTM and ISRM standards (ASTM Standard D2845-08 and Aydin, 2015), Table 2.8 lists normal testing conditions for UPV laboratory tests and the relevancy for space testing.

At least four of these of these testing conditions will not reflect in-situ space conditions: (1) Sample dimension restriction (2) sample surface roughness control (3) coupling medium selection (4) moisture content. These standards also do not take into account any regolith lying on the surface of the sample. Sample preparation tools will likely not be part of an extraterrestrial a rock characterization equipment set due to space restrictions on board a spacecraft. Standard protocols will have to be modified for conditions found in space. Previous reports have found protocol adjustments to into account robotic alignment issues and sub-zero temperatures, but have not taken into account some physical properties of the samples (Goodman, 2016; Savage, 2016).

\section{Sample Dimensions}

In laboratory tests, sample dimensions are typically small (10 - $15 \mathrm{~cm}$ in length) to avoid signal attenuation problems through larger samples. Alternatively, from ARM mission parameters, the target sample size for a boulder is 2 - 4 meters (Mazanek et al., 2015), which is much larger than normal sample sizes used during rock testing. Signal attenuation is a well-known phenomenon, but must be assessed with these 
mission requirements. Depending on the frequency of the transducer, UPV can be conducted on any specimen without limitations on shape or dimensions as long as the test can provide a distinguishable waveform, with higher frequencies favoring shorter sample lengths (Aydin, 2015). For UPV to be considered part of the ARM characterization testing suite, the effects of sample length must be investigated.

\section{Surface Roughness}

Standard UPV testing protocol from International Society of Rock Mechanics (ISRM) states that sample preparation requires test specimens with smooth, flat, and parallel faces because an uneven surface cannot uniformly distribute waves across the surface. (Aydin, 2015). The roughness of the sample surface is an in-situ physical property that is removed during laboratory preparation; however, in-situ testing will not prepare samples to the same degree as for laboratory testing, if any at all. As a result, the signal attenuation attributed to limited contact area must be investigated. Since standard testing protocol mandate smooth surfaces, very few studies have reported on the influence of surface roughness on signal attenuation. Nevertheless, Kahraman (2002) found that increasing fracture roughness within samples decreases $\mathrm{P}$-wave velocity. His report used five samples from three different rock types with different fracture roughness coefficients 0 through 4 with 0 meaning no roughness to 4 meaning very high roughness. Across all three rock types, the P-wave velocity decreased with increasing roughness.

Even though signal attenuation attributed to surface roughness is not well-researched in rock mechanics, it is discussed within research regrading quality control for metal products. While rocks normally have rougher profiles than machined metal, the concept of surface roughness is similar between them. Nagy and Adler (1987); Nagy and Rose (1993); and Oh et al. (1994) all state that increasing surface roughness increases signal attenuation in metal testing. Nagy and Adler (1987) found that immersing the transducers and sample within a water bath can mitigate most of the signal attenuation attributed to surface roughness while in Nagy and Rose (1993) found that the effects of surface roughness becomes negligible below a certain frequency for a certain degree of roughness. Oh et al. (1994) used ultrasonic techniques to measure surface roughness and compared the results to those gathered with a profilometer, finding that ultrasonic waves can accurately assess the degree of surface roughness on a sample.

\section{Lack of Gel Couplant}

A layer of couplant applied during testing ensures efficient energy transfer between the transducers and the sample. A large variety of couplant options includes grease, 
glycerin, putty, Vaseline, and oil (Aydin, 2015). Although a gel couplant is normally used during testing to improve energy transmission, it may not be included as part of an ultrasonic equipment set for in-situ rock characterization because its utility may be limited by property changes due to extreme temperatures encountered in space. While prior tests without couplant are sparse, Blomme et al. (2002); Gan et al. (2001); Grandia and Fortunko (1995) show that air can act as an effective coupling medium with alterations to the UPV equipment for quality control of metal products; however, the atmosphere on the Moon, Mars, or asteroids are not pressurized to the same degree on Earth. The lack of an atmosphere means that an air couplant will not be an effective coupling medium methods especially since the methods discussed do not involve sample contact. Essentially, ultrasonic waves will be unable to reach the sample while attempting to use air-couplant in space.

\section{Regolith Testing}

Another environmental concern that must be addressed is the regolith that covers the surface of extraterrestrial bodies. If the tools to remove it are not provided, then its effect on signal attenuation needs to be taken into account. Previous ultrasonic testing on terrestrial soils has yielded correlations between correlations between dynamic moduli and compaction such as in Inci et al. (2003); Weidinger et al. (2009); Yesiller et al. (2000). The effects of soil saturation on dynamic moduli have also been investigated (Yesiller et al., 2000; Lawrence Jr, 1965; Stephenson, 1978; Weidinger et al., 2009). Their findings show that the amount of voids as well as the water content will significantly affect the wave velocity and subsequently the dynamic moduli. Generally, as compaction increased, wave velocity increased and as saturation increased, the wave velocity decreased. The reports do not mention correlations between the size of the particles used during testing. ISRM standards mention the grain size of samples having an effect on wave velocity (Aydin, 2015). In order to assess the impact of the surface regolith, all physical parameters that dictate a regolith mixture must be addressed.

\section{Trapped Frozen Water}

Ice may be trapped within the surface regolith of extraterrestrial bodies. Terrestrially, the effect of ice on ultrasonic waves has been measured in order to determine the dynamic moduli and mechanical properties of frozen rock and soil samples. Several studies have examined the change in properties as a function of temperature (Timur, 1968; Wang et al., 2006; Park et al., 2013; Christ and Park, 2009) or a function of porosity and degree of melting (Zimmerman and King, 1986). Nakano et al. (1972) have measured P- and S-waves in water saturated frozen soils and suggested that 
the decrease in $\mathrm{P}$-wave velocities was due to the physical change from water to ice. Nakano and Arnold (1973) investigated the effect of ice saturation in frozen sand on the ultrasonic wave velocities found that the wave velocities increased with ice saturation. Wang et al. (2006); Park et al. (2013) found differences in soils and freezing temperatures have direct effects on dynamic elastic constants. Although, the research is extensive, the effects of particle size and distributions on the wave velocities within these mixtures has not been investigated. Most samples were representative of the area they were collected from so the particle size distributions were not varied during testing.

\subsubsection{Summary}

UPV is a standard non-destructive test commonly used to determine dynamic moduli of materials. The velocity of ultrasonic waves is dependent on the mechanical and physical properties of the sample being tested. The wave velocities can also be used to qualitatively determine the internal structure of a sample as well as provide empirical estimations for mechanical properties such as compressive strength. These tests are normally conducted in laboratory settings; however, the conditions that will be encountered on extraterrestrial bodies will require adjustments to standard procedures to take into account changes in physical sample properties. From ISRM standards, UPV tests can be conducted on any specimen without limitations on shape or dimensions as long as the test can provide a distinguishable waveform. The roughness of the sample surface is a physical property that is removed during preparation. Research regarding joint fractures and metal machining suggests that increasing roughness will increase signal attenuation. The utility of a gel couplant is questionable due to the extreme temperatures that will be encountered. Literature shows that the surrounding atmosphere can act as an effective coupling medium. Research regarding regolith suggests that as regolith compaction increased, wave velocity increased and as saturation increased, the wave velocity decreased. The literature does not mention the effects of particle size while UPV standards note the effect of grain sizes in a rock sample. Finally, frozen water trapped within surface regolith will be a valuable resource for ISRU efforts. Previous work has found that wave velocities increase with ice saturation and differences in soils and freezing temperatures have direct effects on dynamic elastic constants. The takeaways from this section of the literature review are:

- Testing must assess the influence of sample dimensions, roughness and regolith cover as well as the absence of a gel couplant.

- For UPV to be considered part of the ARM characterization testing suite, a 
mitigation strategy for sample length must be devised.

- In order to assess the impact of the surface regolith, all physical parameters that dictate a regolith mixture must be addressed.

- Mitigation strategies for surface roughness exist for quality control in metal machining meaning a strategy can developed for rock mechanics.

- Further research is required to assess UPV's ability to characterize ice concentrations with various distributions of regolith particle sizes.

Table 2.9 lists five conditions that are excluded during a laboratory test along with citations of previous work and a brief description of each condition.

Table 2.9: UPV In-Situ Testing Conditions

\begin{tabular}{|c|c|c|}
\hline Condition & Description & Citations \\
\hline Sample Dimensions & $\begin{array}{l}\text { The length of the sample } \\
\text { will determine the amount } \\
\text { of signal attenuation that } \\
\text { occurs within a sample. }\end{array}$ & $\begin{array}{l}\text { (Aydin, 2015; ASTM } \\
\text { Standard D2845-08) }\end{array}$ \\
\hline Surface Roughness & $\begin{array}{l}\text { The natural surface texture } \\
\text { of the sample limits the } \\
\text { amount of contact area } \\
\text { between the sample and } \\
\text { the transducers. }\end{array}$ & $\begin{array}{l}\text { (Kahraman, 2002; } \\
\text { Nagy and Adler, 1987; } \\
\text { Nagy and Rose, 1993; } \\
\text { Oh et al., 1994) }\end{array}$ \\
\hline Surface Regolith & $\begin{array}{l}\text { The particles lying on the } \\
\text { surface of the sample can } \\
\text { cause additional signal } \\
\text { attenuation before the } \\
\text { waves reach the sample } \\
\text { beneath. }\end{array}$ & $\begin{array}{l}\text { (Yesiller et al., 2000; } \\
\text { Weidinger et al., 2009; } \\
\text { Stephenson, 1978; } \\
\text { Slavova et al., 2010; } \\
\text { Lawrence Jr, 1965; } \\
\text { Inci et al., 2003) }\end{array}$ \\
\hline Lack of Coupling Gel & $\begin{array}{l}\text { Possibility of no gel } \\
\text { medium on board the } \\
\text { spacecraft due to physical } \\
\text { changes consequential of } \\
\text { sub-zero temperatures. }\end{array}$ & $\begin{array}{c}\text { (Blomme et al., 2002; } \\
\text { Gan et al., 2001; } \\
\text { Grandia and } \\
\text { Fortunko, 1995) }\end{array}$ \\
\hline $\begin{array}{l}\text { Frozen Water } \\
\text { Trapped within } \\
\text { Regolith }\end{array}$ & $\begin{array}{c}\text { Water may be trapped } \\
\text { within regolith particles on } \\
\text { the surface. }\end{array}$ & $\begin{array}{l}\text { (Timur, 1968; Wang } \\
\text { et al., 2006; } \\
\text { Zimmerman and King, } \\
\text { 1986; Park et al., } \\
\text { 2013; Nakano et al., } \\
\text { 1972; Christ and Park, } \\
\text { 2009) }\end{array}$ \\
\hline
\end{tabular}




\section{Chapter 3}

\section{Materials and Methods}

\subsection{Experimental Objectives}

An experimental program was implemented to address the influence of sample size, surface regolith, surface roughness, and couplant on the transmission of P-waves. Thus, three test series were developed for each physical property to establish relationships between changes in wave velocity and each property in order to develop a procedure to mitigate their influence. The length and roughness testing took into account changes in velocity depending on the addition of gel couplant, while the regolith test series attempted to provide an in-situ replacement for gel couplant. An additional test series was developed to investigate the ability of UPV to characterize changes in ice concentrations with variable particle size distributions was also evaluated. In total, four separate test series were developed.

\subsection{Sample Procurement}

\subsubsection{Rock Samples}

Available limestone and chalk were used to form the main body of regolith and roughness testing. The limestone samples were extracted from the Elbrook and Conococheague formations in northern Virginia and the chalk samples were cored from the Austin formation in Texas. Limestone is a sedimentary rock mostly made of calcium carbonate crystals $\left(\mathrm{CaCO}_{3}\right)$. It also contains various amounts of $\mathrm{Mg}$, Fe, or $\mathrm{Zn}$ atoms arranged in alternating layers with carbonate. Limestone forms mostly from dead calcacerous organisms that sink to the bottom of a body water and create layers of calcium carbonate. Over time the detritus is compressed and forms into limestone or other forms such as chalk. Chalk is contains a higher concentration of calcium carbonate than limestone, but varying amounts of quartz and clay. Chalk also has a 
Table 3.1: Baseline Data for Chalk and Limestone (After Savage, 2016)

\begin{tabular}{|lccc|}
\hline Property & Unit & Chalk & Limestone \\
\hline Density & $\left(\mathrm{g} / \mathrm{cm}^{3}\right)$ & $1.86-2.02$ & $2.60-2.77$ \\
P-Wave Velocity & $(\mathrm{m} / \mathrm{s})$ & $2,713-4,099$ & $5,071-7,049$ \\
UCS & $(\mathrm{MPa})$ & $10.6-15.5$ & $102.6-115.2$ \\
\hline
\end{tabular}

lower density and a greater porosity than limestone. Sample length varied between 7.6 and $15 \mathrm{~cm}$ while every the sample diameter was around $4.8 \mathrm{~cm}$. Figure 3.1 shows limestone and chalk rock cores together. The samples were characterized in Savage (2016) and the baseline data for chalk and limestone is displayed in Table 3.1.

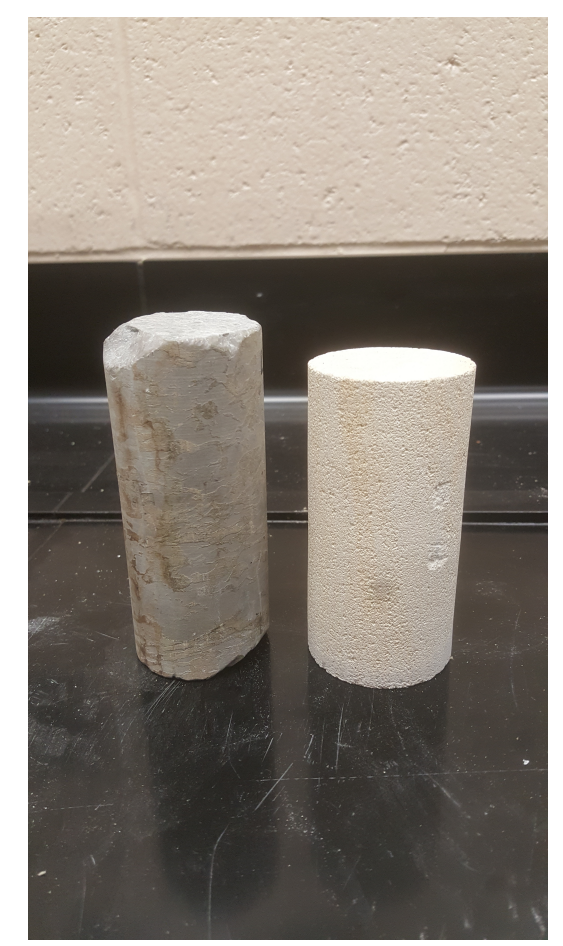

Figure 3.1: Limestone (L) and Chalk (R) Cores

\subsubsection{Concrete Samples}

In addition to the rock samples, three concrete beams at lengths of $1 \mathrm{~m}, 3 \mathrm{~m}$, and $6 \mathrm{~m}$ were constructed for the length testing series. The dimensions were selected because the mission parameters of the ARRM had initially stipulated a target boulder length of 2 - $6 \mathrm{~m}$. The concrete mix was purchased from Central Supply Co. located in Morgantown, WV and the mix was poured into wooden molds lined with a lubricant to prevent adherence to the mold. The concrete mix was rated at approximately 27 - $28 \mathrm{MPa}$. Immediately after the pouring, the sides of the molds were tapped with 


Table 3.2: Concrete Baseline Values
\begin{tabular}{|lcc|}
\hline Property & Unit & Value \\
\hline P-Wave & $\mathrm{m} / \mathrm{s}$ & 4,845 \\
S-Wave & $\mathrm{m} / \mathrm{s}$ & 2,761 \\
Density & $\mathrm{g} / \mathrm{cm}^{3}$ & 2.28 \\
Poisson's & ---- & 0.259 \\
Young's Modulus & $\mathrm{GPa}$ & 43.85 \\
Strength & $\mathrm{MPa}$ & 27.35 \\
\hline
\end{tabular}

rubber mallets to remove air pockets trapped within the concrete mix. After one day of open air cure, burlap cloth was placed over the beams to control the moisture content. The total cure time for all beams was 28 days. Each day, at approximately the same time, water was applied to the beam top surfaces to soak into the burlap cloth and maintain moisture cure levels. Figure 3.2 shows the beams immediately after the pour. Additionally, concrete was poured into 9 cylinder molds with lengths of $30.5 \mathrm{~cm}$ and diameters of $15.25 \mathrm{~cm}$. The cylinders were tested to supply baseline values for the concrete mixture. The cores were cured for different times and their mechanical properties were evaluated over time. Table 3.2 shows the baseline data after 28 days of cure time.

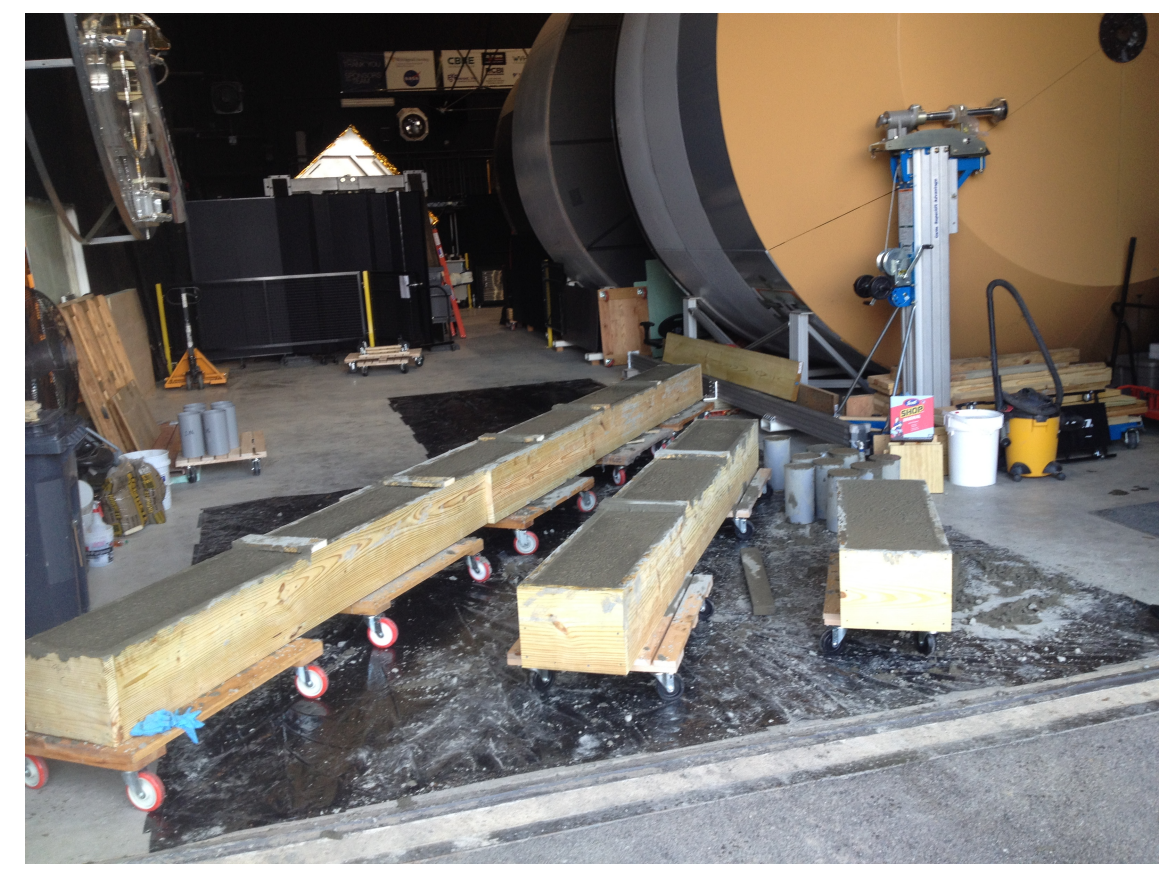

Figure 3.2: Concrete Beams Immediately After Pour. From Left to Right: 6 m, 3 m, and $1 \mathrm{~m}$ beams 


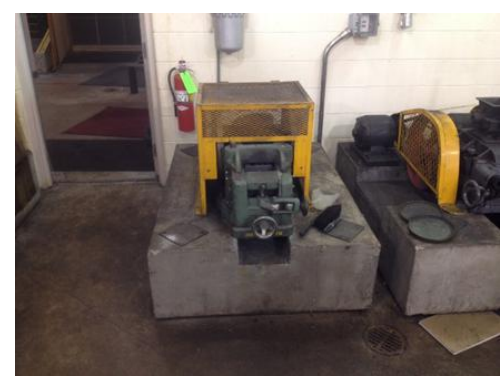

Sturtevant Jaw Crusher

1) Two passes through crusher

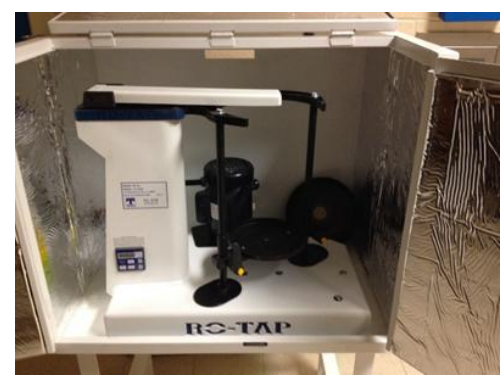

Ro-Tap RX-29

3) 15 - 20 minutes of dry sieving

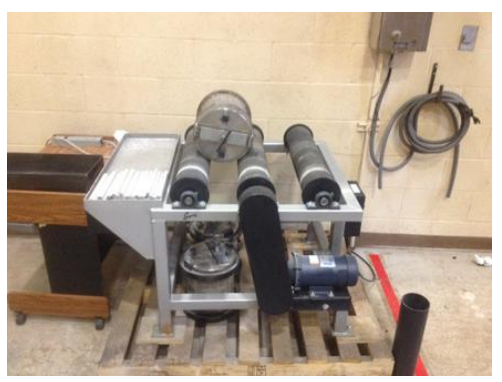

Sepor Ball Mill

2) 15 minutes of grinding

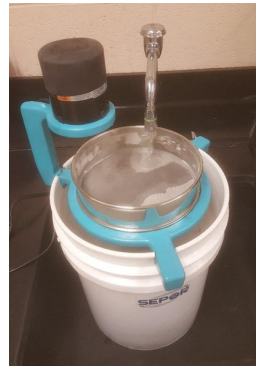

Sepor Sieve Shaker

4) 20 - 30 minutes of wet sieving

Figure 3.3: Regolith Generation Equipment

\subsubsection{Regolith Generation}

To generate regolith simulant, limestone and chalk cores were staged crushed and ground in an Sturtevant jaw crusher and a Sepor ball mill. After grinding, material was dry sieved using No. 10, 18, 35, 60 and 100 sieves. Wet screening was used for sizes less than 150 microns. The equipment used for this procedure are shown in Figure 3.3.

The particle size classes were chosen based on the NASA Lunar Sourcebook (Heiken et al., 1991). Figure 3.4 shows the core and regolith size classes.

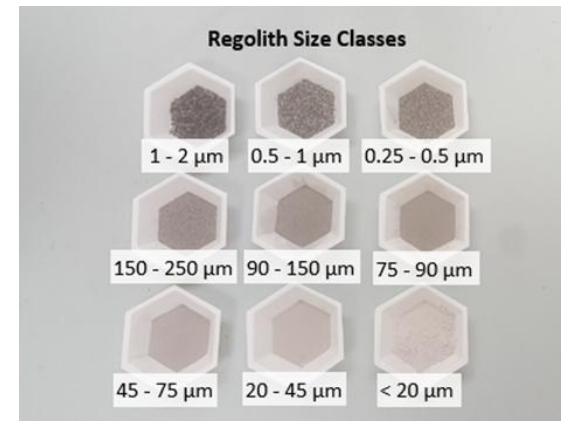

Figure 3.4: Regolith Size Classes 


\subsection{Methods/Procedures}

\subsubsection{Standard Test Protocols for UPV}

The standard test procedures were based on ISRM standards (Aydin, 2015). For

all test series, the ultrasonic platform that was used was the Proceq(C)Pundit-Lab 200 with $54 \mathrm{kHz}$ transducers used for all tests.

\subsubsection{Data Processing}

UPV operates by turning an electrical signal into an ultrasonic wave that is sent back to receive a waveform on an oscilloscope. The length of the sample is divided by the time it takes for the signal to arrive at the receiving transducer to calculate the velocity for a specific medium. In addition to the arrival time, wave amplitude was measured as a surrogate for signal quality.

\subsubsection{Length Testing}

To assess the influence of sample length, three large concrete beams were constructed, UPV was measured as a function of length down the beam. A combination of direct and semi-direct testing methods was used to gather data at numerous lengths. This data was compared to the baseline velocity value from the concrete cores. Figure 3.5 shows the different transmission methods.

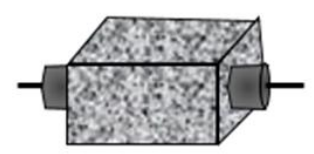

Direct

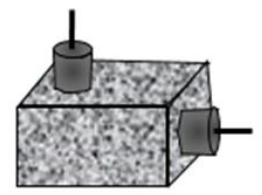

Semi-Direct

Figure 3.5: Methods of Transmission

\subsubsection{Regolith Testing}

To assess the influence of a regolith cover, the effects of different regolith parameters on wave velocity and amplitude were evaluated. These four parameters to be used for testing were: particle top size, particle size distribution, compaction, and regolith depth. The experiments used crushed limestone sorted into nine different size classes from 20 to 2000 microns in particle diameter and a single limestone core was used to act as the sample. Attached to the core was a piece of PVC to hold the regolith and the baseline velocity of the core was measured at $5300 \mathrm{~m} / \mathrm{s}$. Figure 3.6 shows the core sample. 


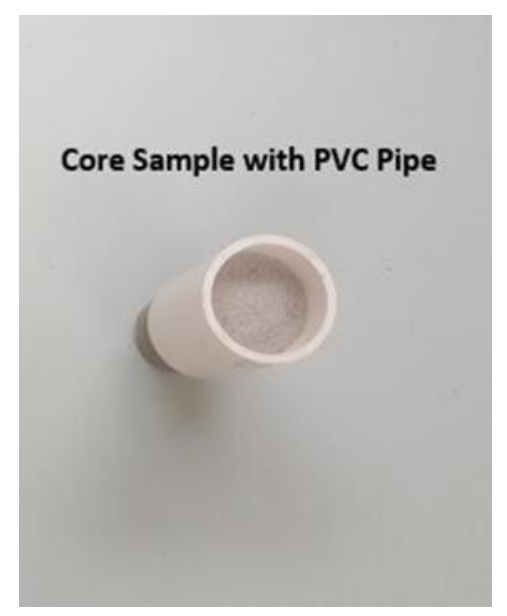

Figure 3.6: Limestone Core

The size distribution and top size for each unique blend were used in a GaudinSchumann equation to derive the mass of regolith in each size class. The blend was poured onto the limestone core and the amount depended on the depth and compaction required for that test. The depth was then checked before and after compaction to ensure this value remained constant. The transmitter was then be placed lightly on top of the regolith and the wave velocity was measured at least 10 times per test. All tests for each blend were completed in the same fashion. The mix was then wet sieved and deconstructed to have enough material for the next blend.

In the experimental design, the particle size distribution was defined using a Gaudin-Schumann equation. The equation is frequently used in mineral processing to represent the distribution of particles sizes in a blend Wills and Napier-Munn (2015). The Gaudin-Schumann equation is:

$$
Y=\left(\frac{x}{b}\right)^{k}
$$

where $\mathrm{x}$ is particle size, $\mathrm{y}$ is the cumulative percent mass passing, $\mathrm{b}$ is the particle top size, and $\mathrm{k}$ is the size distribution modulus. $\mathrm{B}$ and $\mathrm{k}$ are used in the experimental design to represent the different blends used for testing. $b$ is the maximum particle size in the blend. As $\mathrm{k}$ increases from zero, the homogeneity increases to the point where all particles are in the top size class. Compaction describes how densely packed particles are, however, not all particle size distributions allow for the same percent of compaction. For a certain particle size distribution, compaction values range between no compaction and a specific point of incompressibility. For this test series, the values used for compaction were classified as: "none", "some", and "maximum". A compaction ratio denoted as "some" is a qualitative assessment based on the maximum compaction required for a specific blend. As long as the compaction was less than the maximum, 
it was considered acceptable. Percent compaction is given by the equation below:

$$
\% \text { Compaction }=\frac{\left(z_{0}-z_{1}\right)}{z_{0}} * 100
$$

where $z_{0}$ is the original height of the regolith and $z_{1}$ is the compacted height. $Z_{1}$ is also the final parameter: regolith depth.

The first regolith series (R1) attempted to establish statistical significance to the four parameters that make up the physical properties of surface regolith. Table 3.3 shows the different values for each parameter.

Table 3.3: Parameters for Series 1 of Regolith Testing

\begin{tabular}{|l|ccc|}
\hline \multirow{2}{*}{ Parameter } & \multicolumn{3}{|c|}{ Parameter Values } \\
& Low & Medium & High \\
\hline Top Size $(\mu \mathrm{m})$ & 500 & 1000 & 2000 \\
Distribution Modulus & 0.1 & 0.5 & 1.0 \\
Compaction Ratio & None & Some & Maximum \\
Maximum Depth $(\mathrm{cm})$ & 1 & 2 & 3 \\
\hline
\end{tabular}

The second series of regolith testing (R2), attempted to simplify the procedure from R1 and and provide results that would help establish a relationship between regolith depth and wave velocity as well as isolate the influence of particle size on signal attenuation. For this series, the procedure only used three parameters but effectively retain the same procedure. Particle top size and distribution modulus were unchanged; however, compaction was kept at maximum for all tests and depth was changed to mass. This eliminated compaction as a variable and allowed a simpler method of determining the height of the regolith. Maximum compaction was achieved by placing a $1 \mathrm{~kg}$ calibration weight on top of the mass of regolith and $14.2 \mathrm{~kg}$ weight was placed on top of the calibration weight. Across the $4.8 \mathrm{~cm}$ diameter of the limestone core the pressure on the regolith was $\sim 83 \mathrm{kPa}$ or $12 \mathrm{psi}$. The mass of each test was either 15,30 , or 45 grams, which yielded regolith depths between $0.25 \mathrm{~cm}$ and $1.2 \mathrm{~cm}$. A full factorial experimental design was used instead of a Box-Benekhen to provide the maximum possible amount of data points. The parameters for R2 can be seen in Table 3.4.

Table 3.4: Parameters for Series 2 of Regolith Testing

\begin{tabular}{|l|ccc|}
\hline \multirow{2}{*}{ Parameter } & \multicolumn{3}{|c|}{ Parameter Values } \\
& Low & Medium & High \\
\hline Top Size (jm) & 500 & 1000 & 2000 \\
Distribution Modulus & 0.1 & 0.5 & 1.0 \\
Regolith Mass (g) & 15 & 30 & 45 \\
\hline
\end{tabular}


The purpose of the last three series was to further isolate the change in wave velocity as particle top size and regolith depth changed. For the third regolith series (R3), only top size and regolith mass were considered. As such, only individual size classes were used for testing. All 9 size classes were used, and only 3 points were collected for each class using the same regolith masses in R2. The fourth series (R4) used only three size fractions. 45, 500, and $2000 \mu \mathrm{m}$, but regolith was continuously added in 5 gram increments until distinct signals could not be recognized. The fifth series (R5), used one size class of chalk particles (1000-2000 $\mu \mathrm{m}$ ) and was placed on top of the same limestone core in previous testing. The regolith was added in $5 \mathrm{~g}$ increments until distinct signals could not be recognized. This data was then compared to data collected from R4 with the same particle size class.

Prior to the first regolith testing series, an experimental design was constructed to efficiently test different blends. Each regolith parameter was assigned a high, medium, and low value, thus a full factorial test required 81 different tests to be conducted. A fractional factorial or a central composite rotatable design required fewer experiments to arrive at the same conclusions. Therefore, fractional factorial and central composite rotatable designs are considered to be more time-efficient than full-factorial designs (Amini et al., 2016). Specifically a Box-Behnken design was used for the regolith testing series. The design yielded a test matrix with 27 points; greatly minimizing the amount of time spent collecting data. The second regolith series utilized a full factorial experimental design because the test matrix required only 27 points so the same amount of time was spent testing as the previous series.

\subsubsection{Roughness Testing}

The purpose of surface roughness testing was to evaluate signal attenuation as a wave encounters an uneven surface when it is being transmitted to the testing media. The uneven surface limits the amount of area the transducer is in contact with, and means that a certain portion of ultrasonic waves have to pass through air to reach the medium. This transmission causes an energy loss that has to be taken into account when attempting to quantify signal attenuation without the use of a gel couplant. Work from previous studies has shown the influence of alignment between transducers (Goodman, 2016); thus, the transducers have to be parallel to each other in order to isolate signal attenuation through a rough surface.

Finding a natural sample of limestone with two parallel rough ends was prohibitively difficult, so procedure to artificially generate rough, parallel surfaces was implemented. To prepare for testing, eight limestone samples were created from three parent cores. Quarter-inch grooves were cut around the bodies of the three parent cores because stress propagates through the weakest points of a body. A load was 


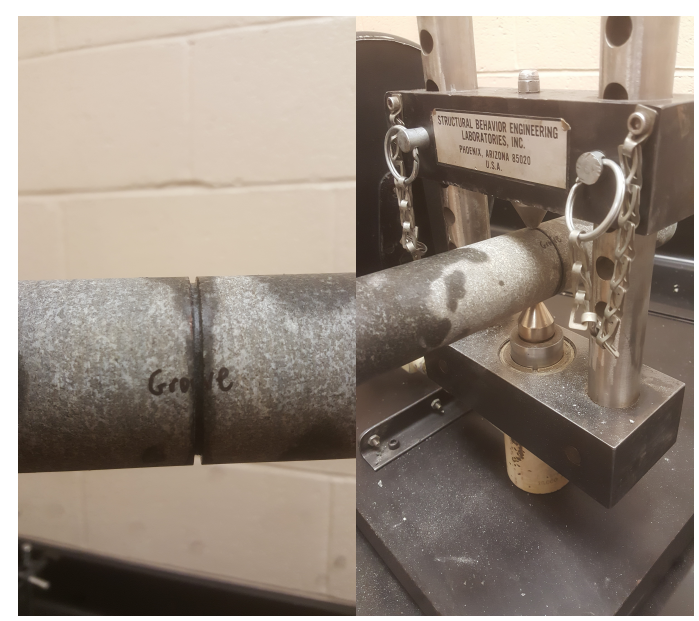

Figure 3.7: Limestone Sample with Groove Cut (L) and Limestone Sample within PLT-10 (R)

applied to the groove and the parent core broke into two pieces with rough surfaces that were parallel to the smooth ends.

The cores were then placed in a PLT-10 point load test meter and loaded to failure. The platens on the PLT-10 were small enough to apply pressure precisely on the groove. This means the failure plane propagated straight through the groove without deviating through other weak points in the body. The failure planes created rough surfaces parallel to a smooth end, thus, the alignment of the transducers was not be an issue. Figure 3.7 shows a parent core with a groove cut into it along with the core placed inside of the PLT- 10 .

The cores were between 8.2 and $15 \mathrm{~cm}$ in length. The cores were then placed in the same test stand as previous regolith tests and experimented with and without coupling gel on both ends. Once that phase of testing was completed, all but one of the cores had their rough ends sawed off and tested once again with and without couplant. The results showed the difference in wave velocity between rough and smooth samples with and without gel couplant.

In the event that a couplant could be created from the regolith, a second roughness testing series was developed. The second roughness series used one core with a rough surface and had a PVC cast attached to its body. The testing procedure was the same as in R4 with particles sized at 1000-2000 $\mu \mathrm{m}$ continuously added on top of the core until a signal was indistinguishable. The purpose of the second series was to compare the results obtained with a rough sample with regolith on the surface to see if the same trendline from R4, which used a smooth sample, could be obtained. Since the acoustic impedance of the limestone regolith and the testing core is similar, the regolith filled in the voids of the rough and distribute wave energy uniformly across the diameter of the sample. 


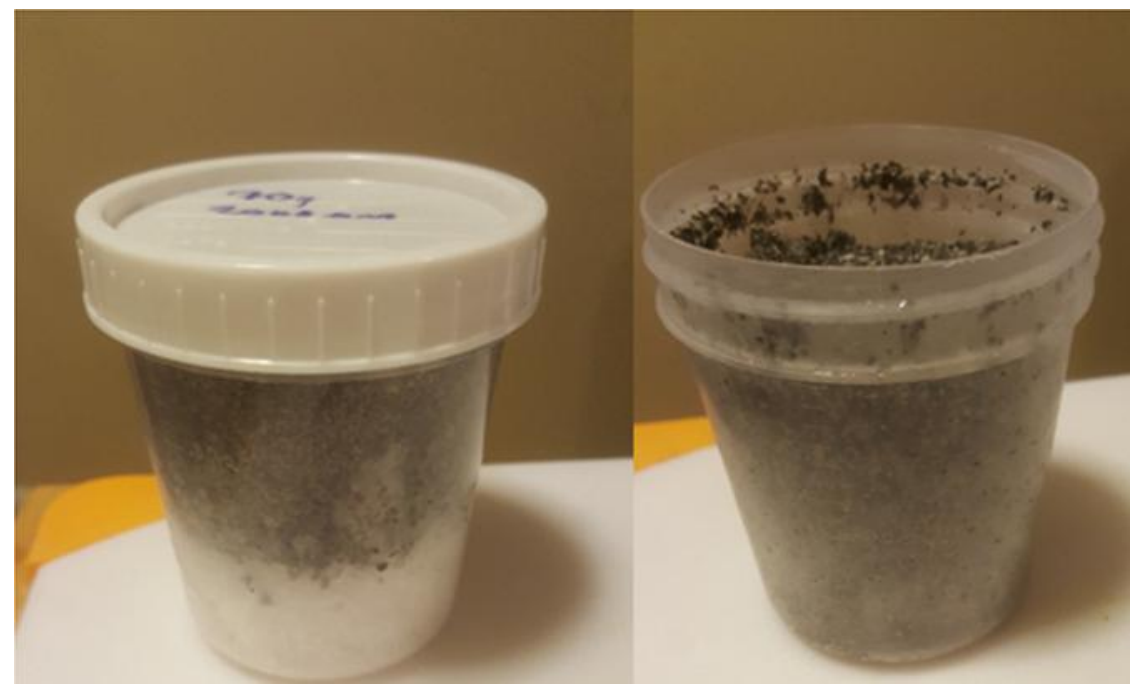

Figure 3.8: Before and After Homogenization

\subsubsection{Ice Testing}

The presence of water in the form of ice on the Moon and Mars, is a primary force behind ISRU efforts. If signal attenuation is affected by material type, then it is conceivable that different concentrations of ice affect wave velocity. Sections 2.1.2 and 2.1.3 have discussed the possibility of water locked into surface regolith that can be used for ISRU efforts. To simulate frozen ice/regolith mixtures, the regolith samples were initially prepared by standard crushing and screening procedures as described in prior sections. Small iced shavings were then prepared using a commercial blender. To ensure complete homogenization of the final mixture, all ice shavings were hand sorted and any pieces larger than 1 centimeter were removed. Next, 100 grams of ice and regolith (at the desired ratio) were combined in the mixing container and shaken up to 2 minutes until completely homogenized. Figure 3.8 shows an example of a blend before and after it is homogenized.

The blend was then placed into a PVC pipe that had an inner diameter of 2 inches and was sealed at the bottom with duct tape. Figure 3.9 is an example of the PVC cast.

The mixture was then gently compressed with a transducer to remove any airpockets. The blend was then placed into a freezer for at least 3 hours before taken out again. Once taken out the freezer, the blend was checked for any surface roughness. If the sample did not have a smooth surface, a transducer was placed on top of the blend and gently rubbed across the surface. The heat transfer from the transducer to the blend melted the ice and created a smooth surface. The sample was then be placed back into the freezer for 15 minutes to refreeze the surface. Prior to testing, the transducers were prepared by using a piece of ice to remove the heat from the 


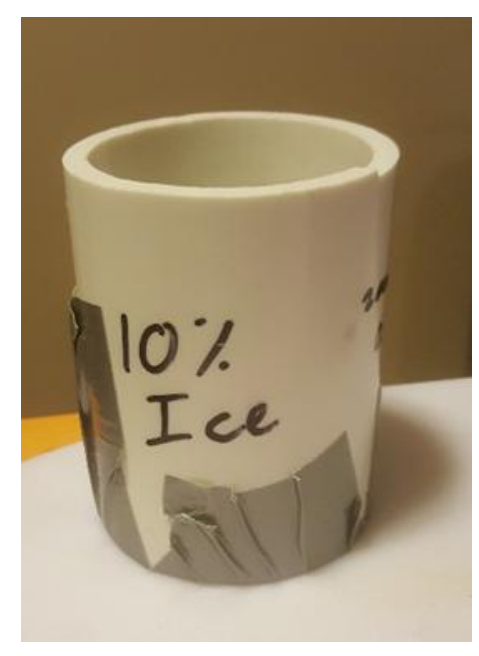

Figure 3.9: PVC Cast

transducer. The heat would otherwise rapidly melt the blend and hinder subsequent measurements. After removing the blend from the freezer, the tape on the bottom of the PVC cast was peeled off, but the blend remained frozen to the sides of the cast. The blend was then placed between both transducers and the distance between the ends of the transducers was measured again. The difference between the new height and the zeroing height would be the height of the sample. Figure 3.10 shows an example of the final setup.

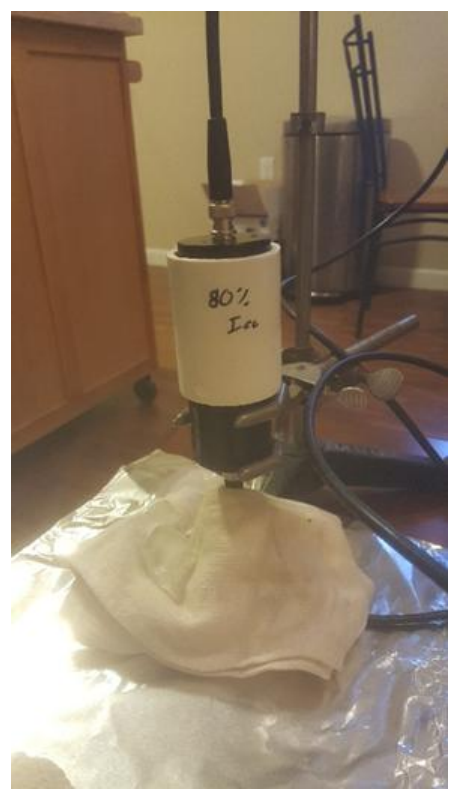

Figure 3.10: Experiment Setup

After the height measurements were completed, ten UPV measurements were conducted on the blend. After the final arrival time was recorded, the height of the 
blend was measured once more. This procedure for the first ice test series used 10002000 micron particles with increasing concentrations of ice at increments of $10 \%$ of the total mass. The second ice series used three of the same particle size distributions as in $\mathrm{R} 2$ with top sizes of 2000 microns: $\mathrm{k}$ values of $0.1,0.5$, and 1.0. The distributions were compared to ice concentrations of 10, 50 , and $90 \%$ and yielded a total of nine samples for testing.

\subsection{Analytical Methods}

\subsubsection{Length Analysis}

Although signal attenuation is different through concrete than it is through limestone or chalk, the minimum number of points necessary to form a sufficient relationship between wave velocity and travel distance has to be established. To analyze the data collected, a MATLAB script was developed that created lines of best fit between every possible combination of data points and estimated the error of the intercepts when they were compared to the baseline velocity. The error between the true velocity and the intercepts was compiled and the average and maximum error with a certain number of points were determined. The maximum error was used for comparison because the average error does not show the range of erroneous values that can be measured.

\subsubsection{Regolith and Roughness Analysis}

To evaluate the signal attenuation through $\mathrm{R} 1$ and $\mathrm{R} 2$ regolith testing series, empirical models and two-dimensional plots were developed from the experimental data. The coefficient of determination $\left(\mathrm{R}^{2}\right)$ and the adjusted coefficient of determination $\left(R_{a d j}^{2}\right)$ were used to validate the accuracy of each model. The significance of each parameter was determined by testing the hypothesis that each parameter did not affect signal attenuation. The analysis of variance and f-statistic values were used to determine the parameter's significance. Parameters with a p-value greater than 0.05 were considered insignificant. The statistical models were created in the statistics program JMP.

To analyze the data from the remainder of the regolith series and the data gathered from the roughness testing, two-dimensional plots were constructed and used the limestone core baseline velocity to observe the change in velocity with respect to distance. In the analysis of fourth and fifth regolith series as well as the second roughness series, a procedure was used to determine if the differences in trendlines were significant. In order to simplify computations, the trendlines were converted into 
linear functions. The next step of the procedure tested the coefficients of determination for both data sets. If the coefficients of determination were above 0.90 then the significance between the data scatterings was determined by an F-test. If the p-value calculated was greater than 0.05 , it meant that both data sets had similar scattering. For tests that measured p-values above 0.05, two more F-tests were conducted on the differences between the slopes and intercepts of the trendlines. If the p-values of the F-tests were greater than 0.05 , it meant that the intercepts or slopes of the trendlines were statistically proven to be similar depending on the parameter being tested. If the p-value was less than 0.05 , it meant the slopes or intercepts were different. Details of the calculations can be reviewed in Napier-Munn (2014).

For a summary test matrix with brief description of each series, see Table 3.5.

Table 3.5: Summary Test Matrix

\begin{tabular}{|c|c|c|c|}
\hline Series & Designation & Description & Parameters \\
\hline 点愛 & L & $\begin{array}{l}\text { Evaluate the difference in wave } \\
\text { velocity across increasing distance in } \\
\text { concrete with and without a couplant }\end{array}$ & 1) Gel Couplant \\
\hline 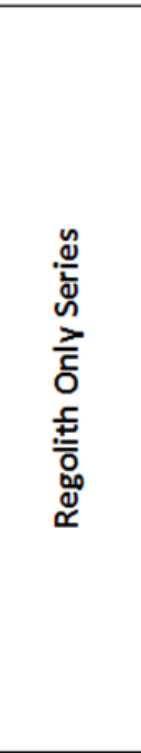 & $\mathrm{R} 2$ & $\begin{array}{l}\text { Evaluation of parameters: which } \\
\text { parameters significantly affect the } \\
\text { velocity and amplitude of ultrasonic } \\
\text { waves? } \\
\text { With a revised procedure, how does } \\
\text { increasing the mass of a blend affect } \\
\text { test results? Also, by using less mass, } \\
\text { will other parameters have a greater } \\
\quad \text { effect? } \\
\text { Using the nine indivdual particle size } \\
\text { fractions (meaning no blends), how } \\
\text { does particle top size affect ultrasonic } \\
\quad \text { waves? } \\
\text { Further testing of three particle size } \\
\text { fractions in order to find the precise } \\
\text { relationship between particle top size } \\
\text { and wave velocity and amplitude. } \\
\text { Using a single particle size class, chalk } \\
\text { regolith was combined with the same } \\
\text { limestone core to evaluate the } \\
\text { difference using different materials. }\end{array}$ & $\begin{array}{l}\text { 1) Particle depth } \\
\text { 2) Particle Size Distribution } \\
\text { 3) Top Particle Size } \\
\text { 4) Compaction Ratio } \\
\text { 1) Blend Mass } \\
\text { 2) Particle Size Distribution } \\
\text { 3) Top Particle Size } \\
\text { 1) Total Mass } \\
\text { 2) Top Particle Size } \\
\text { 1) Total Mass } \\
\text { 2) Top Particle Size } \\
\text { 1) Total Mass } \\
\text { 2) Top Particle Size }\end{array}$ \\
\hline 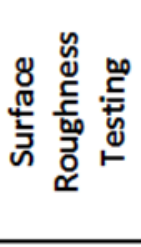 & SR 1 & $\begin{array}{l}\text { Assess the difference between smooth } \\
\text { and rough samples with and without a } \\
\qquad \text { couplant } \\
\text { Further testing of roughness by using } \\
\text { a single particle size class in order to } \\
\text { compare to results found in R4. }\end{array}$ & $\begin{array}{l}\text { 1) Gel Couplant } \\
\text { 1) Particle Top Size } \\
\text { 2) Regolith Mass }\end{array}$ \\
\hline 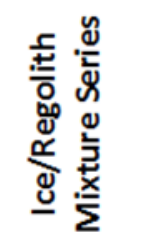 & $1-2$ & $\begin{array}{l}\text { Tests use a combination of ice and a } \\
\text { single particle size fraction at different } \\
\text { ice mass fractions. } \\
\text { See the difference in wave velocity } \\
\text { and amplitude as particle size } \\
\text { distribution changes. }\end{array}$ & $\begin{array}{l}\text { 1) Ice Mass } \\
\text { 1) Ice Mass } \\
\text { 2) Particle Size Distribution }\end{array}$ \\
\hline
\end{tabular}




\section{Chapter 4}

\section{Results and Discussion}

\subsection{Length of Sample}

\subsubsection{Direct and Semi-Direct Testing}

UPV testing on the three long concrete beams $(1,3,6 \mathrm{~m})$ showed a general reduction in wave velocity as a function of test length. Figure 4.1 shows the combination of points generated by direct and semi-direct testing with gel and compared to tests without gel. The red border represents the baseline velocity at $4,845 \mathrm{~m} / \mathrm{s}$, which was derived from testing the concrete cores.

The first major observation from this data is that UPV can successfully transmit wave velocity up to six meters with and without coupling gel; however, the measurements may not be able to accurately represent the actual mechanical properties of the material. In Figure 4.1, P-wave velocity decreases as distance between transducers increases for tests with and without coupling gel, though the wave velocity decreases at a faster rate when coupling gel is not used. The second major observation is that the data generally follows consistent trends, suggesting that a simple correction procedure can predict UPV values commensurate with the baseline. The addition of coupling gel clearly improved the consistency of wave velocity measurements especially at lengths greater than 3.5 meters. Without gel couplant, the data collected after 3.5 meters behaved erratically and did not follow a general trend.

While signal attenuation is an exponential relationship, these curves appear to be on a linear portion of the curve, suggesting that simple regression lines can be used to predict the baseline velocity. The regression lines were extrapolated to zero length, and provided values that were less than one percent different from the baseline. These corrected values indicate what the wave velocity would be without any signal attenuation. 


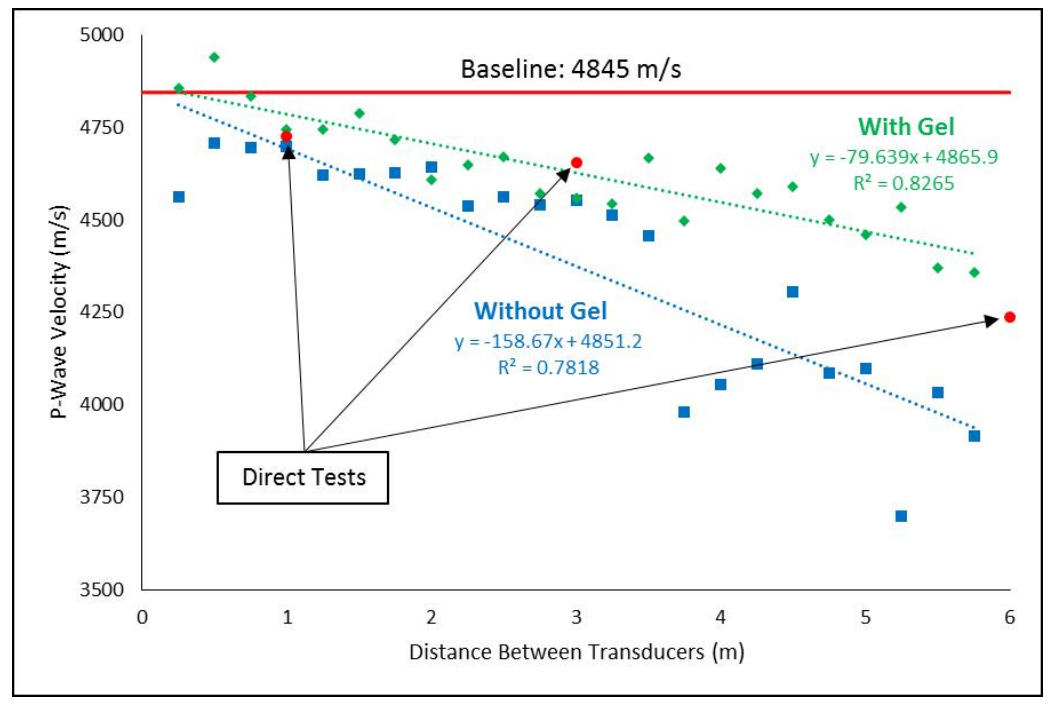

Figure 4.1: Semi-Direct Testing of 6 meter Beam Compared to Direct Testing of 1,3 , and 6 meter Beam

\subsubsection{Error Analysis}

While the the data above shows that that simple linear regression of UPV vs. length can predict baseline values, the accuracy of those predictions was primarily based on the large number of points used in the analysis. The trendlines for each data set included at least 20 points, and as a result, the trendlines smoothed the error in each individual measurement. To further investigate the accuracy of the correction protocol and determine the minimum number of points needed to produce a consistent trendline, the data in Figure 4.1 was analyzed further. In this procedure numerous trendlines were generated using all possible combinations of points. For example, since the no-gel data set contains 23 points, 276 distinct trendlines can be generated by using only two of the data points. Likewise 2,024 trendlines can be generated from 3 points, 10,626 from four points, etc. In total, this data set can produce $16,777,190$ distinct trendlines.

After generating all trendlines, each was extrapolated to zero length to determine the corrected measurement and these values were then compared to the baseline value of $4,845 \mathrm{~m} / \mathrm{s}$. The max error in the corrected value is plotted against the number of points used to create the trendline in Figure 4.2 while Figure 4.3 plots all corrected values collected into boxplots.

Figures 4.2 and 4.3 show that 4 points are sufficient for gel testing since the reductions in maximum error are less significant with only a $13.6 \%$ maximum error but to reduce the amount of maximum error below 5\%,10 points are needed. The figures also show that the change in maximum error without the use of gel couplant slows after 6 points but the maximum error is $27.8 \%$ and 16 points are required to 


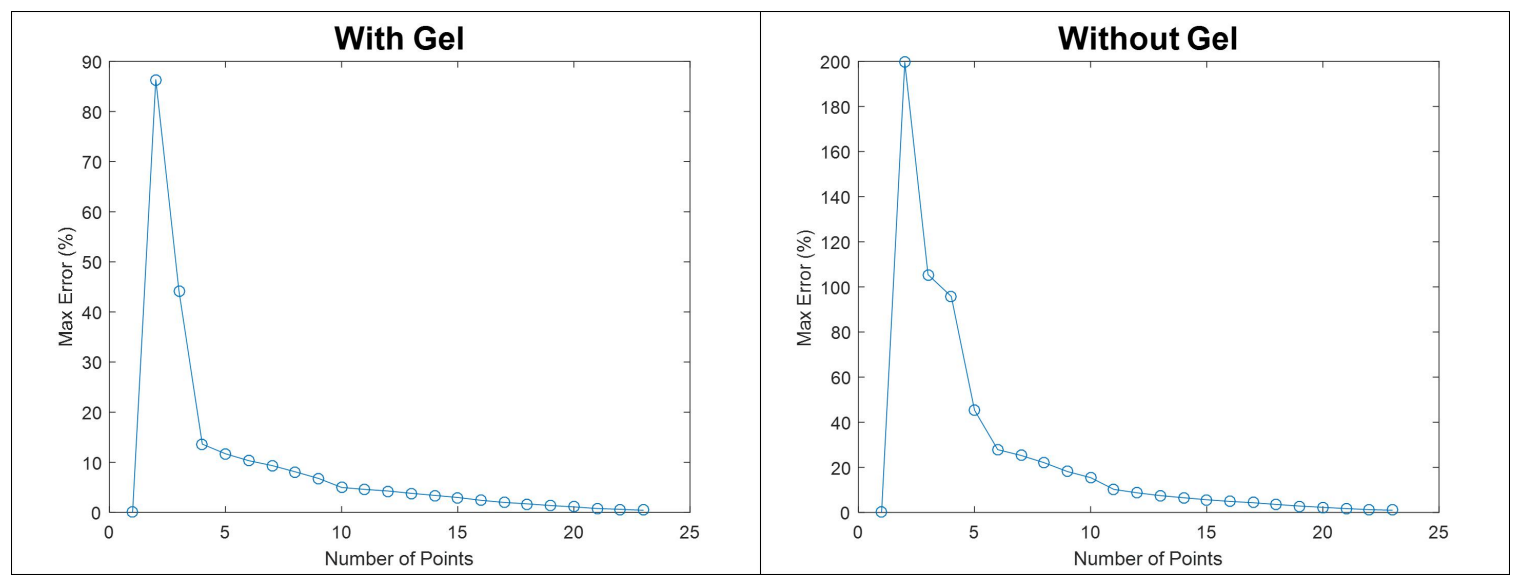

Figure 4.2: Maximum Error of Corrected Values from Baseline Velocity With Gel Couplant (L) and Without Couplant (R)

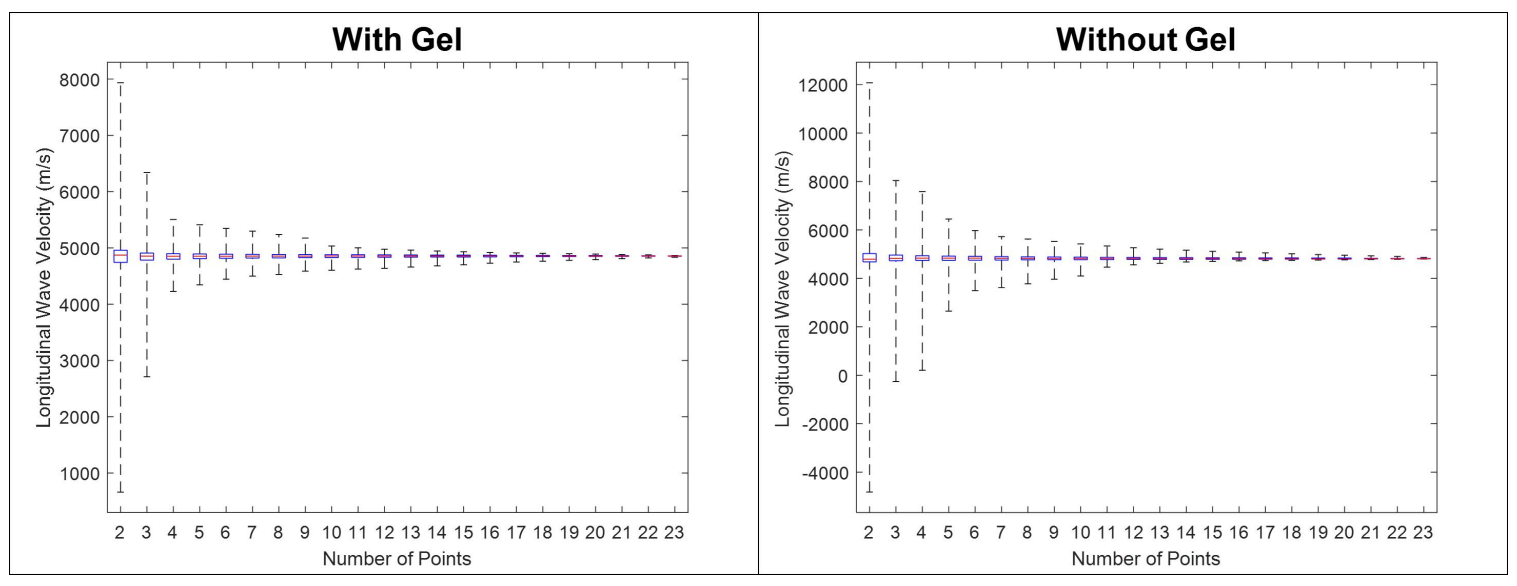

Figure 4.3: Box-Plot of All Corrected Values With Gel Couplant (L) and Without Gel Couplant(R) 
reduce the error below 5\%. The number of points would also fluctuate depending on the amount of void spaces within the sample as well; however, the data shows that even without the use of a couplant, a trendline can be formed with a small amount of error between the target truth value and the corrected value from the trendline. Although these same lines will not be seen when testing a different material, the methodology will remain the same.

\subsection{Regolith Testing}

\subsubsection{Statistical Analysis of R1}

In total, five "regolith" test series were performed to determine the influence of regolith cover on UPV response. In addition, these test series determine the regolith characteristics that most influenced signal attenuation and methods to correct these measurements. The goal of the first regolith series was to test the significance between the four testing parameters: particle top, particle size distribution, compaction, and depth. Out of the 27 tests conducted, 6 tests did not produce a distinguishable signal namely $1,7,12,14,22$, and 24 . All of the tests that did not produce a measurement had top sizes larger than 500 microns indicating that at certain top size, signal acquisition may not be possible regardless of the other parameters. Simple linear regression models for both responses were made with each parameter to identify relationships between the parameters and the responses. The base ten logarithm had to be taken for amplitude responses since the range of values differed by orders of magnitude. The results of the regolith test series are shown in Table 4.1

Based on the results shown in Table 4.1, depth of regolith has a significant effect on the velocity, while other parameters are not as influential to wave velocity. The maximum velocity produced was $2270 \mathrm{~m} / \mathrm{s}$ at the smallest depth of $1 \mathrm{~cm}$. For increasing depths of regolith, relative velocity decreases by up to $70 \%$ between the greatest velocity at $1 \mathrm{~cm}$ depth and the slowest velocity at $3 \mathrm{~cm}$.

Additionally, particle size distribution seems to affect the amplitude of a signal more than any other parameter. Tests that had the widest size distribution modulus (0.1) produced the greatest amplitudes. As the size distribution narrows, the relative $\log _{10}$ (amplitude) decreases by up to $44 \%$ between the greatest $\log _{10}$ (amplitude) with a $\mathrm{k}$ value of 0.1 and the slowest $\log _{10}$ (amplitude) with a $\mathrm{k}$ value at 1.0 .

The results show that blends with the shallowest depths and widest distributions produce the greatest velocities and amplitudes; however, the figures indicate that smaller top size and greater compaction ratios influence wave velocity and amplitude to some degree.

To evaluate the statistical significance of combining the parameters for both the 
Table 4.1: First Regolith Testing Series Results

\begin{tabular}{|c|c|c|c|c|c|c|}
\hline Test \# & $\begin{array}{c}\text { Top Size } \\
(\mu \mathrm{m})\end{array}$ & $\begin{array}{l}\text { Size Distr. } \\
\text { Modulus }\end{array}$ & Compaction & $\begin{array}{c}\text { Regolith } \\
\text { Depth (cm) }\end{array}$ & $\begin{array}{c}\text { P-Wave } \\
\text { Velocity }(\mathrm{m} / \mathrm{s})\end{array}$ & Log(Amplitude) \\
\hline 1 & 2000 & 0.5 & $\operatorname{Max}$ & 2 & \multicolumn{2}{|c|}{ NO SIGNAL } \\
\hline 2 & 500 & 0.5 & $\operatorname{Max}$ & 2 & 1468 & 3.55 \\
\hline 3 & 1000 & 0.5 & $\operatorname{Max}$ & 1 & 2272 & 3.29 \\
\hline 4 & 500 & 0.1 & Some & 2 & 1310 & 4.21 \\
\hline 5 & 1000 & 0.5 & None & 1 & 1835 & 3.03 \\
\hline 6 & 1000 & 0.1 & None & 2 & 1003 & 3.28 \\
\hline 7 & 2000 & 0.5 & Some & 3 & \multicolumn{2}{|c|}{ NO SIGNAL } \\
\hline 8 & 500 & 0.5 & Some & 1 & 1903 & 3.79 \\
\hline 9 & 2000 & 0.1 & Some & 2 & 1333 & 3.89 \\
\hline 10 & 500 & 0.5 & Some & 3 & 783 & 3.14 \\
\hline 11 & 1000 & 0.1 & Some & 3 & 1039 & 4.04 \\
\hline 12 & 1000 & 1 & Some & 3 & \multicolumn{2}{|c|}{ NO SIGNAL } \\
\hline 13 & 1000 & 0.5 & None & 3 & 682 & 2.77 \\
\hline 14 & 1000 & 1 & Max & 2 & \multicolumn{2}{|c|}{ NO SIGNAL } \\
\hline 15 & 1000 & 0.5 & Some & 2 & 975 & 3.16 \\
\hline 16 & 1000 & 0.1 & Some & 1 & 2207 & 4.81 \\
\hline 17 & 1000 & 1 & None & 2 & 801 & 2.69 \\
\hline 18 & 500 & 0.5 & None & 2 & 1017 & 3.34 \\
\hline 19 & 1000 & 0.5 & $\operatorname{Max}$ & 3 & 831 & 3.00 \\
\hline 20 & 1000 & 0.5 & Some & 2 & 991 & 2.98 \\
\hline 21 & 1000 & 0.1 & Max & 2 & 1463 & 4.69 \\
\hline 22 & 2000 & 0.5 & None & 2 & \multicolumn{2}{|c|}{ NO SIGNAL } \\
\hline 23 & 1000 & 1 & Some & 1 & 2064 & 2.91 \\
\hline 24 & 2000 & 0.5 & Some & 1 & \multicolumn{2}{|c|}{ NO SIGNAL } \\
\hline 25 & 500 & 1 & Some & 2 & 1081 & 3.05 \\
\hline 26 & 2000 & 1 & Some & 2 & 1101 & 2.82 \\
\hline 27 & 1000 & 0.5 & Some & 2 & 941 & 3.10 \\
\hline
\end{tabular}




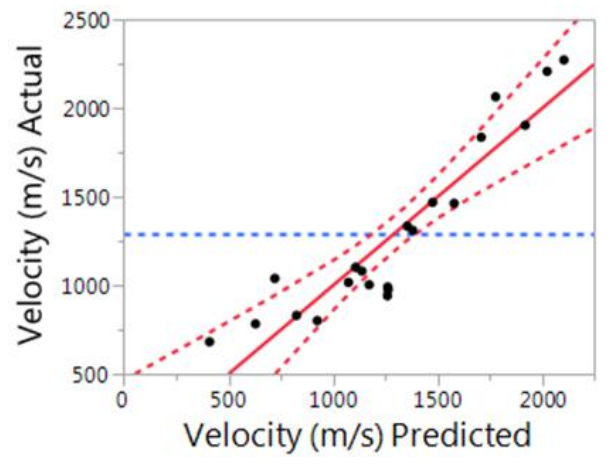

Figure 4.4: First Regolith Series Velocity Prediction Model

Table 4.2: First Regolith Series Velocity Statistical Analysis

\begin{tabular}{|l|cccccc|}
\hline Parameter & $\begin{array}{c}\text { Sum of } \\
\text { Squares }\end{array}$ & df & $\begin{array}{c}\text { Mean } \\
\text { Square }\end{array}$ & F-value & $\begin{array}{c}\text { P-Value } \\
\text { Prob }>\text { F })\end{array}$ & Significant \\
\hline Top Size (um) & $1.34 \mathrm{E}+03$ & 1 & $1.34 \mathrm{E}+03$ & 0.03 & 0.8622 & No \\
Size Distribution & $1.39 \mathrm{E}+05$ & 1 & $1.39 \mathrm{E}+05$ & 3.24 & 0.0909 & No \\
Compaction Ratio & $3.69 \mathrm{E}+05$ & 1 & $3.69 \mathrm{E}+05$ & 8.6 & 0.0098 & Yes \\
Depth (mm) & $3.68 \mathrm{E}+06$ & 1 & $3.68 \mathrm{E}+06$ & 85.79 & $<0.0001$ & Yes \\
Model & $4.14 \mathrm{E}+06$ & 4 & $1.04 \mathrm{E}+06$ & 24.124 & $<0.0001$ & Yes \\
& & & & & & \\
R-Squared & 0.86 & Adj. R-Squared & 0.82 & & \\
RMSE & 207.14 & & & & \\
\hline
\end{tabular}

velocity and amplitude models, multiple linear regression was used to determine the most influential parameters in the final prediction of velocity and amplitude. Figure 4.4 and Figure 4.5 are plots of actual versus predicted values for velocity and amplitude that were derived from the multiple linear regression analysis and Tables 4.2 and 4.3 contain the F-test parameters that test the significance of the models. The null hypothesis for each F-test is that each model term is insignificant in the prediction. A significance level of 0.05 was used as the criteria for rejecting the null hypothesis, and as such a Prob $>$ F value less than 0.05 indicates that the model term is significant.

For the velocity analysis in Figure 4.4 and Table 4.2, a relatively strong prediction is obtained using depth, compaction ratio, and particle size distribution as variables. Regolith depth has the strongest influence on $\mathrm{P}$-wave velocity while compaction ratio is the next most influential parameter. 


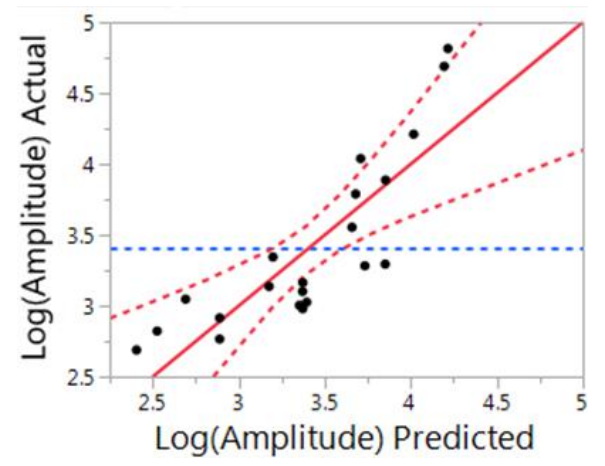

Figure 4.5: First Regolith Series Amplitude Prediction Model

Table 4.3: First Regolith Series Amplitude Statistical Analysis

\begin{tabular}{|lcccccc|}
\hline Parameter & $\begin{array}{c}\text { Sum of } \\
\text { Squares }\end{array}$ & df & $\begin{array}{c}\text { Mean } \\
\text { Square }\end{array}$ & F value & $\begin{array}{c}\text { P-Value } \\
\text { Prob > F) }\end{array}$ & Significant \\
\hline Top Size (um) & 0.04 & 1 & 0.04 & 0.311 & 0.5847 & No \\
Size Distribution & 4.11 & 1 & 4.11 & 30.56 & $<0.0001$ & Yes \\
Compaction Ratio & 0.47 & 1 & 0.47 & 3.5 & 0.08 & No \\
Depth (mm) & 0.56 & 1 & 0.56 & 4.16 & 0.582 & No \\
Model & 5.31 & 4 & 1.33 & 9.87 & 0.0003 & Yes \\
& & & & & & \\
R-Squared & 0.71 & Adj. R-Squared & 0.64 & & \\
RMSE & 0.37 & & & & \\
\hline
\end{tabular}

The amplitude analysis in Figure 4.5 and Table 4.3 indicates a weaker correlation than for the velocity analysis, but it still indicates a relationship between amplitude and certain testing parameters. Of these parameters, size distribution is the most significant.

Size distribution affects both the velocity and amplitude of a signal. Greater velocities and amplitudes have wider particle distributions. Blends with a narrow particle size distribution result in an increase of void spaces between particles, since smaller particles are not available to occupy the void spaces. For blends with a wide size distribution, the variety in particle sizes allow for void spaces to be filled more readily, which produces more particles interfaces for waves to propagate along.

Compaction ratio affects both velocity and amplitude because it determines particle density by reducing void spaces between particles. Increased compaction reduces the amount of voids, and benefits wave velocity. Regardless of size distribution, increased compaction of regolith blends with either narrow or wide size distributions increases wave velocity and amplitude. A greater increase in responses; however, results from greater compaction of blends with a wider size distribution since fewer void spaces are present than in a narrowly sized blend. 
Regolith depth specifies the total amount of particles and voids a wave has to pass through. As the depth increases, the amount of particles and void spaces increase as well. This means that the energy of the wave decreases as it propagates between the increasing number of particles and voids. The loss of energy results in both a loss in signal amplitude and slower velocity although it has greater statistical significance to wave velocity.

Top size is not statistically significant in either analysis; however, the lack of significance in the analyses does not indicate its influence on signal transmission since 6 tests with large top sizes were discarded from the analysis. It is reasonable to believe that at a certain top size, a signal cannot be obtained but once a signal is received, the top size quickly loses its significance. The other parameters that specify the amount of the maximum particle size such as depth and particle size distribution likely influence the signal's reception as well. This maximum top size is likely between $1-2 \mathrm{~mm}$ but it may change depending on the other variables.

The most notable observation from this test series is that not a single test produced a velocity comparable to the baseline. The closest velocity was still $60 \%$ slower than the baseline. Regolith can still be used as a couplant, but perhaps with shallower depths and particle sizes should be used to determine limiting values for these parameters that will produce distinguishable transmissions and velocities that are more representative of the material being tested.

\subsubsection{Procedure Revisions and R2 Statistical Analysis}

Due to the results in the previous series, the procedure for second regolith series was be revised to identify the particle top size limit and change in velocity according to regolith depth. The new procedure removed compaction ratio as a variable instead maximum compaction was used in order to maximize the energy efficiency of the wave. A specified mass of regolith took the place of a specific depth. The new regolith masses also corresponded to shallower depths than what was tested before. The additional benefits of this new procedure decreased testing time and limited sources of human error since depth only had to be measured once. Instead of a Box-Behnken experimental design, a full factorial design was used. Using these values described in Section 3.3.4, two multiple regression models were developed and F-tests evaluated the significance of the models. The same null hypotheses from the previous analyses were used and a signifcance level of 0.05 was implemented. Table 4.4 shows the raw data from the second regolith series with Figures 4.6 and 4.7 showing the multiple linear regression models of wave velocity and amplitude respectively. Tables 4.5 and 4.6 contain the F-test parameters for wave velocity and amplitude respectively. 
Table 4.4: Second Regolith Testing Series Results

\begin{tabular}{|c|c|c|c|c|c|c|}
\hline Test \# & $\begin{array}{c}\text { Top Size } \\
\quad(\mu \mathrm{m})\end{array}$ & $\begin{array}{l}\text { Size Distr. } \\
\text { Modulus }\end{array}$ & $\begin{array}{l}\text { Regoltih } \\
\text { Mass (g) }\end{array}$ & $\begin{array}{l}\text { Depth } \\
(\mathrm{mm})\end{array}$ & $\begin{array}{c}\text { P-Wave } \\
\text { Velocity }(\mathrm{m} / \mathrm{s})\end{array}$ & Log(Amplitude) \\
\hline 1 & 500 & 0.1 & 15 & 3 & 3704 & 10.96 \\
\hline 2 & 500 & 0.1 & 30 & 8 & 2764 & 11.05 \\
\hline 3 & 500 & 0.1 & 45 & 13 & 2078 & 10.60 \\
\hline 4 & 500 & 0.5 & 15 & 2 & 4015 & 10.90 \\
\hline 5 & 500 & 0.5 & 30 & 6 & 2615 & 9.80 \\
\hline 6 & 500 & 0.5 & 45 & 11 & 1962 & 9.20 \\
\hline 7 & 500 & 1 & 15 & 2 & 3888 & 9.81 \\
\hline 8 & 500 & 1 & 30 & 7 & 2649 & 8.81 \\
\hline 9 & 500 & 1 & 45 & 11 & 1801 & 6.77 \\
\hline 10 & 1000 & 0.1 & 15 & 2 & 3968 & 10.21 \\
\hline 11 & 1000 & 0.1 & 30 & 6 & 2717 & 10.20 \\
\hline 12 & 1000 & 0.1 & 45 & 11 & 2017 & 9.52 \\
\hline 13 & 1000 & 0.5 & 15 & 3 & 3932 & 9.88 \\
\hline 14 & 1000 & 0.5 & 30 & 6 & 2668 & 7.59 \\
\hline 15 & 1000 & 0.5 & 45 & 11 & 1911 & 6.78 \\
\hline 16 & 1000 & 1 & 15 & 4 & 4365 & 10.23 \\
\hline 17 & 1000 & 1 & 30 & 7 & 2653 & 8.16 \\
\hline 18 & 1000 & 1 & 45 & 10 & 2021 & 6.55 \\
\hline 19 & 2000 & 0.1 & 15 & 4.1 & 3994 & 11.09 \\
\hline 20 & 2000 & 0.1 & 30 & 7.61 & 2902 & 11.06 \\
\hline 21 & 2000 & 0.1 & 45 & 11.87 & 2144 & 9.30 \\
\hline 22 & 2000 & 0.5 & 15 & 4.78 & 4398 & 9.41 \\
\hline 23 & 2000 & 0.5 & 30 & 8.06 & 3293 & 8.80 \\
\hline 24 & 2000 & 0.5 & 45 & 12.49 & 2162 & 7.31 \\
\hline 25 & 2000 & 1 & 15 & 3.85 & 4154 & 9.75 \\
\hline 26 & 2000 & 1 & 30 & 8.68 & 2904 & 7.40 \\
\hline 27 & 2000 & 1 & 45 & 11.86 & 2213 & 6.83 \\
\hline
\end{tabular}




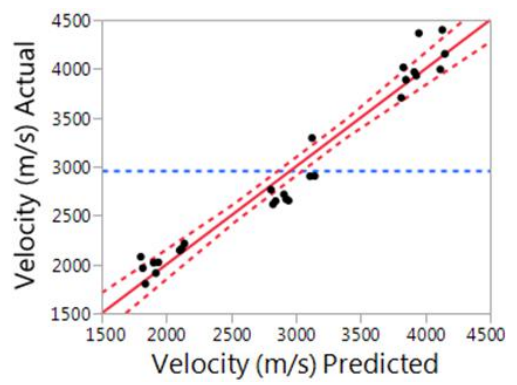

Figure 4.6: Second Regolith Series Velocity Prediction Model

Table 4.5: Second Regolith Series Velocity Statistical Analysis

\begin{tabular}{|ccccccc|}
\hline Parameter & $\begin{array}{c}\text { Sum of } \\
\text { Squares }\end{array}$ & df & $\begin{array}{c}\text { Mean } \\
\text { Square }\end{array}$ & F value & $\begin{array}{c}\text { P-Value } \\
\text { Prob }>\text { F })\end{array}$ & Significant \\
\hline Top Size (um) & $1.34 \mathrm{E}+03$ & 1 & $1.34 \mathrm{E}+03$ & 11.6736 & 0.0024 & Yes \\
Size Distribution & $4.24 \mathrm{E}+05$ & 1 & $4.24 \mathrm{E}+05$ & 0.1578 & 0.6949 & No \\
Mass (g) & $5.74 \mathrm{E}+03$ & 1 & $5.74 \mathrm{E}+03$ & 501.2 & $<0.0001$ & Yes \\
Model & $1.86 \mathrm{E}+07$ & 3 & $6.22 \mathrm{E}+06$ & 171.01 & $<0.0001$ & Yes \\
& & & & & & \\
R-Squared & 0.96 & & Adj. R-Squared & 0.95 & & \\
RMSE & 190.66 & & & & \\
\hline
\end{tabular}

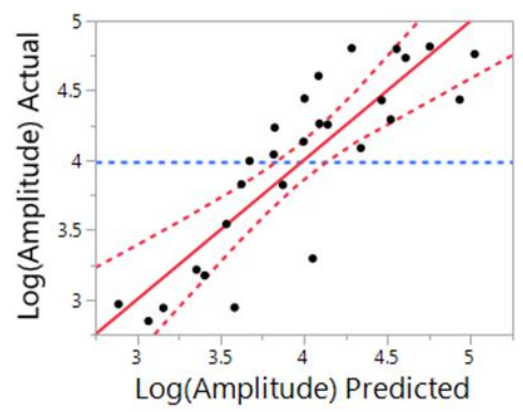

Figure 4.7: Second Regolith Series Amplitude Prediction Model

Table 4.6: Second Regolith Series Amplitude Analysis

\begin{tabular}{|ccccccc|}
\hline Parameter & $\begin{array}{c}\text { Sum of } \\
\text { Squares }\end{array}$ & df & $\begin{array}{c}\text { Mean } \\
\text { Square }\end{array}$ & F value & $\begin{array}{c}\text { P-Value } \\
(\text { Prob }>\text { F })\end{array}$ & Significant \\
\hline Top Size (um) & 0.34 & 1 & 0.34 & 2.74 & 0.1112 & No \\
Size Distribution & 3.9 & 1 & 3.9 & 31.79 & $<0.0001$ & Yes \\
Mass (g) & 3.94 & 1 & 3.94 & 32.05 & $<0.0001$ & Yes \\
Model & 8.18 & 3 & 2.73 & 22.2 & $<0.0001$ & Yes \\
& & & & & & \\
R-Squared & 0.74 & & Adj. R-Squared & 0.71 & & \\
RMSE & 0.35 & & & &
\end{tabular}




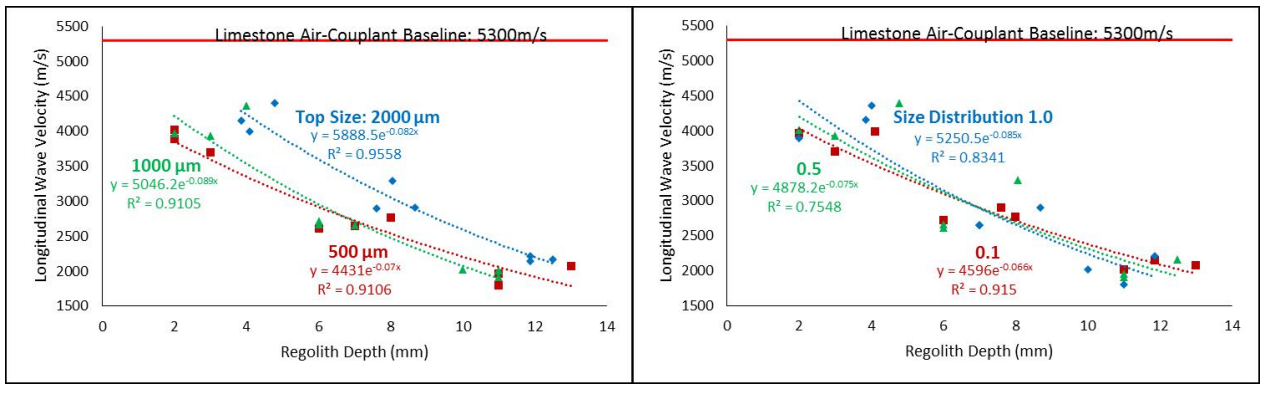

Figure 4.8: Regolith Depth vs. Longitudinal Wave Velocity Sorted by Particle Top Size (L) and Size Distribution (R)

Table 4.5 shows that the particle top size greatly increases in significance to wave velocity from the previous statistical analysis. Top size also increased in significance to wave amplitude when compared to the amplitude statistical analysis in the previous section. Size distribution is not a significant influence on wave velocity; however, it is influential on wave amplitude as shown in Table 4.6. The coefficients of determination of both models increased from the previous analyses indicating stronger relationships between the response variables and the testing parameters. The results from the statistical models are corroborated by comparing regolith depth to the respective response variable when the data is grouped by top size or size distribution. Figures 4.8 shows the relationship between regoltih depth and wave velocity when sorted by particle top size and size distribution. The data was fit with exponential curves to corroborate with the fundamental notion that signal attenuation is an exponential phenomenon.

From Figure 4.8, as particle top size increases and as size distribution narrows, wave velocities increase. Interestingly, these data seem to indicate that extrapolation to zero depth prodcues a value similar to the baseline. Altogether, the influence of particle size is now noticeable when smaller amounts of regolith impede the path of the wave. The data from the test series suggests that a correction procedure utilizing regolith depth and particle size can be used to calculate corrected velocities. The next three regolith testing series attempt to validate and develop the procedure.

\subsubsection{Isolating the Influence of Particle Size}

To isolate the influence of top size with increasing regolith depth, the third regolith series utilized the individual size fractions from blends previously used, and meant particle size distribution was discarded as a parameter. Figure 4.9 shows the results of the tests on each particle size class with an exponential relationship between depth and wave velocity displayed. Figure 4.10 plots the corrected value for each top size in a bar graph. 

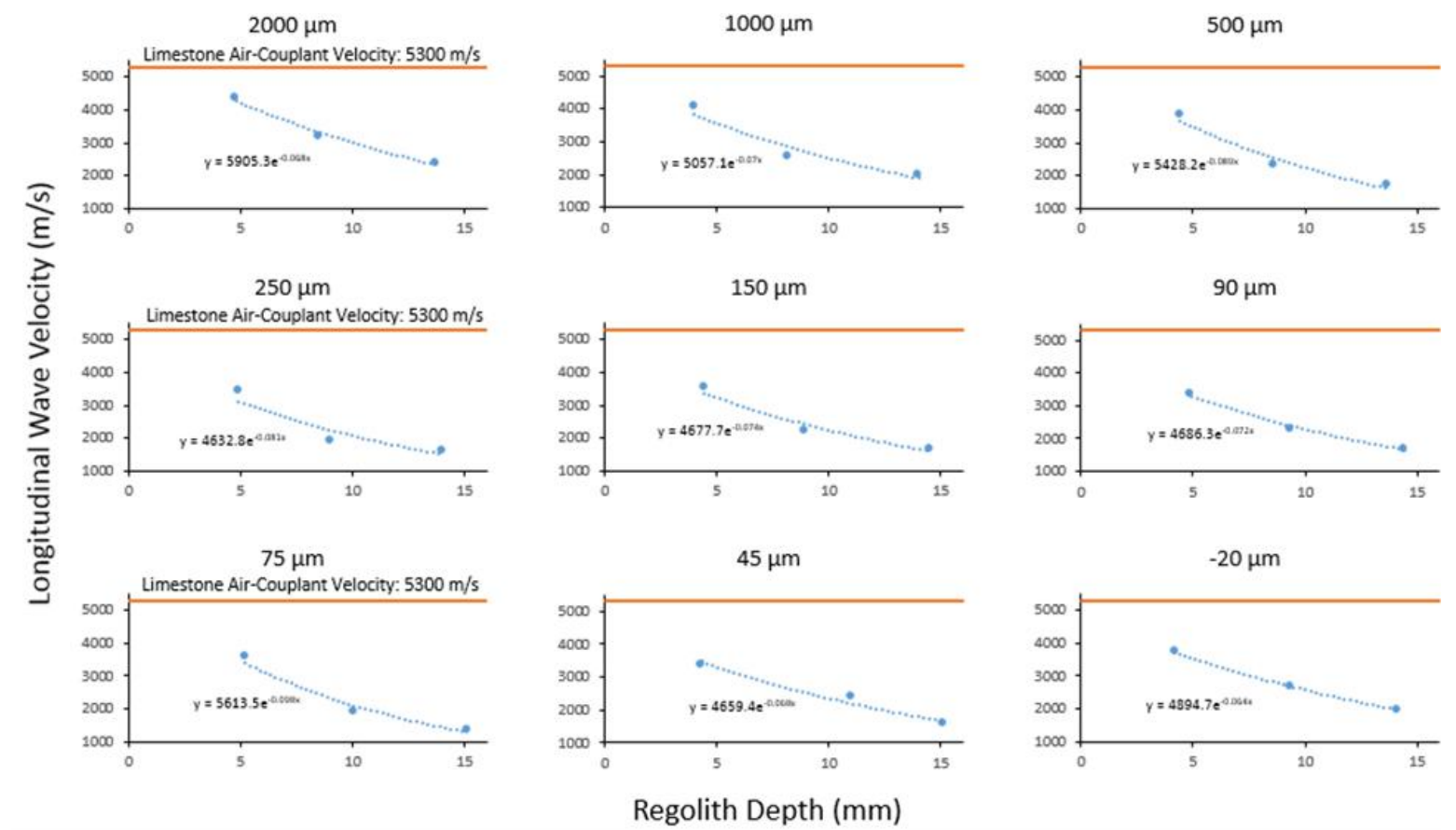

Figure 4.9: Regolith Depth vs. Longitudinal Wave Velocity Across All Size Classes

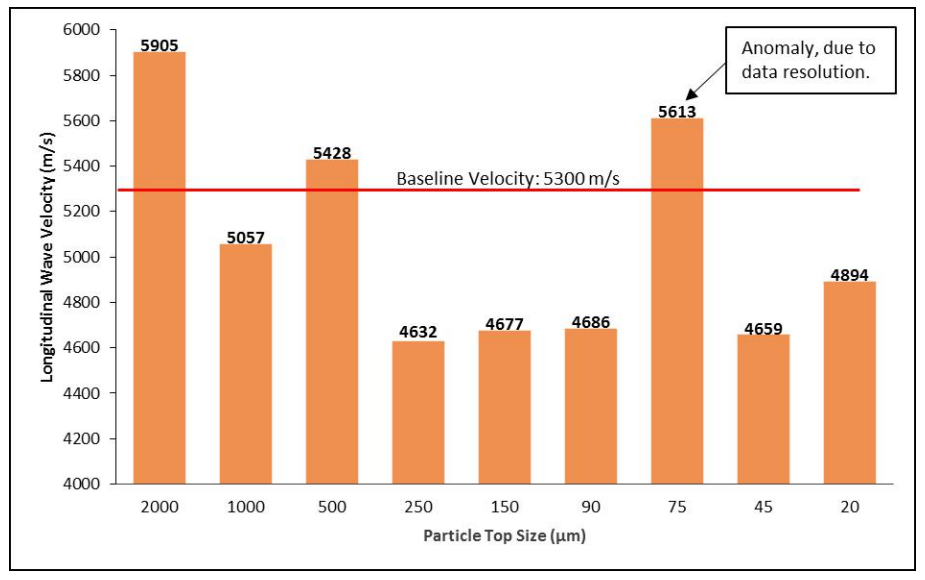

Figure 4.10: Corrected Wave Velocity for Each Size Classes 
These plots of measured wave velocity versus depth show a nearly exponential trend for all top sizes. By back extrapolating this trendline to a depth of zero, the value is able to approximate the baseline velocity. Figure 4.10 shows that as top size increases the wave velocty also increases (with the exception at 75 microns); however, the data does not provide sufficient resolution. Although three points is the minimum required to create a regression line, more points are required to reduce the the amount of error.

\section{Enhancing Data Resolution}

To provide additional resolution to the relationship between regolith depth and wave velocity, two additional test series were conducted using a broader range of regolith depth at a finer increment. The results of the fourth test series are shown in Figure 4.11 and shown as linear function with their natural log of wave velocity in Figure 4.12. In this test, an increasing amount of regolith was layered on top of a single limestone core at increments of 5 grams with 10 UPV measurements taken after each increment was added. Three different top sizes (2000, 500, and 45 microns) were tested, and once again wave velocity was recorded as a function of depth.

Data from the graphs clearly show an exponential decay in measured wave velocity with increasing regolith depth. Additionally, extrapolation of these curves to zero regolith depth provide good estimates of the baseline $\mathrm{P}$-wave velocity with the largest size class of 2,000 microns producing the best estimate of wave velocity. From the figures, a larger particle size has a lesser effect on wave velocity; however, each line shares nearly the same slope indicating that the signal attenuation is the same between all size classes. In order to compare the trendlines, the three sets of data were tested using a combination of F-tests to determine if their intercepts, slopes, and data scattering were statistically similar using the procedure from Napier-Munn (2014). The null hypothesis for each F-test is that each model term is insignificant in the prediction. A significance level of 0.05 was used as the criteria for rejecting the null hypothesis, and as such a Prob $>F$ value less than 0.05 indicates that the model term is significant. The results are displayed in Table 4.7.

From Table 4.7, the comparison between trendlines shows that slopes and data scattering are similar to each other. The comparisons demonstrate that material properties of the regolith will influence wave velocity; although, none of the lines produced statistically significant comparisons between intercepts. The statistical difference indicates that the estimations for baseline velocity are dependent on the top size of the regolith. 


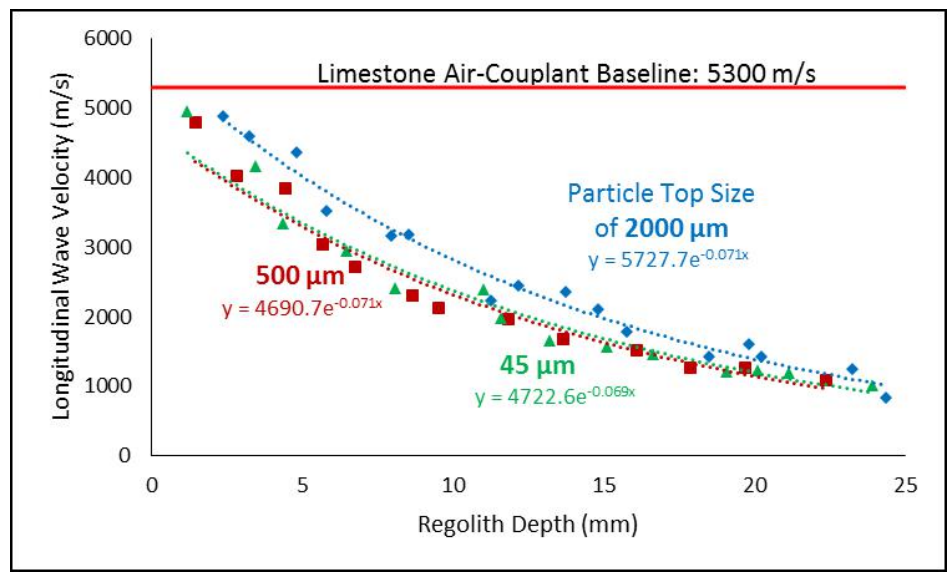

Figure 4.11: Regolith Depth vs. Longitudinal Wave Velocity Across Three Size Classes

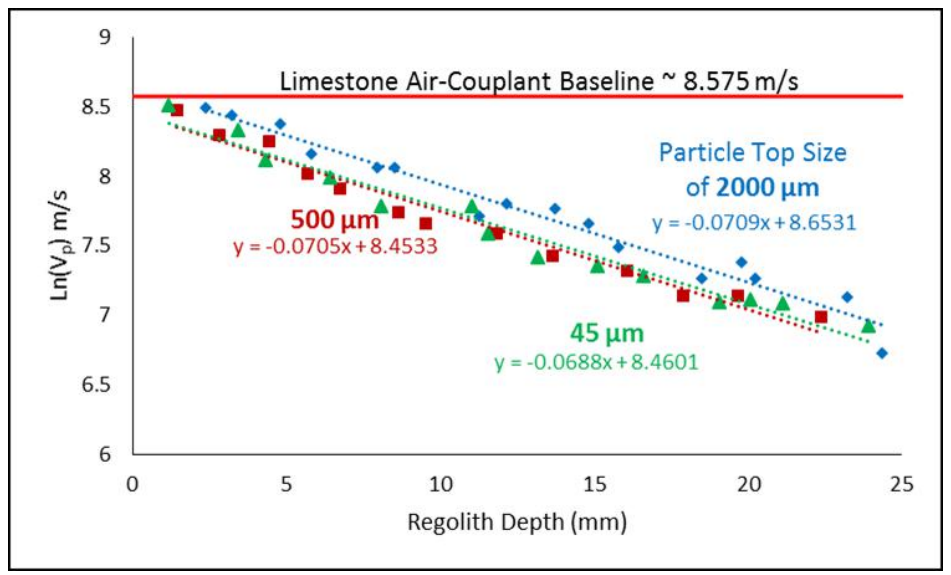

Figure 4.12: Linearized Regolith Depth vs. Longitudinal Wave Velocity Across Three Size Classes

Table 4.7: Comparison Between Trendlines

\begin{tabular}{|l|cc|cc|cc|}
\hline \multirow{2}{*}{ Series Comparison } & \multicolumn{2}{|c|}{ Scatter } & \multicolumn{2}{c|}{ Slope } & \multicolumn{2}{c|}{ Intercept } \\
\cline { 2 - 7 } & $\mathrm{P}(\mathrm{F})$ & $\begin{array}{c}\text { Statistically } \\
\text { Different }\end{array}$ & $\mathrm{P}(\mathrm{F})$ & $\begin{array}{c}\text { Statistically } \\
\text { Different }\end{array}$ & $\mathrm{P}(\mathrm{F})$ & $\begin{array}{c}\text { Statistically } \\
\text { Different }\end{array}$ \\
\hline $2000 \mu \mathrm{m}$ vs $500 \mu \mathrm{m}$ & 0.4091 & No & 0.9467 & No & 0.000 & Yes \\
$2000 \mu \mathrm{m}$ vs $45 \mu \mathrm{m}$ & 0.4706 & No & 0.6782 & No & 0.000 & Yes \\
$500 \mu \mathrm{m}$ vs $45 \mu \mathrm{m}$ & 0.4394 & No & 0.7411 & No & 0.000 & Yes \\
\hline
\end{tabular}

*Note: Critical value of 0.05 used to determine significance 


\section{Evaluating Difference in Regolith Material}

All previous test series were conducting using limestone core material and limestone regolith material that was produced from the same original sample lot. To identify any disparities due to dissimilar material types, additional testing was conducted using regolith produced from an Austin chalk sample. The test procedure was identical to those of the limestone regolith testing with the only difference being regolith material. The data from this test is shown in Figure 4.13 with the natural $\log$ of the results shown in Figure 4.14. Table 4.8 shows the results from comparing the two trendlines using the same F-test from the previous results.

Although the intercepts of lines provide good estimates of the baseline velocity, the slopes are statistically different from one another, which weakens the capability of the regression lines to predict the baseline velocity. The difference in slopes confirms that a different regolith material from the body underneath will affect signal attenuation differently as opposed to regolith of the same material type. The ultrasonic waves will travel at different velocities when moving through each material and attenuate at different rates as opposed to traveling through the same material that comprises the regolith and rock mass underneath.

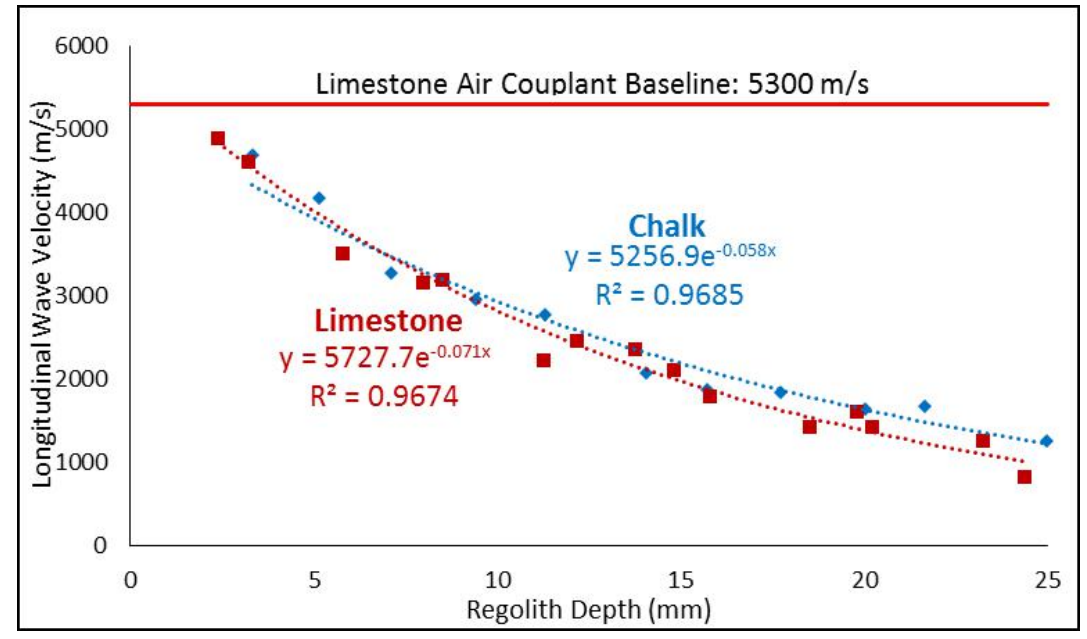

Figure 4.13: Regolith Depth vs. Longitudinal Wave Velocity for Dissimilar Regolith and Core Material. 


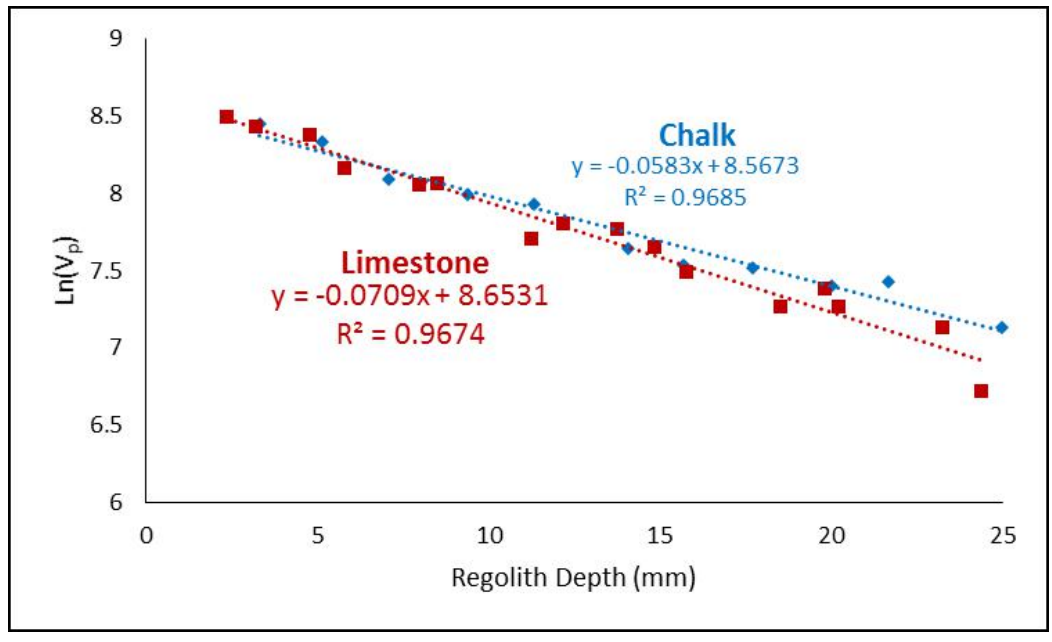

Figure 4.14: R5 Linearized Curves

\begin{tabular}{|lcc|}
\hline Significance Test & $\mathbf{P}(\mathbf{F})$ & Statistically Different \\
\hline Scatter & 0.2809 & No \\
Slope & 0.0226 & Yes \\
Intercept & 0.000 & Yes \\
\hline
\end{tabular}

Table 4.8: R5 Comparison Between Trendlines

\subsection{Surface Roughness}

\subsubsection{Isolating the Parameter}

The purpose of the first surface roughness series was to isolate the effect of surface roughness on wave velocity. Eight limestone samples were prepared with one rough surface parallel to a smooth end. After testing, the rough ends were removed and smoothed out and tested again. Gel couplant was also added. Figure 4.15 shows the results in box-plot form.

When the rough ends of the samples were removed and no gel couplant was added, the average wave velocity increased by about $700 \mathrm{~m} / \mathrm{s}$ from $4554 \mathrm{~m} / \mathrm{s}$ to $5253 \mathrm{~m} / \mathrm{s}$ or a $15 \%$ increase ; however, when gel couplant was added, an average increase of 200 $\mathrm{m} / \mathrm{s}$ from $5540 \mathrm{~m} / \mathrm{s}$ to $5743 \mathrm{~m} / \mathrm{s}$ occurred, which is about a $3.6 \%$ increase. Between rough samples, the addition of couplant increased wave velocity by nearly $22 \%$, but between smooth samples, the addition of couplant increased wave velocity by $9.3 \%$. The effect of surface roughness on signal attenuation is dependent on the amount of void space in between the surface of the sample and the transducer. The addition of coupling gel can fill in the void spaces and improve wave velocity. Without it, the 


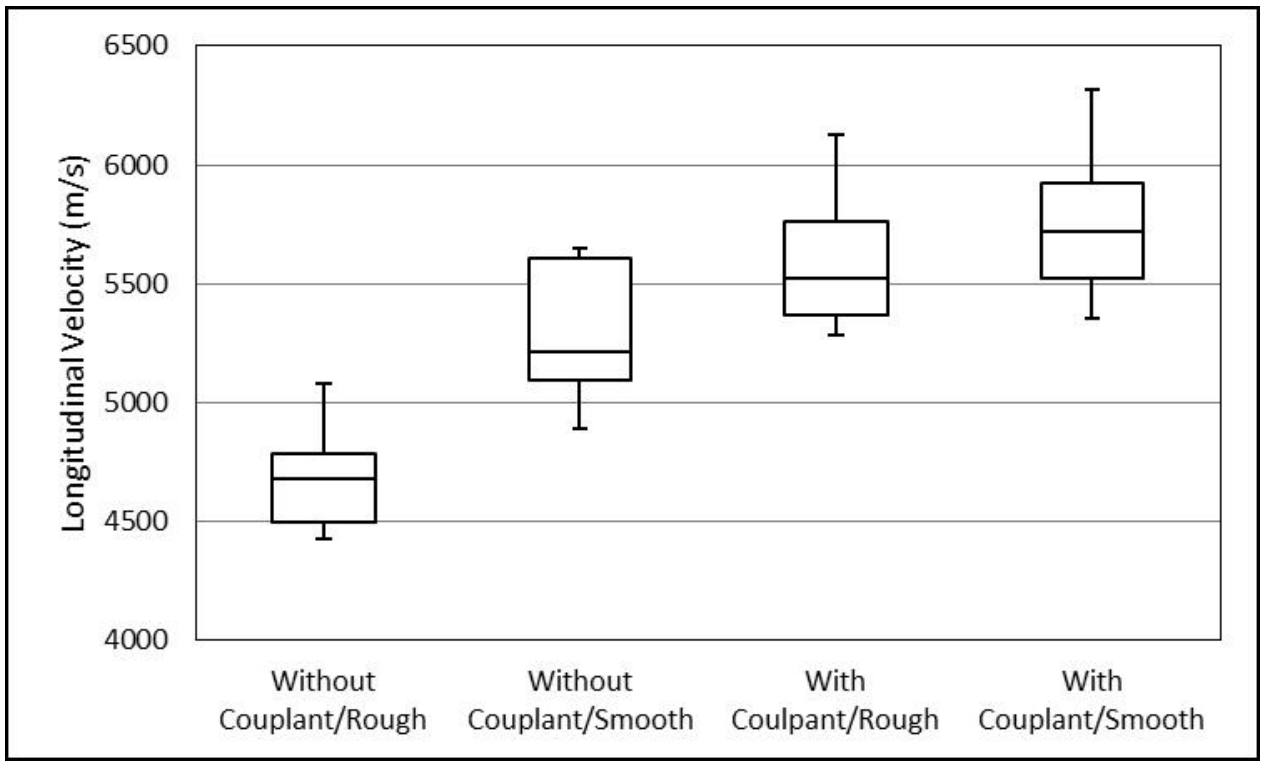

Figure 4.15: Comparison Between Smooth and Rough Samples With and Without Gel Couplant

signal attenuation is lost through the air and decreases the wave velocity to point where it is not indicative of the mechanical properties of the testing sample.

\subsubsection{Addition of Surface Regolith and Comparison to R4}

The second roughness test series attempted to establish whether or not the addition of regolith on the surface of a rough sample will produce similar results to a smooth sample with a regolith cover. The results indicate if surface regolith can act as a couplant since the regolith has a similar acoustic impedance to the core body. Since the 1000-2000 micron size class proved to be the best out of the three tested size classes in the fourth regolith series, it was used as the surface regolith. The results of the test were compared to the those gathered in R4 on a different but smooth limestone core. The exponential curves made from the data can be seen in Figures 4.16 . 


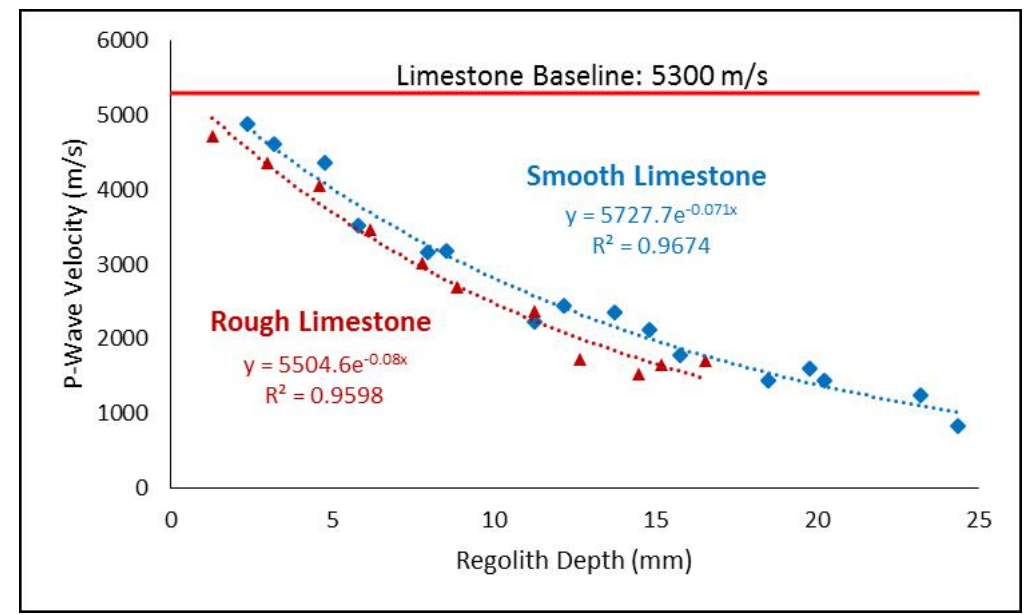

Figure 4.16: Regolith Depth vs. Longitudinal Wave Velocity for Smooth and Rough Samples

Figure 4.16 shows that the data stops at about $16.5 \mathrm{~mm}$ instead $25 \mathrm{~mm}$, possibly due to waves refracting when they make contact with the rough surface of the sample; however, the strong coefficients of determination and similar slopes indicate that regolith acts as a couplant to the surface of the rough sample. The regolith laying on top of the rough sample effectively makes a smooth surface because the particles fill in some of the void space between the sample and the transudcer, which produces a similar wave velocity trendline to a smooth core sample. Since two different limestone samples were used, comparisons between the corrected values were incompatible. Thus, a two-tailed t-test calculated if the corrected value from the rough limestone test was significantly different from the average velocity of the rough samples with gel couplant tests in the previous roughness series. Using a p-value of 0.05 , the t-test concluded that the corrected value was not significantly different from the results gathered in the previous roughness series and indicates that the corrected value is an accurate estimation of the baseline velocity.

\section{$4.4 \quad$ Ice Testing}

\subsubsection{Variance in Ice Concentration}

A final test series was conducted to determine if the UPV sensor can be used to detect changes ice content in icy regolith mixtures while varying regolith particle sizes and distributions. Icy regolith core samples were manufactured by mixing water and regolith to create a blend with a mass of 100 grams. Samples were then frozen overnight in a standard freezer, and UPV testing was conducted immediately after removal from the freezer. The particle size distribution of the regolith was constrained 
as an experimental parameter and no gel couplant was used since the liquid on top of the sample acts as a couplant. Figure 4.17 shows the results of the first test series.

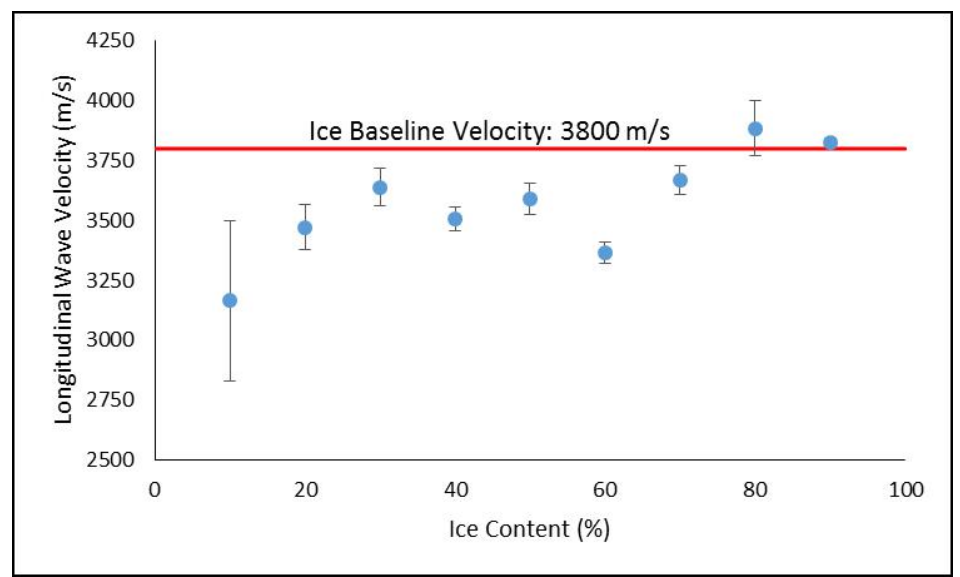

Figure 4.17: Regolith UPV Testing with a Monosized Regolith Mixed with Ice

As ice concentration steadily increases, the wave velocity increases as well until it hits the velocity baseline of ice at around $3800 \mathrm{~m} / \mathrm{s}$. Although the velocity decreases between 40 and $60 \%$ ice concentration, the wave velocity quickly increases to the baseline value of ice, which corroborates with conclusions from literature that state that wave velocity will increase with ice concentration. This test used only one particle size class whereas realistically, the regolith will be made of multiple size classes.

\subsubsection{Distribution of Regolith Sizes and Ice}

Three different distributions of regolith were mixed with ice at different concentrations to observe whether the same trend found previous the section would present itself if the distribution of particle sizes changed. The results from the nine samples can be seen in Figure 4.18

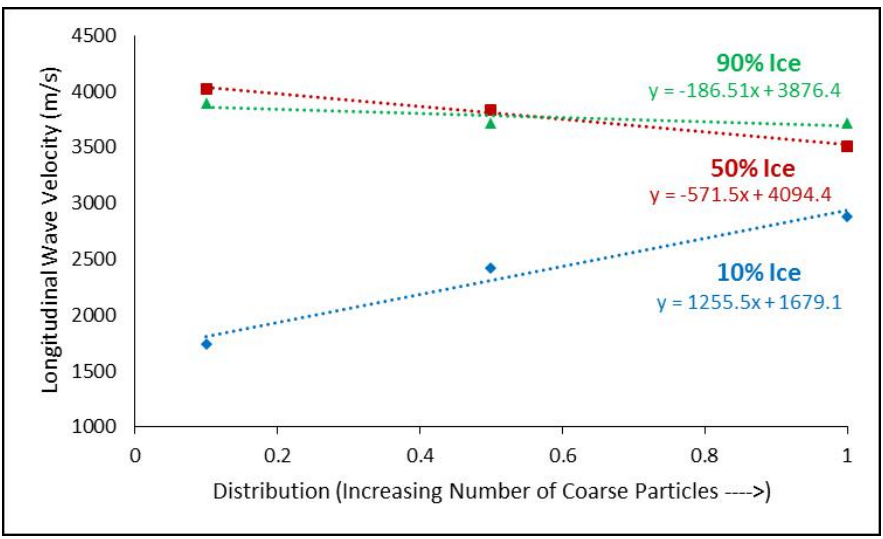

Figure 4.18: Regolith Distribution and Ice Testing 
As the distribution of particles increases, meaning a greater number of coarser particle sizes, three different trends appear. With $10 \%$ ice by mass, as the number of coarse particle sizes increase, wave velocity begins to increase and corroborates with findings from Section 4.2.3 that large particle sizes impact signal attenuation less than smaller particles. This is contested by the data from the $50 \%$ ice concentration dataset that has a slightly decreasing trendline. This trendline corroborates with data from the previous section that shows a slight decrease in wave velocity at $50 \%$ ice concentration. The decrease in wave velocity may be due to the even concentrations of ice and regolith, and yields a mixture that possesses the qualities of neither material. The $90 \%$ concentration trendline is consistent across all distributions at around the ice baseline velocity of $3800 \mathrm{~m} / \mathrm{s}$ since the mixture is almost a solid piece of ice. Together with the data from the previous section, the wave velocity is not just dependent on the distribution of particles, but mostly based on the mass of particles present.

\subsection{Discussion and Implications}

When analyzing the results of these tests, the data provides compelling evidence that a true value for $\mathrm{P}$-wave (longitudinal) velocity of a certain material can be estimated when performing in-situ UPV tests. Several UPV measurements at different lengths have to be taken in order to construct the signal attenuation curve as a wave passes through the body. The signal attenuation curve is based on the measured wave velocities, which can estimate the true value of the $\mathrm{P}$-wave velocity when the trendline is extrapolated to zero. Surface roughness can be mitigated by the addition of a gel couplant, but if it is not available then an in-situ replacement has to be found.

Regolith covering the surface of a rock mass can affect the signal attenuation of an ultrasonic wave even in shallow depths of a couple of centimeters. Not only does regolith depth affect the attenuation of the ultrasonic wave, but the particle sizes and distribution of the particle sizes will affect it as well; however, a precise amount and certain size distribution of regolith can provide a smooth surface on a rough sample for testing, while limiting the amount of signal attenuation. Coarse particle sizes and narrower distributions as well as a shallow regolith depth will limit the amount of signal attenuation. Finally, the reoglith has to be compacted to its maximum to limit the amount of void spaces. The following sections specify the influence and abatement strategy for each condition.

For a spacecraft testing in-situ, the UPV responses produced on different bodies will likely have site-specific correlations, similar to what is observed on Earth. The regolith particle sizes and their distributions as well as their chemical composition will also vary site to site. The regolith on one asteroid or on one site of the Moon will be different from most other sites, which means initial measurements may not be as 
precise as intended. Although, once a database of characterized rocks develops, the precision and accuracy of this method will improve as there will be existing data to serve as comparisons.

For ice characterization, UPV can detect variations in ice concentrations mixed with regolith particles; however, the non-linearity of the relationship means that wave velocity value can have two meanings on a curve. It does not mean that UPV is cannot be used to a characterize ice, it may be a secondary option if none are available. Additionally, different materials mixed with ice can have different responses as opposed to ones seen in this body of research.

\subsubsection{Sample Length}

Influence Transmission length was shown to be a significant influence on measured UPV. As the distance between the transducers increased, measured wave velocities decreased. At lengths approaching 6 meters, measured UPV was be reduced by up to $10 \%$ of the measured value for the cases using gel couplant and $23 \%$ for cases without gel couplant.

Abatement/Mitigation Take at least 4 measurements at different transmission lengths up to the point where the signal is no longer observable. Fit the data set with an exponential trendline. The coefficient of the exponential term (i.e. exponential intercept) is the estimate of true baseline UPV. Larger spacing between the measurements and more measurements produce more accurate predication, but even at 0.25 $\mathrm{m}$ spacing, the average error between the estimate and the measured value can be less than $0.2 \%$.

\subsubsection{Regolith Cover}

Influence Regolith cover was shown to influence both signal amplitude and measured velocity with an increasing amount of cover leading to decreases in both responses. The size of the regolith particles also influenced signal amplitude and velocity with finer particles leading to a greater decrease in wave velocity than larger particle sizes but larger particle sizes have a greater impact on signal amplitude. At depths exceeding $2.5 \mathrm{~cm}$, no signal was observed, and at depths of about $1.2 \mathrm{~cm}$, measured values were reduced by 53 - $63 \%$ compared to the baseline value.

Abatement/Mitigation Take at least three measurements at varying regolith depths up until a signal cannot be received. During each measurement, compact regolith as much as possible, and try to obtain values with a minimal regolith cover. 
Use a linear trendline to extrapolate to zero depth. If possible, note the particle size, and recall that sizes $<1 \mathrm{~mm}$ will induce some error. If performed properly, this procedure can reduce the error between the baseline and predicted value to less than $8 \%$. If gel couplant is available, the regoltih cover can removed and gel couplant can fill in the voids between the transducers and specimen.

\subsubsection{Sample Roughness}

Influence Sample roughness was shown to significantly affect measured wave velocity. A rough surface limits the amount of contact area between the transducer and the surface of the sample. Without the use of a gel couplant, the average wave velocity was $20.7 \%$ slower than velocities from samples prepared for laboratory testing (smooth surfaces and addition of coupling gel).

Abatement/Mitigation As long as the transducers are parallel to each other, the addition of a coupling gel will mitigate the effects of surface roughness. The coupling gel reduces the difference between measured wave velocities of the rough samples and ideal lab samples to within $200 \mathrm{~m} / \mathrm{s}(3.5 \%)$ on average. A regolith couplant can also be used to provide a smooth surface for testing and provide a corrected velocity using the abatement strategy in Section 4.5.2

\subsubsection{Gel Couplant}

\section{Abatement/Mitigation}

Use couplant if at all possible, but if not, then a small amount of surface regolith (less than $2.5 \mathrm{~cm}$ ) will provide a smooth surface for testing. If possible, the regolith needs to contain coarser particle sizes greater than $1 \mathrm{~mm}$ since wave velocities will decrease at a greater rate with finer particle sizes. Then follow the abatement strategy to obtain the exponential trendlines outlined in Section 4.5.2. 


\section{Chapter 5}

\section{Conclusions and Recommendations}

\subsection{Conclusions}

This work describes the development of a method for in-situ characterization of extraterrestrial rock masses using ultrasonic waves. While ultrasonic waves have been used as a conventional method for rock characterization for terrestrial uses, the standard laboratory settings do not take into account in-situ physical and environmental conditions. From the literature review and standard testing protocols, there are four conditions identified that require investigation: the length of the sample, the roughness of its surface, and the regolith that may be covering it. Due to the uncertainty of the effects of environmental conditions faced in space on commercially available coupling gel, the absence of a coupling gel normally used for efficient energy transfer between transducers and the rock sample has to be assessed as well. A secondary objective of characterizing ice mixed with a regolith sample was also investigated since water is critical for ISRU efforts. The conclusions relevant to original objectives are:

- Sample length, roughness, regolith cover, and the absence of gel couplant were identified and assessed as in-situ physical sample properties that affected UPV signal attenuation.

- Procedures for mitigating the effects of the properties were developed.

- The ability of UPV to characterize changes in ice content was partially assessed.

To assess the influence of sample length, three concrete beams were constructed in addition to multiple concrete cores. The cores provided a baseline $\mathrm{P}$-wave velocity for the concrete while measurements were taken with direct and semi-direct transmissions along the length of the six meter long beam with and without gel couplant. The results showed that the velocity at zero length from the regression lines can estimate the baseline velocity accurately with or without couplant, and commercially available 
transducers can reach the target length albeit with decreased accuracy especially without couplant. Further error analysis showed the maximum possible error was much larger when not using couplant and will require more data points to precisely estimate a wave velocity.

The regolith testing evaluated the influence of different parameter that comprise a regolith sample on signal attenuation. A Box-Behnken experimental design for four parameters was conducted: particle top size, particle size distribution, depth, and compaction. The results from the first series show that the depth of the regolith cover is the most dominant influence on signal attenuation through a sample while compaction, top size, and distribution had various effects on wave amplitude and velocity. The full factorial experimental design for the second series eliminated compaction as a variable by compacting samples to their maximum and a mass of regolith was used instead of a precise depth measurement. The results from the second series emphasize the importance of particle size, and display an exponential relationship between increasing particle depth and decreasing wave velocity; however, the importance of a changing particle size could not be isolated. The third and fourth series identified the effect of particle size by using individual size classes. The results show that larger particle sizes had less of an effect on signal attenuation than smaller classes meaning a more precise wave velocity estimation to a baseline value. The fifth series used a single size fraction of chalk particles and placed increasing amounts on top of the same limestone core used in previous testing to evaluate the effect of an different regolith material on signal attenuation. The trendline produced had a statistically different slope and intercept from the trendline produced with limestone regolith in the fourth series. The results show that a difference in regolith material from the rock mass beneath will provide inaccurate results.

The surface roughness testing attempted to isolate the effect of the surface texture on the sample and provide a method to mitigate it. Eight limestone samples with a single rough surface were tested with and without gel couplant. Once the testing was over, the rough surfaces were removed, and the samples were tested again with and without gel couplant. Without the addition of a gel couplant, the rough surfaces significantly affect wave velocity whereas couplant addition increases wave velocity to similar values attained with smooth surfaces and a gel couplant. Gel couplant can eliminate the effects of surface roughness as long as the transducers are aligned correctly; however, a substitute has to be found if gel couplant is not available. The second roughness series used one of the size classes of limestone regolith from the fourth regolith series as well as the same procedure to determine if similar attenuation curves can be produced. The attenuation curve produced is similar in terms of slope and scattering with the one created in the fourth regolith series. These similarities mean that the regolith particles fill in the void spaces between the transducer and 
sample to produce a flat testing surface, which distributes ultrasonic waves uniformly across the surface. A t-test concluded that the corrected value was not significantly different from the results gathered in the first roughness series and indicates that the corrected value is an accurate estimation of the baseline velocity.

To determine an estimation of the true P-wave velocity of an extraterrestrial rock mass, multiple UPV measurements have to be taken across the length of the sample to generate the signal attenuation curve. The empirically derived regression equation can extrapolated to zero depth, which will provide an estimation for the baseline velocity. The addition of surface regolith and roughness complicates the procedure. Both properties increase signal attenuation but can be mitigated by the removal of the regolith and the addition of coupling gel to the surface. The complications increase if coupling gel is not provided on the mission. The regolith can act as couplant and in small amounts, it can create a smooth surface on top of a rough sample and limit the amount of signal attenuation in order to estimate the true P-wave velocity. Gel couplant is strongly recommended to be a part of the UPV system, so the regolith cover can be simply removed.

To accomplish the secondary objective, different mixtures of ice and limestone particles were created to evaluate the ability of UPV to detect changes in ice content. The results show that UPV can detect variances in ice concentrations mixed with regolith particles; however, the non-linearity of the relationship means that measured wave velocity can have two meanings on a curve. It does not mean that UPV is cannot be used to characterize ice mixtures, but more research is needed to provide a final analysis.

\subsection{Recommendations/Future Work}

While this research has demonstrated a method for in-situ UPV testing, further work is required to determine additional uses for UPV as a tool for extraterrestrial exploration. The author of this thesis recommends the following areas for additional research may be continued:

1. Further work with UPV as means for extraterrestrial rock characterization is required by incorporating all three physical parameters into small and large scale samples. This body of research conduct tests evaluating three separate physical parameters, but the next step is to combine these parameters to validate the procedure suggested. Not only should variations in sample size, texture, and regolith coverings be present but also different sample shapes and sample internal structure. A rock is irregularly shaped and rarely provides parallel surfaces to work with and the internal structure of a sample can vary significantly between 
specimens, so the procedure suggested must be able to adapt in order to provide accurate velocity estimations. The new test series can also integrate robotics into the experimental design to develop additional procedures for remote and autonomous rock characterization.

2. The results from the ice characterization tests are incomplete and a more detailed investigation is warranted. This objective can be achieved by experimenting with different regolith materials that are characteristic of a regolith found on an extraterrestrial body. The mineralogy of the regolith, its particle particle sizes and distributions as well as the amount of pore space should vary in order to simulate more realistic conditions as those faced on the surface an extraterrestrial body.

3. Development of a method that incorporates the results from this thesis, Savage (2016) and Goodman (2016). The method will provide comprehensive strategies to mitigate environmental and physical properties as well as robotic issues when conducting UPV tests. 


\section{Bibliography}

Angel Abbud-Madrid, David Beaty, Dale Boucher, Ben Bussey, Richard Davis, Leslie Gertsch, Lindsay Hays, Julie Kleinhenz, Michael Meyer, Michael Moats, et al. Mars water in-situ resource utilization (ISRU) planning (M-WiP) study. 2016.

Seyed Hassan Amini, Rick Honaker, and Aaron Noble. Performance evaluation of a dense-medium cyclone using alternative silica-based media. Powder Technology, 297:392-400, 2016.

M Anand, Ian A Crawford, M Balat-Pichelin, S Abanades, W Van Westrenen, G Péraudeau, R Jaumann, and W Seboldt. A brief review of chemical and mineralogical resources on the moon and likely initial in situ resource utilization (ISRU) applications. Planetary and Space Science, 74(1):42-48, 2012.

ASTM Standard A388/ A388M-16a. Standard practice for ultrasonic examination of steel forgings.ASTM International, West Conshohocken, 2016. URL https: //doi.org/10.1520/A0388_A0388M-16A.

ASTM Standard D2845-08. Standard test method for laboratory determination of pulse velocities and ultrasonic elastic constants of rock. ASTM International, West Conshohocken, 2008. URL https://doi .org/10.1520/D2845-08.

ASTM Standard D6760-16. Standard test method for integrity testing of concrete deep foundations by ultrasonic crosshole testing. ASTM International, West Conshohocken, 2016. URL https://doi.org/10.1520\%2Fd6760.

Adnan Aydin. Upgraded ISRM Suggested Method for Determining Sound Velocity by Ultrasonic Pulse Transmission Technique, pages 95-99. Springer International Publishing, Cham, 2015. ISBN 978-3-319-07713-0. doi: 10.1007/978-3-319-07713-0_6. URL http://dx.doi.org/10.1007/978-3-319-07713-0_6.

Paula G Benavidez, Daniel D Durda, Brian L Enke, William F Bottke, David Nesvornỳ, Derek C Richardson, Erik Asphaug, and William J Merline. A comparison between rubble-pile and monolithic targets in impact simulations: Application to asteroid satellites and family size distributions. Icarus, 219(1):57-76, 2012. 
Jean-Pierre Bibring, Yves Langevin, John F Mustard, François Poulet, Raymond Arvidson, Aline Gendrin, Brigitte Gondet, Nicolas Mangold, P Pinet, F Forget, et al. Global mineralogical and aqueous mars history derived from omega/mars express data. Science, 312(5772):400-404, 2006.

E Blomme, D Bulcaen, and F Declercq. Air-coupled ultrasonic nde: experiments in the frequency range 750khz-2mhz. NDT \& e International, 35(7):417-426, 2002.

DT Britt, D Yeomans, K Housen, and G Consolmagno. Asteroid density, porosity, and structure. Asteroids III, 1987.

Benoit Carry. Density of asteroids. Planetary and Space Science, 73(1):98-118, 2012.

Martin Christ and Jun-Boum Park. Ultrasonic technique as tool for determining physical and mechanical properties of frozen soils. Cold Regions Science and Technology, 58(3):136-142, 2009.

Ebrahim Çobanoglu and Sefer Beran Çelik. Estimation of uniaxial compressive strength from point load strength, schmidt hardness and p-wave velocity. Bulletin of Engineering Geology and the Environment, 67(4):491-498, 2008.

Anthony Colaprete, Peter Schultz, Jennifer Heldmann, Diane Wooden, Mark Shirley, Kimberly Ennico, Brendan Hermalyn, William Marshall, Antonio Ricco, Richard C. Elphic, David Goldstein, Dustin Summy, Gwendolyn D. Bart, Erik Asphaug, Don Korycansky, David Landis, and Luke Sollitt. Detection of water in the lcross ejecta plume. Science, 330(6003):463-468, 2010. ISSN 0036-8075. doi: 10.1126/science. 1186986. URL http://science.sciencemag.org/content/330/6003/463.

FE DeMeo, CMOD Alexander, KJ Walsh, CR Chapman, and RP Binzel. The compositional structure of the asteroid belt. Asteroids $I V, 1: 13,2015$.

K Diamantis, E Gartzos, and G Migiros. Study on uniaxial compressive strength, point load strength index, dynamic and physical properties of serpentinites from central greece: test results and empirical relations. Engineering Geology, 108(3): 199-207, 2009.

Bethany L Ehlmann and Christopher S Edwards. Mineralogy of the martian surface. Annual Review of Earth and Planetary Sciences, 42:291-315, 2014.

DC Entwisle, PRN Hobbs, LD Jones, D Gunn, and MG Raines. The relationships between effective porosity, uniaxial compressive strength and sonic velocity of intact borrowdale volcanic group core samples from sellafield. Geotechnical \& Geological Engineering, 23(6):793-809, 2005. 
TH Gan, DA Hutchins, DR Billson, and DW Schindel. The use of broadband acoustic transducers and pulse-compression techniques for air-coupled ultrasonic imaging. Ultrasonics, 39(3):181-194, 2001.

Drew M Goodman. Concept and operation of robotic nondestructive testing systems for space exploration objectives. Master's thesis, West Virginia University, 2016.

WA Grandia and CM Fortunko. Nde applications of air-coupled ultrasonic transducers. In Ultrasonics Symposium, 1995. Proceedings., 1995 IEEE, volume 1, pages 697-709. IEEE, 1995.

Grant Heiken, David Vaniman, and Bevan M French. Lunar Sourcebook: A User's Guide to the Moon. CUP Archive, 1991.

WN Houston, JK Mitchell, and WD Carrier III. Lunar soil density and porosity. In Lunar and Planetary Science Conference Proceedings, volume 5, pages 2361-2364, 1974 .

Gokhan Inci, Nazli Yesiller, and Takaaki Kagawa. Experimental investigation of dynamic response of compacted clayey soils. ASTM International - Geotechnical Testing Journal, 26(2):125, 2003.

S Kahraman. Evaluation of simple methods for assessing the uniaxial compressive strength of rock. International Journal of Rock Mechanics and Mining Sciences, 38 (7):981-994, 2001.

S Kahraman. The effects of fracture roughness on p-wave velocity. Engineering Geology, 63(3):347-350, 2002.

Manoj Khandelwal. Correlating p-wave velocity with the physico-mechanical properties of different rocks. Pure and Applied Geophysics, 170(4):507-514, 2013.

Manoj Khandelwal and TN Singh. Correlating static properties of coal measures rocks with p-wave velocity. International Journal of Coal Geology, 79(1):55-60, 2009.

A. Kiliç and A. Teymen. Determination of mechanical properties of rocks using simple methods. Bulletin of Engineering Geology and the Environment, 67(2):237, 2008. ISSN 1435-9537. doi: 10.1007/s10064-008-0128-3. URL http://dx.doi .org/10 . $1007 /$ s 10064-008-0128-3.

Frederick V Lawrence Jr. Ultrasonic shear wave velocities in sand and clay. Technical report, DTIC Document, 1965. 
John S Lewis and Melinda L Hutson. Asteroidal resource opportunities suggested by meteorite data. Resources of Near-Earth Space, pages 523-542, 1993.

Daniel D Mazanek, Raymond G Merrill, John R Brophy, and Robert P Mueller. Asteroid redirect mission concept: a bold approach for utilizing space resources. Acta Astronautica, 117:163-171, 2015.

DM McCann and MC Forde. Review of ndt methods in the assessment of concrete and masonry structures. Ndt \& E International, 34(2):71-84, 2001.

Behnaz Minaeian and Kaveh Ahangari. Estimation of uniaxial compressive strength based on p-wave and schmidt hammer rebound using statistical method. Arabian Journal of Geosciences, 6(6):1925-1931, 2013. ISSN 1866-7538. doi: 10.1007/ s12517-011-0460-y. URL http://dx.doi.org/10.1007/s12517-011-0460-y.

DA Mishra and A Basu. Estimation of uniaxial compressive strength of rock materials by index tests using regression analysis and fuzzy inference system. Engineering Geology, 160:54-68, 2013.

ZA Moradian and M Behnia. Predicting the uniaxial compressive strength and static youngâs modulus of intact sedimentary rocks using the ultrasonic test. International Journal of Geomechanics, 9(1):14-19, 2009.

Peter B Nagy and Laszlo Adler. Surface roughness induced attenuation of reflected and transmitted ultrasonic waves. The Journal of the Acoustical Society of America, 82(1):193-197, 1987.

Peter B Nagy and James H Rose. Surface roughness and the ultrasonic detection of subsurface scatterers. Journal of Applied Physics, 73(2):566-580, 1993.

Yoshisuke Nakano and Robert Arnold. Acoustic properties of frozen ottawa sand. Water Resources Research, 9(1):178-184, 1973.

Yoshisuke Nakano, Randolph J Martin, and Martin Smith. Ultrasonic velocities of the dilatational and shear waves in frozen soils. Water Resources Research, 8(4): 1024-1030, 1972.

TJ Napier-Munn. Statistical Methods for Mineral Engineers-How to Design Experiments and Analyse Data, volume 5. Julius Kruttschnitt Mineral Research Centre, 2014.

Marcia L Nelson, Daniel T Britt, and Larry A Lebofsky. Review of asteroid compositions. Resources of Near-Earth Space, pages 493-522, 1993. 
Sung Jun Oh, Yung C Shin, and ES Furgason. Surface roughness evaluation via ultrasonic scanning. IEEE Transactions on Ultrasonics, Ferroelectrics, and Frequency Control, 41(6):863-871, 1994.

James Papike, Steven Simon, and Lawrence Taylor. Lunar minerals. Lunar Sourcebook, pages 121-181, 1991.

Jung-Hee Park, Jong-Sub Lee, Seung-Seo Hong, and YoungSeok Kim. Properties of elastic waves in sand-silt mixtures due to freezing. In Mechanical Properties of Frozen Soil. ASTM International, 2013.

C. M. Pieters, J. N. Goswami, R. N. Clark, M. Annadurai, J. Boardman, B. Buratti, J.-P. Combe, M. D. Dyar, R. Green, J. W. Head, C. Hibbitts, M. Hicks, P. Isaacson, R. Klima, G. Kramer, S. Kumar, E. Livo, S. Lundeen, E. Malaret, T. McCord, J. Mustard, J. Nettles, N. Petro, C. Runyon, M. Staid, J. Sunshine, L. A. Taylor, S. Tompkins, and P. Varanasi. Character and spatial distribution of oh/h2o on the surface of the moon seen by $\mathrm{m} 3$ on chandrayaan-1. Science, 326(5952):568572, 2009. ISSN 0036-8075. doi: 10.1126/science.1178658. URL http://science. sciencemag.org/content/326/5952/568.

Kara A Savage. Nondestructive methods to characterize rock mechanical properties at low-temperature: Applications for asteroid capture technologies. Master's thesis, West Virginia University, 2016.

Daniel J Scheeres, Christine M Hartzell, Paul Sanchez, and Micheal Swift. Scaling forces to asteroid surfaces: The role of cohesion. Icarus, 210(2):968-984, 2010.

PK Sharma and TN Singh. A correlation between p-wave velocity, impact strength index, slake durability index and uniaxial compressive strength. Bulletin of Engineering Geology and the Environment, 67(1):17-22, 2008.

Desislava Z Slavova, David M Weidinger, Adam F Sevi, and Louis Ge. Evaluation of compacted silt characteristics by ultrasonic pulse velocity testing. In GeoFlorida 2010: Advances in Analysis, Modeling \& Design, pages 1284-1293. 2010.

RW Stephenson. Ultrasonic testing for determining dynamic soil moduli. In Dynamic Geotechnical Testing. ASTM International, 1978.

Raymond Sullivan, R Anderson, J Biesiadecki, T Bond, and H Stewart. Cohesions, friction angles, and other physical properties of martian regolith from mars exploration rover wheel trenches and wheel scuffs. Journal of Geophysical Research: Planets, 116(E2), 2011. 
David J Tholen. Asteroid taxonomic classifications. In Asteroids II, pages 1139-1150, 1989.

PC Thomas, J Wm Parker, LA McFadden, Cc T Russell, SA Stern, MV Sykes, and EF Young. Differentiation of the asteroid ceres as revealed by its shape. Nature, 437(7056):224-226, 2005.

A Timur. Velocity of compressional waves in porous media at permafrost temperatures. Geophysics, 33(4):584-595, 1968.

A Tugrul and IH Zarif. Correlation of mineralogical and textural characteristics with engineering properties of selected granitic rocks from turkey. Engineering Geology, 51(4):303-317, 1999.

DT Vaniman, DL Bish, DW Ming, TF Bristow, RV Morris, DF Blake, SJ Chipera, SM Morrison, AH Treiman, EB Rampe, et al. Mineralogy of a mudstone at yellowknife bay, gale crater, mars. Science, 343(6169):1243480, 2014.

Da-yan Wang, Yuan-lin Zhu, Wei Ma, and Yong-hong Niu. Application of ultrasonic technology for physical-mechanical properties of frozen soils. Cold Regions Science and Technology, 44(1):12-19, 2006.

David M Weidinger, Louis Ge, and Richard W Stephenson. Ultrasonic pulse velocity tests on compacted soil. In Characterization, Modeling, and Performance of Geomaterials: Selected Papers From the 2009 GeoHunan International Conference, pages 150-155, 2009.

Sarah L Wilkison, Mark S Robinson, Peter C Thomas, Joseph Veverka, Timothy J McCoy, Scott L Murchie, Louise M Prockter, and Donald K Yeomans. An estimate of eros's porosity and implications for internal structure. Icarus, 155(1):94-103, 2002 .

David R. Williams. Asteroids, 2015. URL Nssdc.gsfc.nasa.gov/planetary/text/ asteroids.txt.

Barry A Wills and Tim Napier-Munn. Wills' Mineral Processing Technology: An Introduction to the Practical Aspects of Ore Treatment and Mineral Recovery. Butterworth-Heinemann, 2015.

Nazli Yesiller, Gokhan Inci, and Carol J Miller. Ultrasonic testing for compacted clayey soils. In Advances in Unsaturated Geotechnics, pages 54-68. 2000.

Robert W Zimmerman and Michael S King. The effect of the extent of freezing on seismic velocities in unconsolidated permafrost. Geophysics, 51(6):1285-1290, 1986. 